\title{
BIKE-SHARING USAGE: MINING ON THE TRIP DATA OF BIKE-SHARING USERS
}


Graduation committee:

\section{Chairman/secretary}

Prof.dr.ir. A. Veldkamp University of Twente

\section{Supervisor}

Prof.dr.ir. M.F.A.M. van Maarseveen University of Twente

\section{Co-supervisors}

Dr. T. Thomas

University of Twente

\section{Members}

Prof.dr. M.J. Kraak

Prof.dr.ir. E.C. van Berkum

University of Twente

Prof.dr. Z. Huang

Prof.dr. K.J. Krizek

Dr. M.H.P. Zuidgeest

University of Twente

Shenzhen University, China

University of Colorado, Boulder,

USA

University of Cape Town, South Africa

ITC dissertation number 306

ITC, P.O. Box 217, 7500 AE Enschede, The Netherlands

ISBN 978-90-365-4394-1

DOI 10.3990/1.9789036543941

Cover designed by

Printed by ITC Printing Department

Copyright (c) 2017 by Ying Zhang

1U FACULTY OF GEO-INFORMATION SCIENCE AND EARTH OBSERVATION 


\title{
BIKE-SHARING USAGE: MINING ON THE TRIP DATA OF BIKE-SHARING USERS
}

\author{
DISSERTATION \\ to obtain \\ the degree of doctor at the University of Twente, \\ on the authority of the rector magnificus, \\ prof.dr. T.T.M. Palstra, \\ on account of the decision of the graduation committee, \\ to be publicly defended \\ on Wednesday 20 September 2017 at 14.45 hrs
}

by

Ying Zhang

born on 13 July 1987

in Xinyang, China. 
This thesis has been approved by

Prof. dr. M.F.A.M. van Maarseveen, supervisor

Dr. T. Thomas, co-supervisor 


\section{Acknowledgements}

Doing this PhD was a snap decision. The motivation behind this decision was that I wanted to have a special experience in my life. After five years, I have to say that I have reaped much more than what I had expected and this thesis. I do thank myself for being brave enough to choose this journey five years ago. This PhD research has been a challenging and enjoyable journey over the past five years. Without the support of the following people, this thesis could not be completed.

My first gratitude goes to my promoter Prof. M.F.A.M. van Maarseveen. I thank him for guiding me toward this challenging research. I appreciate the freedom he gave me to explore on my own, and his inspiration when we were discussing. His critical comments and constructive advice on my work have dramatically improved the quality of my research. Working with him, I learned the ways of thinking critically and defending my idea, and the ways of shaping my idea and telling a good story. I sincerely thank him for his support and encouragement during the past five years, and translating the summary of the thesis into Dutch.

Special thanks go to my daily supervisor, Dr. Tom Thomas. I was fortunate in having him as my daily supervisor. I sincerely appreciate his guidance and support in shaping ideas, improving analysis, and writing articles. From him, I learned how to explore the big data, which are very important to my research. His critical thinking and strong statistical background helped me improving the quality of work and enhancing my research skills. His words of encouragement gave me strength to go forward when I was in frustration. I thank him for always being there to give me advice.

I would express my gratitude to Mark Brussel for his supervision. I sincerely thank him for his continuous support and critical comments to my work, and offering advices in every stage of my research. I would also like to thank Dr. Mark Zuidgeest, who was my daily supervisor at the first year of my PhD study. Although five years have passed, I still remember how he helped me to start this PhD program. I sincerely appreciate his guidance and support in writing the research proposal.

I am deeply indebted to Prof. Huang Zhengdong, who paved the way for me to obtain the data. I appreciate all his help, support, and encouragement at every stage of my life. Special thanks to Mr. Zuo Zhijun, Mr. Zhang Cheng and Mr. Zhong Zhiyuan, who are affiliated with the Transport Department of the Urban Planning and Design Institute of Zhongshan (China). I thank them for collecting and sharing the data and the valuable help during the fieldwork. 
I would also like to express my gratitude to Loes Colenbrander for being kind and helpful to me during the past five years, as well as formatting the thesis. Special thanks go to F.H.M. van den Bosch for the technical assistance in data processing, and Petra Web for the kind help in scheduling meeting and answering my inquiries in the past five years. Thanks go to other colleagues in PGM department for their support and kindness.

I express my sincere gratitude to Fangyuan Yu for her company and support. I thank her for always being with me and helping me out when I was in frustration and in trouble. We had lots of fun: I enjoyed every journey with her, our day trip and brunch time on weekends, and so on. These wonderful moments do have colored my life. Special thanks to Qiuju for company and support at the first year of my PhD study. I thank her for all her help in both academic field and everyday life. Many thanks go to my other friends in ITC for sharing joys and life and their encouragement, they are: Sonia, Yiwen, Zhihui, Linlin, Oliver, Mathew, and Xi. I also thank my friends in China for always being on my side. Thank Xin Meng and Ruqin Yang for their long-term support. Special thanks to Xin Meng for helping me with front and back cover design.

I would like to thank the China Scholarship Council (CSC) and the Faculty ITC of University of Twente for the financial support.

Finally, I owe my deepest gratitude to my parents and grandparents for their unconditional love and support all the time. I have been fortunate to grow up in a family where I have the greatest freedom to explore the life as I wish, without any restrictions and pressures. Lastly, I would like to thank my husband, Peng Tao, for his unconditional love and company during the past ten years, for his encouragement and support when I was in frustration, and for his technical assistance in programming. I dedicate this dissertation to my family. 


\section{Table of Contents}

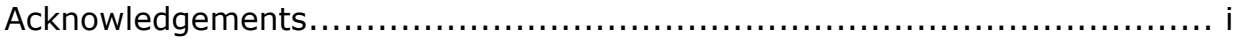

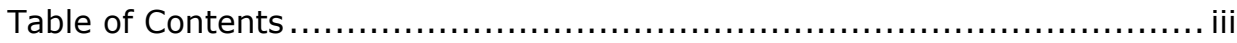

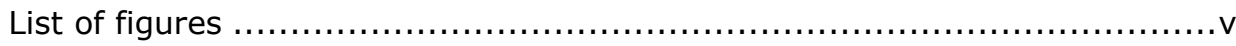

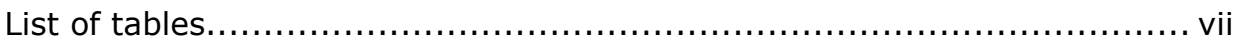

Chapter 1- Introduction ...........................................................

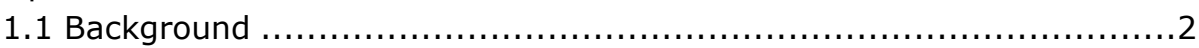

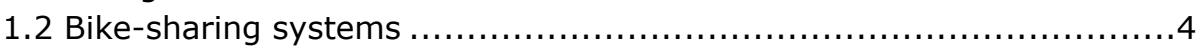

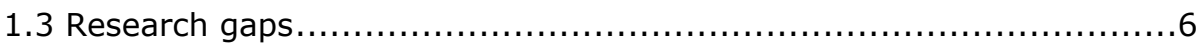

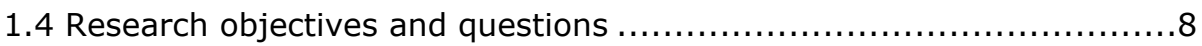

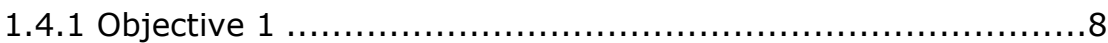

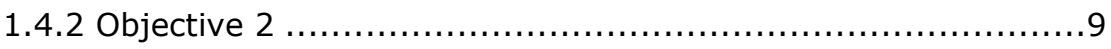

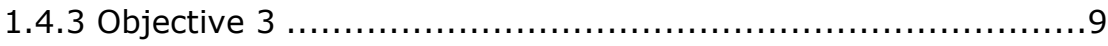

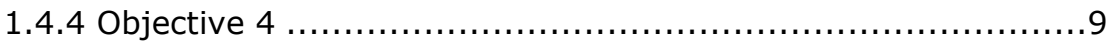

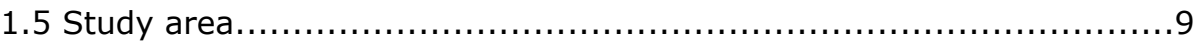

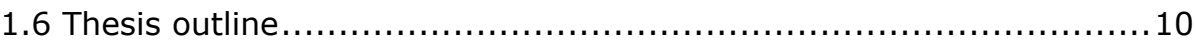

Chapter 2 - The characteristics of bike-sharing usage .......................... 13

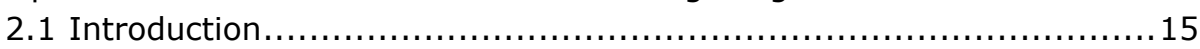

2.2 Research context ......................................................... 17

2.2.1 Study context .................................................. 17

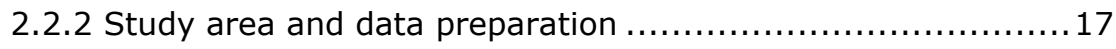

2.2.3 Characteristics in bicycle use: the user perspective ............. 18

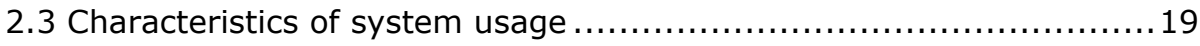

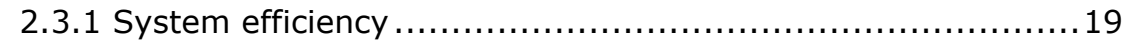

2.3.2 Trip characteristics ................................................. 21

2.3.3 Bicycle activity patterns at station level ........................ 22

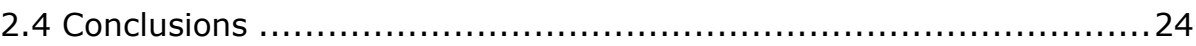

Chapter 3 - The impact of built environment factors on the bike

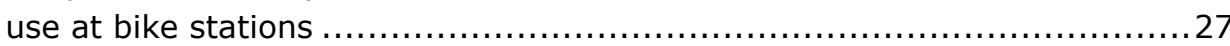

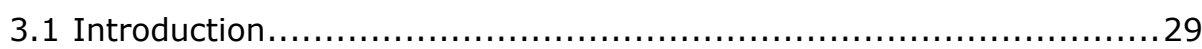

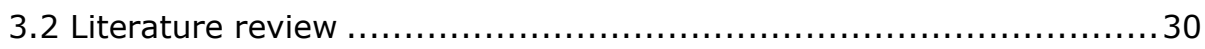

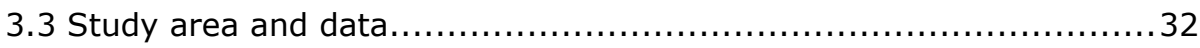

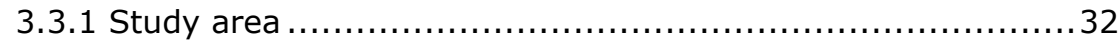

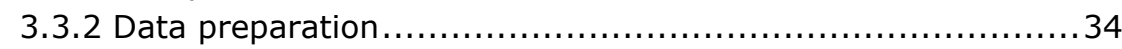

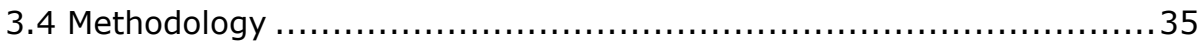

3.4.1 Selection and generation of dependent and explanatory

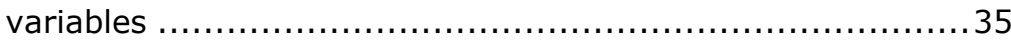

3.4.2 Multiple linear regression models.................................. 39

3.5 Analysis and results ....................................................... 41

3.5.1 Model results...................................................... 41

3.5.2 Model validation.................................................. 45

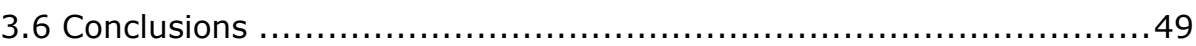

Chapter 4 - The dynamics of the bike-sharing system..........................5 53 


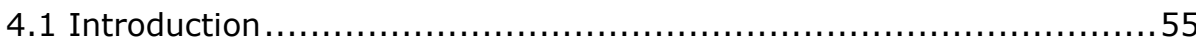

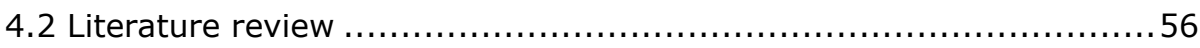

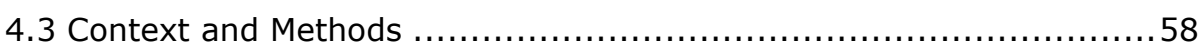

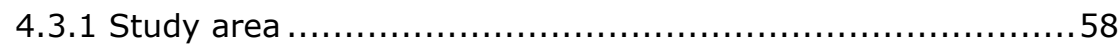

4.3.2 Zhongshan's bicycle-sharing system and data preparation ....59

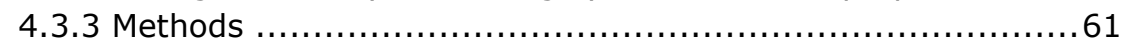

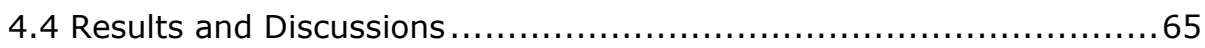

4.4.1 Aggregate use of the system before and after system expansion ........................................................... 65

4.4.2 Spatial distribution of demand before and after system

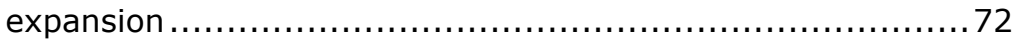

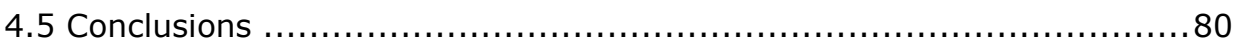

Chapter 5 - The travel behaviour of bike-sharing users ......................... 83

5.1 Introduction .............................................................. 85

5.2 State of the art researches in travel characteristics of bike-sharing

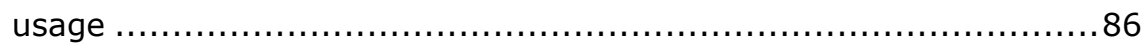

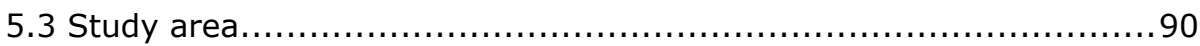

5.3.1 Transport condition in Zhongshan, China ........................ 90

5.3.2 Zhongshan's bike-sharing system ............................. 91

5.4 Data and Methods............................................................ 92

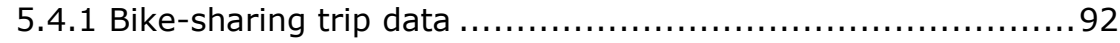

5.4 .2 Research design................................................. 93

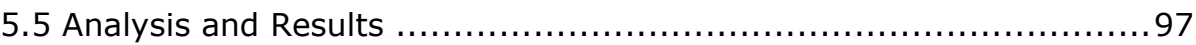

5.5.1 Types of trips, trip chains, and transition activities ..............97

5.5.2 Patterns of bike-sharing usage ................................. 100

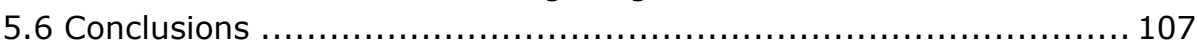

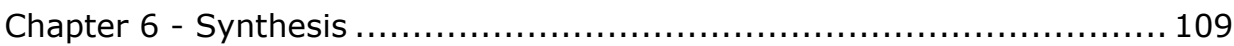

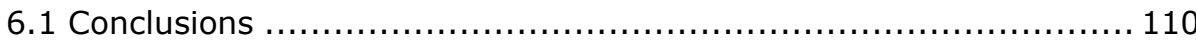

6.1.1 Objective 1: To examine the overall characteristics of bike-sharing usage in terms of system efficiency, trip characteristics, and station activity patterns............... 110

6.1.2 Objective 2: To examine the impact of built environment factors on the usage of public bikes at stations................ 111

6.1.3 Objective 3: To examine the dynamics of the bike-sharing system.

6.1.4 Objective 4: To examine the travel behaviour of bike-sharing users. ............................................... 114

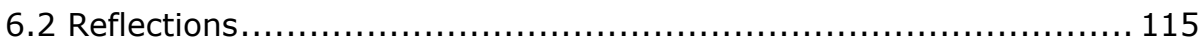

6.3 Recommendations for future research............................... 116

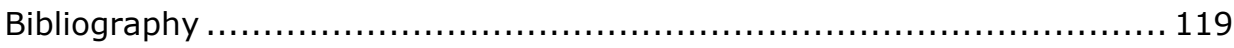

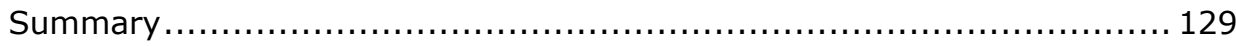

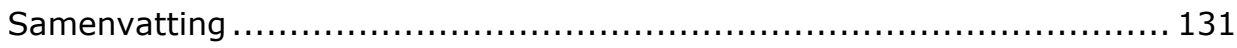

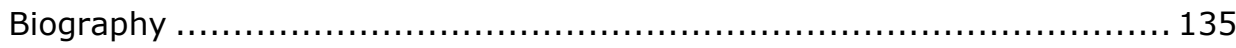

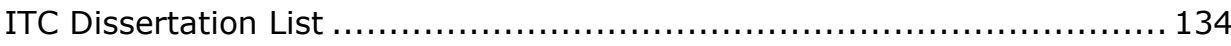

iv 


\section{List of figures}

Figure 1 The role of a bike-sharing system in urban transport. .................

Figure 2 The bike-sharing system in Nice, France. (Photography by author)..4

Figure 3 City Background. ............................................... 18

Figure 4 The division of six spatial zones with (A) population density and (B)

bike stations in the urban area................................... 19

Figure 5 Distribution functions and averages of trip durations. ................. 21

Figure 6 Weekday patterns of bicycle activity at stations...................... 23

Figure 7 Spatial distribution of station clusters............................. 24

Figure 8 Background of study area........................................... 34

Figure 9 Predicted $(Y)$ versus observed $(X)$ values at each station. ..........47

Figure 10 Unstandardized residual versus each significant independent variable at each station (Model $\ln [\mathrm{D} / \mathrm{S}]$ of weekdays). .............48

Figure 11 Spatial distribution of outliers and non-outlier (bike stations).....49

Figure 12 Study area. ................................................... 59

Figure 13 The spatial distribution of bike stations and capacities in the study

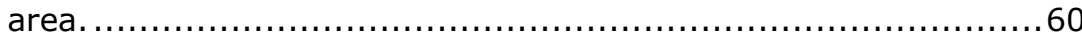

Figure $14 \mathrm{~A}$ diagram of computing the weight of each cell. ....................64

Figure 15 Comparisons of hourly demand during morning peak and evening peak hours at stations. ..........................................69

Figure 16 The spatial distribution of demand by all users on weekdays. Demand represents the number of trips per day. ..................73

Figure 17 Comparisons of daily demand by one group and another group at stations (weekdays) . ......................................... 74

Figure 18 Getis-Ord Gi* statistic of the spatial distribution of demand by each user group (weekdays) . ....................................... 75

Figure 19 The spatial distribution of D/S by all users on weekdays. The D/S represents the ratio of trips/day to the number of parking slots. .. 76

Figure 20 Differences in spatial demand by all users between weekdays and weekends. The "NDemand" represents the difference in normalized demand between weekdays and weekends.

Figure 21 Differences in spatial demand between user groups of before and after the system expansion (weekdays) ........................ 79

Figure 22 Differences in spatial demand between new users and steady users

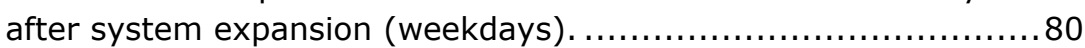

Figure 23 City Background............................................. 90

Figure 24 Modal split of commuting trips in TAZs. ......................... 91

Figure 25 (A)The spatial distribution of bike stations and (B) station capacity

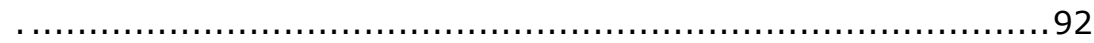

Figure 26 Bike-sharing trips originated from and arrived at the station.......93

Figure 27 Origin-Destination bicycle flows during morning peak and evening peak hours of weekdays....................................... 94

Figure 28 Dominant land use types of bike stations. ..................... 96 
Figure 29 The network distance between station $\mathrm{O}_{1}$ and station $\mathrm{D}_{\mathrm{F}}$ of nonloop trip chains on weekdays........................................ 100

Figure 30 Distribution of the network distance between transfer stations Dp and Os................................................................. 100

Figure 31 O-D matrix of individual trips (Type I ) on weekdays................ 102

Figure 32 O-D matrix of individual trips (Type II) on weekdays................ 102

Figure 33 O-D matrix of trip chains (Type II -A) on weekdays.................. 104

Figure 34 O-D matrix of trip chains (Type II -B) on weekdays. ................. 104

Figure 35 Network distance between the start and end stations of non-loop trip chains (7-9 a.m. 5-7 p.m.). .................................. 105

Figure 36 Transition matrix of bike-sharing usage (Type II -C) on weekdays. 106

Figure 37 Transition matrix of bike-sharing usage (Type II -D) on weekdays. 106 


\section{List of tables}

Table 1 Generations of bike-sharing programs.................................. 6

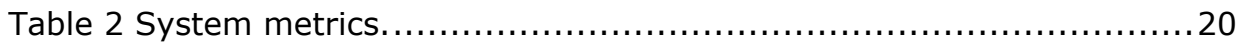

Table 3 Daily use of the system on weekdays...................................... 20

Table 4 O-D matrix of public bike trips (Weekdays)............................. 21

Table 5 Descriptive statistics of explanatory variables and daily and hourly

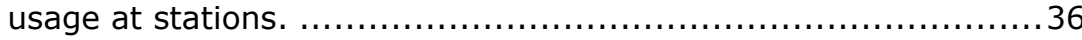

Table 6 Regression coefficients for estimated models -- dependent variable Ln[D] of weekdays, weekends, and holidays. .........................4 43

Table 7 Regression coefficients for estimated models -- dependent variable $\mathrm{Ln}[\mathrm{D} / \mathrm{S}]$ of weekdays, weekends, and holidays.

Table 8 Regression coefficients for estimated models -- dependent variable $\operatorname{Ln}[D]$ of MP, EP, and Off-peak. ......................................44

Table 9 Regression coefficients for estimated models -- dependent variable $\operatorname{Ln}[D / S]$ of MP, EP, and Off-peak. ....................................4 44

Table 10 The aggregate use of the system by all users in March 2012, March2013, and March 2014.......................................67

Table 11 The aggregate use of the system by steady users, former users, and new users on weekdays. ............................................. 70

Table 12 The aggregate use of the system by steady users, former users, and new users on weekends. ............................................. 71

Table 13 Definition and explanations of the terms used in this study..........95

Table 14 Types of individual bike-sharing trips (weekdays).....................98

Table 15 Types of transition activities and trip chains (weekdays). ...........99 
Chapter 1

Introduction 


\subsection{Background}

With the rapid development of motorization and urbanization, mobility and accessibility in cities are declining rapidly in the whole world (Gakenheimer, 1999; Sudhakara Reddy and Balachandra, 2012). Especially in fast growing cities of Asian countries, private car traffic has increased significantly, due to economic growth and the accompanied increase in car ownership and the transport policy focus on highway expansion as a solution to transport problems (Barter et al., 2003; Han, 2010). By now, there are various transport issues related to urban mobility: recurrent congestion, deterioration of urban accessibility, insufficient supply of (alternative) transport facilities, a mode shift from public to private transport, and so on (da Silva et al., 2008; de Vasconcellos, 2005; Gakenheimer, 1999; Sudhakara Reddy and Balachandra, 2012). Moreover, the increasing use of private transport brings about negative externalities, like traffic accidents, traffic congestion and air pollution (de Vasconcellos, 2005; EuropeanCommission, 2007).

Sustainable transport refers to any type of transport that is fuel-efficient, space-saving and encourages a healthy lifestyle. It calls for a balance between travel demand and resources needed by present and future generations (Han, 2010; Richardson, 2005). Particularly, non-motorized transport modes are considered vital determinants of sustainable transport (Rietveld and Daniel, 2004), with the bicycle being increasingly associated with benefits in terms of health, environment, society and economy (Jensen et al., 2010; Massink et al., 2011; Pucher et al., 2010; Vandenbulcke et al., 2011; Wardman et al., 2007).

Multimodal trips of bike with public transport contribute to more sustainable transport and the number of these trips has grown over the past decades (Keijer and Rietveld 2000; Martens, 2007). Hine and Scott (2000) indicated that integrated use of bicycle and public transport can make the transition and make public transport more attractive. As a feeder mode, the bicycle is faster than walking and more flexible than other public transport modes, and bicycle use in access/egress trips can substantially reduce the door-to-door travel time (Grotenhuis et al., 2007; Keijer and Rietveld 2000; Martens, 2007). However, given the growing consensus on the benefits of cycling, the important question for researchers and policy makers is how to stimulate cycling (Nkurunziza et al., 2012; Pucher et al., 2010).

Recently, bike sharing systems have been launched as an innovative and green mode of public transport to provide public access to rent and return public bikes at a number of bike stations across the urban area. Such systems not only act as a unimodal system, but also contribute to bridging the gap in urban transport systems to solve the "last mile" problem (Liu et al., 2012). Growing concerns about fast motorization and urban pollution, have led to increasing interests in bike-sharing programs across the world, from Europe to America, 
Asia, and Australia (Shaheen et al., 2010). Evidence has shown that cycling for both utilitarian and recreational purposes has increased in some cities after running bike-sharing programs (Fishman et al., 2013; O'Brien et al., 2014). Since 2016 a total of 1090 bike-sharing systems (BSS) are running in 60 countries worldwide, and many more will take off in the near future (Meddin and DeMaio, 2016).

The development of bike-sharing systems has experienced four generations (DeMaio, 2008). The latest generation of bike-sharing systems offers opportunities to access the operational database (e.g. station-based data or trip-level data), which enables new research possibilities. Yet very little scientific research has been reported that analyses the actual use of bikesharing systems employing the usage information embedded in the operational database. This database contains useful information on users' behaviour and system usage, which can be beneficial for the planning and implementation of improvement or expansion of existing systems, the design and adoption of new systems, as well as the operational management and maintenance. For instance, analysing the database can provide information developments in bike-sharing demand, the most suitable locations for bike-stations, or the necessary capacity of a bike-station. Moreover, it provides information on travel patterns and thereby contributes to a full understanding of the role of the bike-sharing system within the urban transport system (

Figure 1). This role might be dependent on local context: urban size, form and topology as well as the characteristics of the urban transport system.

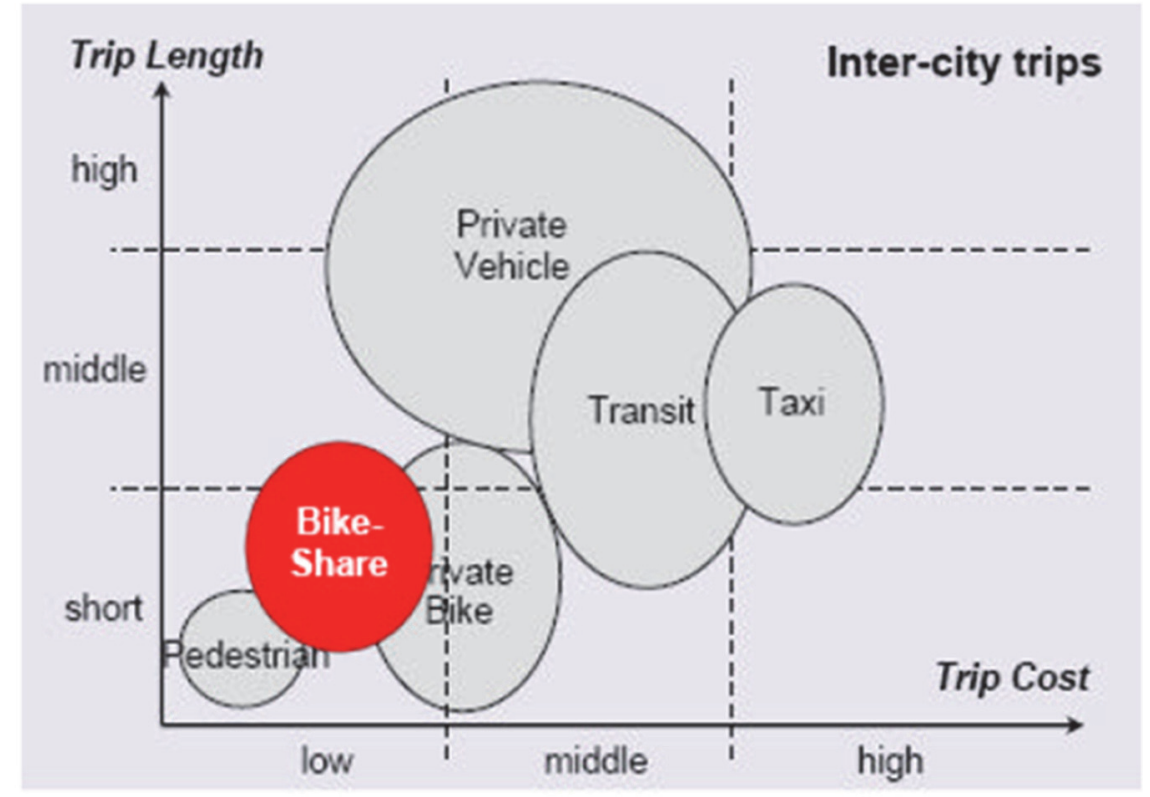

Source: CSD, 2011

Figure 1 The role of a bike-sharing system in urban transport. 
To contribute to a better understanding of the actual use and performance of bike-sharing systems, this research aims to develop methods and approaches to extract information from an operational database with respect to trip characteristics of bike-sharing users. This research is conducted for a bikesharing system in the city of Zhongshan, China. In particular in this country there is a huge interest in developing bike-sharing programs, as many new bike-sharing systems have been or are being launched in China. Yet most studies have been conducted in western cities, and only few researchers paid attention to Chinese bike-sharing systems (Fishman, 2016). Chinese cities exhibit different urban features than western cities, which makes an analysis of Chinese bike-sharing systems relevant and useful.

\subsection{Bike-sharing systems}

Bike-sharing systems are innovative programs of providing rental or free bicycles in inner urban areas, which differ from traditional mostly leisureoriented bicycle rental services. Such systems provide residents (or tourists) with a fast and easy access to public bicycles that can be used for both daily mobility and one way use (Bührmann, 2008). A bike-sharing system (Figure 2) consists of public bicycles that can be picked up and dropped off at a number of fixed bike stations across the urban area. Next to public bicycles and docking stations it has a service terminal for system access and user registration (CSD, 2011; TransportCanada, 2009). Public bicycles are available to the general public for free or against a small fee, and can be used in a unimodal fashion or as feeder mode to other transportation systems (public bus, metro, etc.) (Midgley, 2009). The objectives of bike-sharing programs are to increase bicycle use for short distance trips to improve the accessibility of other public transportation systems for trips over longer distances, as well as to reduce environmental impacts of current transport activities (DeMaio, 2009; Kaltenbrunner et al., 2010; Lin and Yang, 2011).
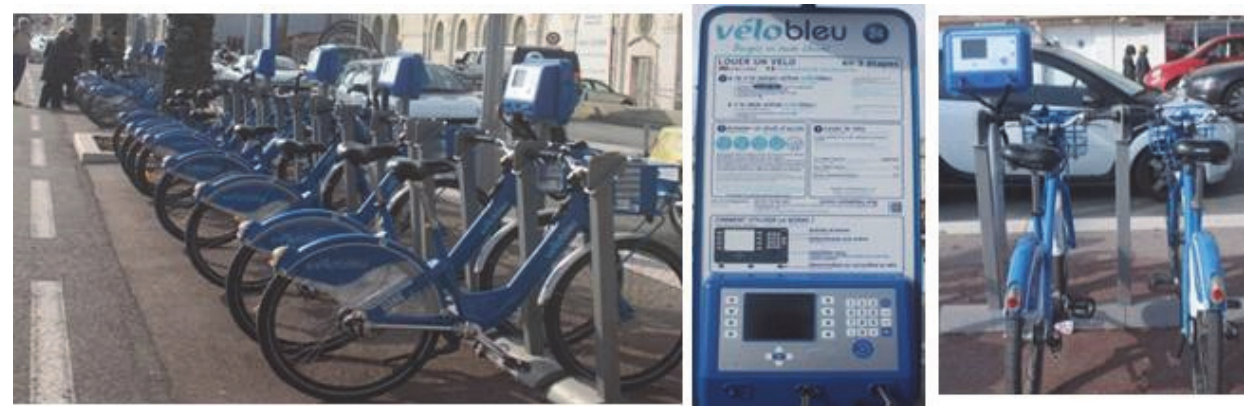

(a)Bike station; (b) IT Service terminal ; and (c) Parking slots.

Figure 2 The bike-sharing system in Nice, France. (Photography by author)

Bike-sharing systems primarily originated in Europe, but now it has been spread around Americas and Asia. By now, around 1090 bike-sharing systems 
are operating in 60 worldwide cities worldwide (Meddin and DeMaio, 2016). In retrospect to the evolution of public bike systems, they can be categorized into four generations (Table 1): white bikes, coin-deposit systems, information technology based systems and demand-responsive and multimodal systems (DeMaio, 2009; Shaheen et al., 2010). The 1st generation began in 1964 in Amsterdam called the White Bike Plan which at that time was seen as the solution to urban traffic problems in those days. The operators gathered a handful of bicycles that were painted white, left permanently unlocked and distributed across the inner city of Amsterdam for the public to use freely. However, this program didn't go as planned since the bikes were stolen or damaged, so that this system collapsed soon after the start. Nearly 30 years later, in 1995, the 2nd generation started operation in Copenhagen called Bycyklen, with many improvements in contrast to the previous generation. This bike sharing system was designed in such a way that users could pick up and return bikes at specific locations throughout the central city areas with a coin deposit. Despite the fact that 2 nd generation was more formalized than the previous generation with stations and a professional organization to operate the program, it still experienced the theft and damage of bicycles due to the anonymous use of customers. By contrast with the first two generations, the 3rd generation was smartened with a variety of technological improvements including electronically locking racks or bike locks, telecommunication systems, smartcards, mobile phone access and on-board computers. The 4th generation systems build on the third generation and emphasize four aspects: (1) flexible and clean bike stations; (2) bicycle redistribution innovations; (3) the integration of smartcard with other transportation modes; and (4) technological advances, such as GPS tracking, electric bikes, etc. The 3rd and 4th generations of bike-sharing systems have the ability to trace the trip information of bike users so that public bikes can be prevented from theft and vandalism, and all this information is stored in an operational database (CSD, 2011; TransportCanada, 2009). 
Table 1 Generations of bike-sharing programs.

\begin{tabular}{|c|c|c|}
\hline Category & Component & Characteristics \\
\hline \multirow{4}{*}{$\begin{array}{l}1^{\text {st }} \text { Generation } \\
\text { (white bike } \\
\text { systems) }\end{array}$} & \multirow{4}{*}{ Bicycles } & Distinct bicycles (usually by color) \\
\hline & & $\begin{array}{l}\text { Bicycles located haphazardly } \\
\text { throughout an area }\end{array}$ \\
\hline & & Bicycle unlocked \\
\hline & & No charge for use \\
\hline \multirow{3}{*}{$\begin{array}{l}2^{\text {nd }} \text { Generation } \\
\text { (coin-deposit } \\
\text { systems) }\end{array}$} & \multirow{3}{*}{$\begin{array}{l}\text { Bicycles and } \\
\text { Docking stations }\end{array}$} & $\begin{array}{l}\text { Distinct bicycles (color or special } \\
\text { design) }\end{array}$ \\
\hline & & $\begin{array}{l}\text { Bicycle located at specific docking } \\
\text { stations }\end{array}$ \\
\hline & & Bicycles with locks \\
\hline \multirow{6}{*}{$\begin{array}{l}3^{\text {rd }} \text { Generation } \\
\text { (IT-based } \\
\text { systems) }\end{array}$} & \multirow{6}{*}{$\begin{array}{l}\text { Bicycles, } \\
\text { Docking stations, } \\
\text { Kiosks or user } \\
\text { interface }\end{array}$} & $\begin{array}{l}\text { Distinct bicycles (color, special } \\
\text { design, or advertisements ) }\end{array}$ \\
\hline & & $\begin{array}{l}\text { Bicycle located at specific docking } \\
\text { stations }\end{array}$ \\
\hline & & Bicycles have locks \\
\hline & & $\begin{array}{l}\text { Smart technology is used for } \\
\text { bicycle check-in and check-out } \\
\text { (mobile phone, mag-stripe cards, } \\
\text { or smartcards) }\end{array}$ \\
\hline & & $\begin{array}{l}\text { Theft deterrents (program } \\
\text { specific; members are required to } \\
\text { provide ID, bankcard, or mobile } \\
\text { phone number) }\end{array}$ \\
\hline & & $\begin{array}{l}\text { Programs are paid for as a } \\
\text { membership service }\end{array}$ \\
\hline \multirow{7}{*}{$\begin{array}{l}4^{\text {th }} \text { Generation } \\
\text { (demand- } \\
\text { responsive, } \\
\text { multimodal } \\
\text { systems) }\end{array}$} & \multirow{7}{*}{$\begin{array}{l}\text { Bicycles, } \\
\text { Docking stations, } \\
\text { Kiosks-user } \\
\text { interface, } \\
\text { Bicycle distribution } \\
\text { system }\end{array}$} & Distinct bicycles \\
\hline & & $\begin{array}{l}\text { Programs may include electric } \\
\text { bicycles }\end{array}$ \\
\hline & & $\begin{array}{l}\text { More efficient specific docking } \\
\text { stations (mobile, solar powered, } \\
\text { etc.) }\end{array}$ \\
\hline & & $\begin{array}{l}\text { Improved locking mechanism to } \\
\text { detect bicycle theft }\end{array}$ \\
\hline & & Touch screen kiosks-user interface \\
\hline & & Bicycle redistribution system \\
\hline & & Linked to public transit smartcard \\
\hline
\end{tabular}

Source: DeMaio, 2009; Shaheen et al., 2010.

\subsection{Research gaps}

Bike-sharing systems as a pinnacle of sustainable transport systems provide easy access to healthy modes of transport which lead cities away from traffic congestion and air pollution that people currently face. Moreover, these systems relieve its users with costs for bicycle purchase and maintenance cost as well as storage and parking responsibilities. Furthermore, bike- sharing programs have the potential to increase cycling both as a standalone mode 
and for multimodal trips in combination with other public transport modes, especially for large cities where trip distances may not be bikeable on its own. To create a sustainable future for bike-sharing systems, it is essential to understand in more detail how these systems work in a particular local context and how users behave through mining the information embedded in the operational database.

Although bike-sharing systems have attracted worldwide attention, most scientific literature has either focused on user survey-based studies to understand users' profile, perceptions, and travel behaviour (Fuller et al., 2011; O'Neil and Caulfield, 2012; Shaheen et al., 2012; Shaheen et al., 2011; Tang et al., 2011) or on station-based studies to explore the activity patterns of bike stations (Borgnat et al., 2011; Froehlich et al., 2009; Kaltenbrunner et al., 2010; Lathia et al., 2012; Vogel et al., 2011) or the impact of natural and built environment factors (Buck and Buehler, 2012; Ewing and Cervero, 2010; Maurer, 2012; Rixey, 2013). Some studies proposed methods for locating bike rental stations (dell'Olio et al., 2011; García-Palomares et al., 2012; Lin and Yang, 2011). Yet, despite the interesting insights and important implications of these studies, a few research gaps can be identified:

Firstly, unravel travel demand characteristics and patterns of bikesharing systems. Worldwide the adoption of bike-sharing systems in urban transport is booming. Because of its relative short history of existence details of travel demand characteristics and patterns have not been analysed extensively, in particular when considering other urban transport modes. For urban transport planning purposes it is essential to understand the role that bike-sharing systems can play in a particular local context in coherence with the other urban transport systems. The operational databases of the 4th generation systems contain a wealth of information that has hardly been tapped so far. What information can be extracted from these databases, and what are the limitations?

Secondly, identify how built environment factors affect the actual use of public bikes in a Chinese city. The success of bike-sharing systems depends on how users' demand for public bikes is satisfied (Frade and Ribeiro, 2014), and it highly depends on the locations of bike stations (BachandMarleau et al., 2012; Liu et al., 2012; Vogel et al., 2011). However, irrational locations and imbalanced capacity of bike stations have been reported affecting the actual use of bike-sharing systems (Liu et al., 2012; Vogel et al., 2011). As a consequence it leads to a reduced demand of bike-sharing systems. Many studies have stated that the mode choices are strongly associated with built environment characteristics in neighbourhoods (Cervero et al., 2009; Ewing and Cervero, 2010; Kemperman and Timmermans, 2009; Moudon et al., 2005). Moreover, the impact of urban characteristics in neighbourhoods on mode 
choices is much stronger than the impact of subjective variables (personal perceptions), i.e. personal perceptions (Van Acker et al., 2013). Chinese cities being so difference from cities in the western world in urban characteristics, that causes the necessity to investigate how spatial factors drive BSS demand in the context of a Chinese city, to be able to plan successfully new bike-sharing systems or stations in those cities.

Thirdly, analyse travel demand dynamics of bike-sharing systems. Although bike-sharing systems have been running for years in many cities, previous studies neither considered the dynamics of bike-sharing systems over the years nor studied the impact of system expansion on its performance. However, systems and their usage might not be invariable, and may change over the years. Changes over time do not only occur in demand (e.g. for bikes, stations), but possibly also in its users. Examining changes in usage of a system over time enables the identification of factors that influence the system's performance, and can serve as input for improving the locationallocation and planning of new stations.

Finally, mine travel behaviour of bike-sharing users based on the operational database. Most previous studies investigated travel behaviour of bike-sharing users by interviews and a questionnaire survey on a small sample of bike-sharing users. However, very little research employed the information embedded in the operational database to uncover the travel behaviour of users, i.e. when, where, and how many people used the system. Using the operational databases enables researchers and planners to get a complete picture of the use of the system and to explore travel behaviour in a continuous, large scale, and non-invasive way (Beecham and Wood, 2014). Apart from the information of each trip, data can also be used to analyse trip chains and transition activities between consecutive trips.

\subsection{Research objectives and questions}

This research aims to explore methods and approaches to extract information on the actual use of a bike-sharing system and the performance of the system by employing datasets selected from the operational database from the bikesharing system in the city of Zhongshan, China. The empirical research has been focused on the following sub-objectives and questions:

\subsubsection{Objective 1}

To examine the overall characteristics of bike-sharing usage in terms of system efficiency, trip characteristics, and station activity patterns.

\section{Questions:}

- What metrics can be used for evaluating the system efficiency? 
- How bike-sharing trips distribute in terms of O-D patterns and trip duration?

- What are the hourly pickup and return activities at stations?

\subsubsection{Objective 2}

To examine the impact of built environment factors on the usage of public bikes at stations.

\section{Questions}

- How to define and select built environment factors?

- What is the statistical relation between built environment and public bike use in the city of Zhongshan?

\subsubsection{Objective 3}

To examine the dynamics of the bike-sharing system based on the expansion of the system.

\section{Questions}

- How do users and their demands change across the urban area over time?

- How does the expansion of the system affect its use?

\subsubsection{Objective 4}

To explore the travel behaviour of bike-sharing users based on the trip data.

\section{Questions}

- What aspects of bike-sharing trip data can be used for mining the travel behaviour of bike-sharing users?

- What are the spatial and temporal patterns of bike-sharing use?

\subsection{Study area}

In this research the city of Zhongshan, China has been selected as case study to develop methods and approaches to extract information from the operational database to gain a better understanding of the actual use of the bike-sharing system, in terms of overall characteristics, influential factors, dynamic changes, and travel behaviour. A detailed introduction of the city Zhongshan and its transport conditions is presented in following chapters.

There are two main reasons for choosing this study area. First, the largest amount of bike-sharing programs is running in China but only a few researches can be found in scientific literature. Even up to 2016, bike-sharing studies in Asia are rare (Fishman, 2016). The majority of studies dealt with American and European systems, and its findings cannot be transferred to China because of differences in cycling demand and urban features. In comparison with 
American and European cities, Asian cities exhibit quite different urban features, such as mixed land use patterns, and homogeneous compact neighbourhoods, etc. By analysing and visualizing the use of bike-sharing systems in a different urban context, researchers, planners and policy makers can extend and improve their knowledge about such systems. This knowledge is useful for the improvement and expansion of existing systems, as well as the adoption of new systems. Current guidelines on designing bike-sharing systems are the same worldwide, and do not reflect local conditions. Secondly, data stored in operational databases are often confidential, since operations are run by private companies. This study has been privileged by the support of and the provision of temporal selections of the database by the Transport Department of the Urban Planning and Design Institute of Zhongshan. Without this cooperation this study would not have been possible. The database consists of the following items for each trip made: user ID, bike ID, pickup and return station, and start and end time of the trip. For privacy reasons the user ID has been coded.

\subsection{Thesis outline}

This thesis is built around a collection of four papers that have been published or submitted to peer-reviewed scientific international journals. Each of these publications is mentioned on the first page of the corresponding chapter. To maintain a consistent style throughout the thesis, the abbreviations, names, and referencing styles have been standardized, and may slightly differ from those in the originally published papers.

Chapter 2 investigates the overall characteristics of bike-sharing use in the case study area, in terms of system efficiency, characteristics of individual trips, and the spatial and temporal activity patterns of bike use at the level of bike stations. This analysis is based on 5-month trip data of the system from February to June 2014. (Objective 1)

Chapter 3 explores how built environment factors affect the use of public bikes at station level. The chapter employs a spatial multiple linear regression model to examine and elaborate the statistical relations between built environment variables and the actual use of public bikes at stations. Trip demand, and the demand to supply ratio, both at bike station level, are the two dependent variables that have been estimated. Again the same dataset has been used, the 5-month trip data from February to June 2014. (Objective 2)

Chapter 4 explores the dynamics of the bike-sharing systems both from a temporal and spatial perspective. The chapter employs spatial and statistical analyses to examine how users and system usage change over the years, and how the expansion of the system affects the usage of the system. This analysis was conducted using trip data from March 2012, March 2013, and March 2014. 
The number of stations in the system has gradually been expanded between March 2012 and March 2013, and again between March 2013 and March 2014. (Objective 3)

Chapter 5 explores travel behaviour of the bike-sharing users. The chapter examines the spatial patterns of bike-sharing trips, trip chains, and transition activities. The analysis incorporates information of land use types around bike stations, time information of pickup and return actions, and transition activities in between bike trips. Again it is based on the 5-month trip data from February to June 2014. (Objective 4)

Chapter 6 summarizes and synthesizes the main findings of the previous chapters and reflects on its implications. Finally, recommendations for future work are presented. 


\section{Chapter 2}

\section{The characteristics of bike-sharing usage ${ }^{1}$}

\footnotetext{
1 This chapter is based on Zhang, Y., Thomas, T., Brussel, M.J.G. and van Maarseveen, M.F.A.M. (2017) The characteristics of bike-sharing usage : case study in Zhongshan, China : open access. In: The international journal of transport development and integration, 1(2017)2, p. 245-255.
} 


\begin{abstract}
Public bike systems have grown in popularity and are expanding rapidly across cities worldwide. Such systems provide access to pick-up and drop-off public bikes at numerous bike stations for free or for an affordable fee, and aim at increasing bike use and extending the accessibility of traditional public transport systems. A variety of studies have examined the characteristics of bike-sharing systems, mostly in American and European cities and with a focus on user demographics. The objective of this study is to investigate the general characteristics of system usage, in terms of system efficiency, trip characteristics, and bike activity patterns, for Zhongshan's public bike system during a 5-month period. The findings show that the system is not very efficient based on usage metrics which are low compared to successful systems in other countries. Demand is relatively high in city centre zones due to the high population and activity density. However, there is no clear direction of inbound or outbound trips in rush hours. This may be attributed to mixed land use patterns throughout the city, but also to the fact that most trips are local trips over short distance. This could indicate that public bike trips are mainly substitute for walking trips rather than for car or PT trips. On the outskirts, demand and system efficiency are low, indicating that location-allocation of stations needs adjustment. In the conclusions, we discuss how these findings can be used for improving the system.
\end{abstract}

Keywords: Bike-sharing, System efficiency, trip characteristics, bike activity patterns. 


\subsection{Introduction}

Emerging as an innovative and green mode of public transport, public bike systems provide public access to rent and return bikes at numerous bike stations across an urban area. Such systems not only act as a unimodal system, but also contribute to bridging the gap in urban transport systems to solve the "last mile" problem (Liu et al., 2012). Growing concerns about fast motorization and urban pollution, have led to increasing interests in bikesharing programs across the world, from Europe to America, Asia, and Australia (Shaheen et al., 2010). As a result of technological advances and innovations, modern bike-sharing programs enable users to monitor the number of available bikes and parking slots via real-time online maps or mobile apps, and generate station-based or trip-level usage data (O'Brien et al., 2014). Currently, approximately 600 bike-sharing systems are in operation, and many systems are planned for the near future (ITDP-China, 2015).

Some early studies have conducted surveys to analyse the operation and usage of bike-sharing systems. In cities with successful bike-sharing systems these studies reported reduced car use and an increase in cycling (Shaheen et al., 2010). Some surveys revealed that car owners and Individuals, who like bikesharing programs, tended to use public bikes more often (Bachand-Marleau et al., 2012; Shaheen et al., 2011). The proximity of home locations to bike stations and the ownership of bike-sharing membership have a positive effect on shared bicycle use (Shaheen et al., 2011). The shared bicycles have acted as a competitor to bus transit, walking, autos and taxis (Parkes et al., 2013).

The availability of operational usage data, e.g. station-based data or trip-level data, enabled new research possibilities in recent years. Few studies employed data mining techniques to explore the temporal patterns of bike use, based on station data. Froehlich et al. (2009) clustered stations of Barcelona's public bike system based on bicycle activity, and Kaltenbrunner et al. (2010) extended the former analysis by predicting bicycle activity at Barcelona's stations over the hours of the day. Vogel et al. (2011) explored activity patterns at stations of Vienna's system. Borgnat et al. (2011) examined temporal patterns of bike use on system scale in Lyon, and predicted levels of bike use on a daily and hourly basis. They found that weekday and weekend usage peaks are quite different and that at station level differences in peak usage might be associated with the kind of activities in the direct neighbourhood.

Some studies looked at specific features of a bike-sharing system in a city. By analysing 11.6 million bike-sharing trips, Jensen et al. (2010) found that public bikes compete with the car in terms of speed in downtown Lyon. Based on station data, Jäppinen et al. (2013) indicated that integration of public bikes 
with traditional public transportation can promote sustainable daily mobility in Helsinki. Zhao et al. (2015) highlighted that on weekday, women tend to make multiple-circle trips and spend more time in cycling than men based on trip data from Nangjing, China. Several studies explored London's public bike system. These studies found that two strikes of the London subway increased the number and duration of public bike trips (Fuller et al., 2012), and concluded that easier access to the system can promote weekday commuting and weekend use (Lathia et al., 2012).

Recently, some studies analysed a larger set of bike-sharing systems. O'Brien et al. (2014) proposed several metrics to measure system characteristics and gave a classification of 38 public bike systems worldwide, based on system metrics - such as system size, daily usage, bike occupancy of each station, spatial compactness of bike stations, and average number of available bikes over the time of day. They demonstrated that Asian systems have lower compactness than European/Middle Eastern systems, and that Chinese systems group together based on system metrics. Zhao et al. (2014) analysed the effects of urban population, government expenditure, system size, and operation policy on daily bike use by comparing 69 Chinese bike-sharing systems. They recommended a bike-member ratio of less than 0.2 and the adoption of personal credit and universal cards to access to systems. An ITDP report by Gauthier et al. (2013) developed a guideline for bike-sharing planning based on experiences of best used and most efficient systems worldwide. They discussed and proposed a series of factors related to system performance: for example, the system is considered to be efficient if on average each bike is used four to eight times daily, and the ratio of parking docks to bike is 2-2.5.

However, few scientific studies have been reported that analyse the factual use of a bike-sharing system employing the information embedded in the operational database. This information might be beneficial to further improvement and expansion of existing systems as well as the adoption of new systems.

In this study, we focus on system efficiency, trip characteristics, and bike activity patterns. To analyse the spatial dimension we introduced six zones demarcated by concentric circles based on the distance to the city centre. The study was conducted for the bike-sharing system in Zhongshan, China, using trip data during the 5 month period from February 2014 to June 2014.

The paper is organized as follows. Section 2 briefly describes the study context, the bike-sharing system in Zhongshang, and the available database. Section 3 contains the main results of the analysis with respect to system efficiency, trip 
characteristics and bike activity patterns at station level. Finally, section 4 presents the main conclusions of the study.

\subsection{Research context}

\subsubsection{Study context}

In China, bicycle evolution has gone through four phases from initial entry and slow growth (1900s-1978), to rapid growth (1978-1995), bicycle use decrease (1995-2002), and policy diversification (2002-present) (Zhang et al., 2014). This transition is associated with rapid motorization and a gradually deteriorating cycling environment, as a result of economic growth, fast urbanization, and a negative attitude of governmental transport policies towards cycling (Zhang et al., 2014). To alleviate serious traffic congestion and air pollution, China's planners and decision-makers have gradually shifted their focus from offering additional road space for motorized vehicles to examining the need for sustainable transportation strategies (Zhao et al., 2014). Two innovative bicycle forms emerged: electric bikes and public bike systems (Zhang et al., 2014). Along with adopting and advocating the policy on "Transit Priority" that emphasizes the development and improvement of public transport systems, public bike programs have been set up that expanded rapidly although the history of such systems is still short in China. At present, more than 160 public bike systems are running in China, and more and more systems will be in operation in the near future.

\subsubsection{Study area and data preparation}

Zhongshan city is a prefecture-level city that is located in the Guangdong province of China, directly opposite to Hong Kong. The city government administers 6 districts covering the urban area, and 18 towns (Figure 3(A)). Zhongshan's bike-sharing system was launched in 2011 and is a 24/7 selfservice system. After registration, users can pick up and return public bikes at any station during the day, using a smart membership card. For each trip, the first hour is free, and any extra hours are charged at an incremental price (1 CNY per hour), which is much cheaper than a trip by local public bus ( 2 CNY per trip). The system gradually expanded from the central area to the distant industrial district, and further expansion over the city is ongoing.

Trip data from February 2014 to June 2014 were provided by the Transport Department of the Urban Planning and Design Institute of Zhongshan. In June 2014, according to the trip database, 296 bike stations equipped with 7855 parking docks were distributed over the urban area, implying an average station size of 26.5 docks. As shown in Figure 3(B), 224 of these stations were built before 2014, mainly located in the "major urban area", and in 201472 bike stations were built predominantly located in "Torch Hi-tech Industrial 
Development district". The average distance between neighbouring stations is $377 \mathrm{~m}$.

In the trip database each trip record includes user ID, bike ID, pickup and return station, and start and end time of the trip. During the 5 month period the original trip database contained 1937265 records (i.e. trips). In the data cleaning process, $6 \%$ of the records had to be excluded for trips that lasted less than 1 minute mostly starting and ending at the same station. As a result, 1820954 valid records were obtained.

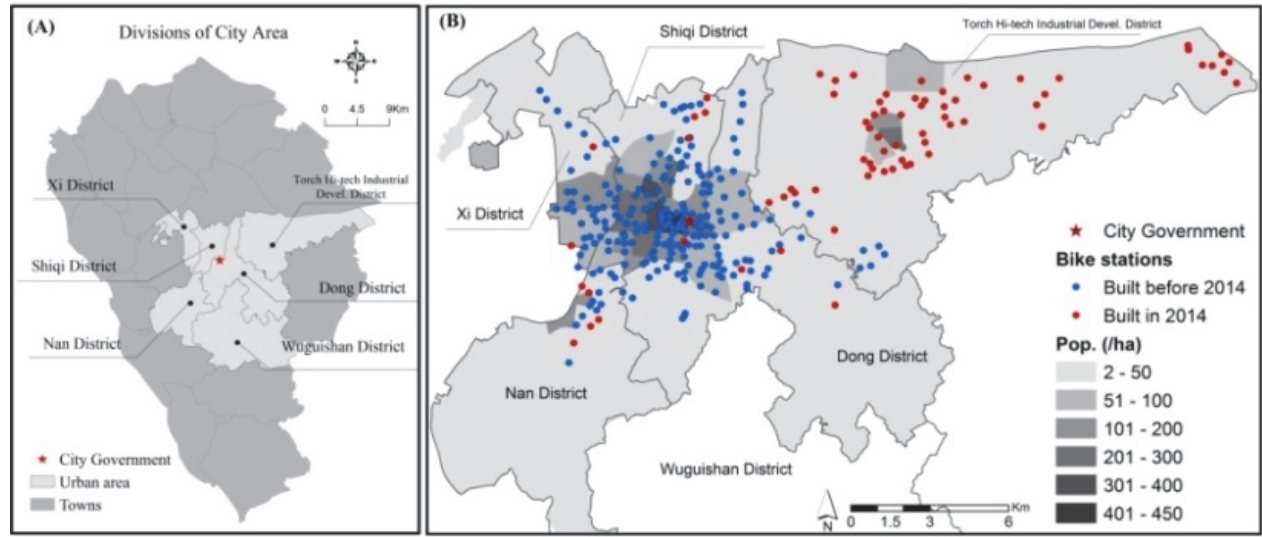

(A)Map of city area; and (B) Spatial distribution of bike stations and population density in the urban area.

Figure 3 City Background.

\subsubsection{Characteristics in bicycle use: the user perspective}

Employing the (anonymous) "User-ID", users have been classified into five groups - User1, User2, User3, User4, and User5 - based on the number of months within the observation period the person used the system. For example, User 3 represents persons that used the system in 3 out of the 5 months. User 1 and User5 account for $27 \%$ and $23 \%$ of the total user population respectively, which is larger than the other user groups. The majority of the User1 group only used the system on weekdays, whereas the majority of all other user groups used the system both on weekdays and in the weekend. The User1 group contains many incidental users, who used the system just once. The more months people are using the system correlates with a higher frequency in number of days per week as well as per month.

This finding suggests two types of users: (1) incidental users, e.g. the User1 group; and (2) regular users, e.g. User3, User4, and User5 groups. Users have to register as a member before they can use the system. This registration procedure might reduce the attractiveness of the system for incidental users. In order to encourage more people to participate in such a system, it would be 
worthwhile to investigate the possibilities to simplify the access procedure. Lathia et al. (2012) showed that introduction of an easier access procedure has raised bike use of London's public bike system.

\subsection{Characteristics of system usage}

A quantitative analysis has been conducted to investigate the characteristics of system usage, in terms of system efficiency, trip characteristics, and bike activity patterns. The analysis looked at the system as a whole as well as at parts of the system spatially to uncover the performance of the system across the urban area. For the spatial analysis, the urban area has been divided into six zones using five concentric rings around the city centre as shown in Figure 4. The six zones cover all bike stations and the vast majority of the urban area. The first ring is the central zone with the highest population density, whereas each successive ring has a lower population density. Zone 6 covers the Hi-tech industrial district and contains the stations newly built in 2014 .

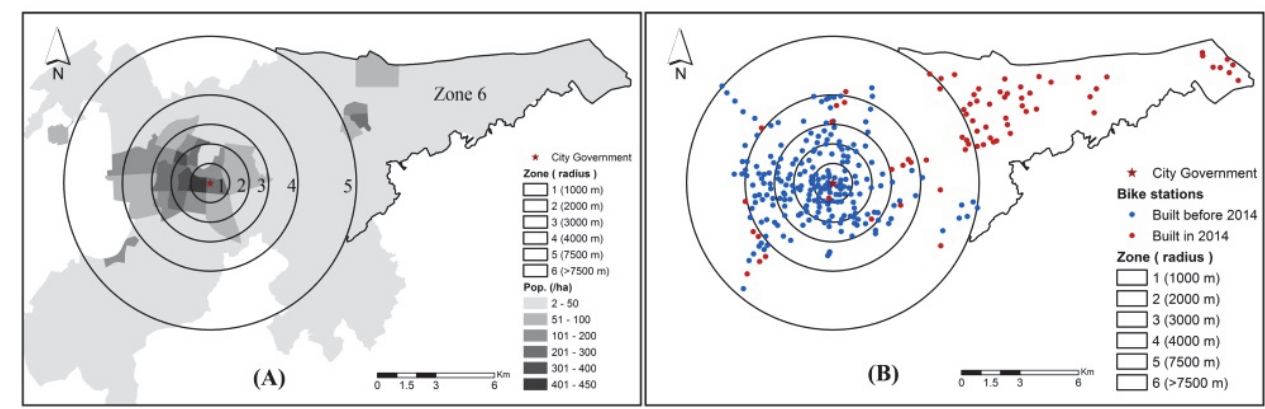

Figure 4 The division of six spatial zones with (A) population density and (B) bike stations in the urban area.

\subsubsection{System efficiency}

Table 2 presents the system metrics at system level as well as for each spatial zone. The total number of bikes is 4072 yielding a slot/bike ratio of 1.9 and on average 5.4 bikes/1000 inhabitants. These values are lower than the ideal values of 2-2.5 and 10-30, respectively, as proposed by Gauthier et al. (2013). It implies that from an efficiency perspective there might be insufficient slots and bikes. Knowing that station density has a positive effect on bike use (Gauthier et al., 2013), the station density of 11 stations $/ \mathrm{km} 2$ in the central zone 1 fits within the bandwidth of $10-16$ stations $/ \mathrm{km} 2$, as suggested by Gauthier et al. (2013). However, all other zones have a substantial lower station density, resulting in a low station density at system level. For simplicity, to get an indication for the number of bikes per 1000 inhabitants for each zone, we assumed a constant slot/bike ratio over the whole area. 
At system level the average daily number of bikes in use is 2543 on weekdays and 2394 on weekends, indicating that only approximately $60 \%$ of all bikes are used every single day. According to the number of daily trips/station in Table 3 , the demand is relatively high in the central zone 1 , and decreases in successive zones. Since the number of stations in the latter zones is higher than in zone 1 , this finding suggests that both bikes and stations are used ineffectively, especially in the zones that is further from the city centre.

Table 2 System metrics.

\begin{tabular}{|l|l|l|l|l|l|l|l|}
\hline & $\begin{array}{l}\text { Whole } \\
\text { System }\end{array}$ & $\begin{array}{l}\text { Zone } \\
1\end{array}$ & $\begin{array}{l}\text { Zone } \\
2\end{array}$ & $\begin{array}{l}\text { Zone } \\
3\end{array}$ & $\begin{array}{l}\text { Zone } \\
4\end{array}$ & $\begin{array}{l}\text { Zone } \\
5\end{array}$ & $\begin{array}{l}\text { Zone } \\
6\end{array}$ \\
\hline Stations & 296 & 33 & 54 & 54 & 63 & 47 & 45 \\
\hline Bikes & 4072 & 424 & 742 & 765 & 870 & 660 & 611 \\
\hline Slots/bike & 1.9 & 1.9 & 1.9 & 1.9 & 1.9 & 1.9 & 1.9 \\
\hline $\begin{array}{l}\text { Station density } \\
\left(/ k^{2}\right)\end{array}$ & 1.24 & 11 & 6 & 3.6 & 1.8 & 0.42 & 0.71 \\
\hline Slots/station & 27 & 25 & 27 & 27 & 27 & 27 & 26 \\
\hline $\begin{array}{l}\text { Bike/1000 } \\
\text { inhabitants }\end{array}$ & 5.4 & 6.3 & 6.7 & 6.4 & 7.8 & 3.5 & 3.8 \\
\hline
\end{tabular}

Table 3 Daily use of the system on weekdays.

\begin{tabular}{|l|l|l|l|l|l|l|l|}
\hline & $\begin{array}{l}\text { Whole } \\
\text { System }\end{array}$ & $\begin{array}{l}\text { Zone } \\
1\end{array}$ & $\begin{array}{l}\text { Zone } \\
2\end{array}$ & $\begin{array}{l}\text { Zone } \\
3\end{array}$ & $\begin{array}{l}\text { Zone } \\
4\end{array}$ & $\begin{array}{l}\text { Zone } \\
5\end{array}$ & $\begin{array}{l}\text { Zone } \\
6\end{array}$ \\
\hline Daily trips & 12871 & 4579 & 6823 & 4734 & 2454 & 902 & 169 \\
\hline $\begin{array}{l}\text { Daily } \\
\text { trips/station }\end{array}$ & 43 & 139 & 126 & 88 & 39 & 19 & 4 \\
\hline Daily trips/slot & 1.64 & 5.60 & 4.77 & 3.21 & 1.46 & 0.71 & 0.14 \\
\hline Daily trips/bike & 3.2 & 10.8 & 9.2 & 6.2 & 2.8 & 1.4 & 0.3 \\
\hline $\begin{array}{l}\text { Daily trips/1000 } \\
\text { inhabitants }\end{array}$ & 17 & 68 & 62 & 40 & 22 & 5 & 1 \\
\hline
\end{tabular}

Gauthier et al. (2013) also proposed values for the performance metrics daily trips per bike (i.e. 4-8) and daily trips per 1000 inhabitants (i.e. 25-50). The former metric indicates efficiency of bike use and the latter market penetration. For zone 1,2 , and 3 the average daily trips/bike is $10.8,9.2$, and 6.2 respectively; and the average daily trips/1000inhabitants is 68,62 , and 40 respectively. Therefore, the three most central zones have both a high efficiency of bike use and a high market penetration comparable to Paris and Barcelona (Gauthier et al., 2013). However, for the peripheral zones 4, 5, and 6 both values are very low, contributing substantially to the low performance values for the overall system, that is, 3.2 daily trips per bike and 17 daily trips per 1000 inhabitants. The low demand in peripheral zones might be attributed to the low station density. It is recommended to investigate whether in these zones an increase in station density potentially combined with a capacity reduction in slots per station might have a positive impact on demand. 


\subsubsection{Trip characteristics}

In terms of trip characteristics, we focused on two aspects: (1) the origindestination pattern of trips, and (2) trip duration. The OD-matrix of public bike trips in Table 4 indicates that there is not a strong orientation of trips towards or from the city centre. The majority of trips are intra-zonal or between adjacent zones. The further away from the city centre, the larger the proportion of intra-zonal trips. In particular in zone 6 , intra-zonal trips account for $86.1 \%$ of the trip generation, posing almost an image of a stand-alone system.

Table 4 O-D matrix of public bike trips (Weekdays).

\begin{tabular}{|l|l|l|l|l|l|l|l|}
\hline O D D & Zone1 & Zone 2 & Zone 3 & Zone 4 & Zone 5 & Zone 6 & Total \\
\hline Zone 1 & $49.89 \%$ & $38.59 \%$ & $9.45 \%$ & $1.76 \%$ & $0.31 \%$ & $0.01 \%$ & $100 \%$ \\
\hline Zone 2 & $25.74 \%$ & $46.19 \%$ & $23.12 \%$ & $4.40 \%$ & $0.53 \%$ & $0.01 \%$ & $100 \%$ \\
\hline Zone 3 & $9.39 \%$ & $32.77 \%$ & $42.04 \%$ & $14.28 \%$ & $1.50 \%$ & $0.03 \%$ & $100 \%$ \\
\hline Zone 5 & $3.01 \%$ & $11.62 \%$ & $26.31 \%$ & $47.87 \%$ & $11.10 \%$ & $0.08 \%$ & $100 \%$ \\
\hline Zone 6 & $1.41 \%$ & $2.87 \%$ & $6.41 \%$ & $30.80 \%$ & $55.60 \%$ & $2.91 \%$ & $100 \%$ \\
\hline
\end{tabular}

Figure 5 shows the distribution of trip durations. Trip duration is defined as the difference between return time and pick-up time of the bike. Distributions are provided for weekdays and weekends (Figure $5(A)$ ), as well as peak and offpeak hours (Figure $5(B)$ ) and average trip times are given for origins and destinations (Figure $5(C)$ ). The distributions are quite similar in all three figures: the average trip duration is less than 16 minutes, most trips last less than 30 minutes and the vast majority of trips finish within one hour (the free of charge period).
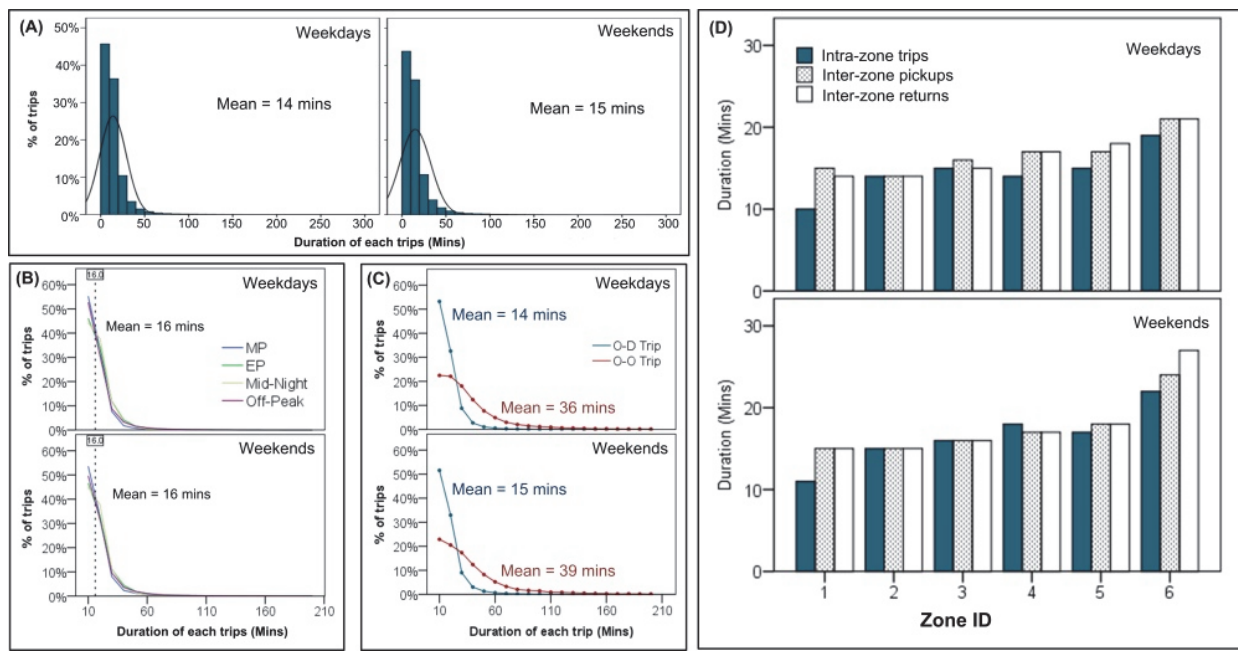

Figure 5 Distribution functions and averages of trip durations. 
According to Figure $5(A)$ and $(B)$, there is no significant difference in trip durations between weekdays and weekends and between peak and off-peak hours. However, the average duration of O-O trips - i.e. trips that start and end at the same station - is 2.5 times longer than the duration of O-D trips (i.e. trips that end at a different station than the pick-up station), as shown in Figure $5(\mathrm{C})$. The presumable explanation is that these trips refer to round trips for which some activity time is included in the trip duration, and the user is not willing or able to dock the bike near the activity location.

Figure 5 (D) shows the average trip duration for intra-zonal trips, inter-zonal pick-ups and inter-zonal returns for each of the zones Except for zone 1, average trip duration for inter-zonal trips is equal to the one for intra-zonal trips, suggesting that origin and destination station for inter-zonal trips are not far from each other. For zone 1 the lower intra-zonal average trip duration is probably due to the high station density in this zone. Figure 5 (D) also shows gradually increasing average trip duration for both intra-zonal and inter-zonal trips the further the distance from the city centre. The most likely explanation is the gradually decreasing station density.

\subsubsection{Bicycle activity patterns at station level}

The analysis on bicycle activity at station level provides insight in both pickups and return activities over the hours of the day. This information could be very valuable in identifying hotspots, for redistribution of public bikes, and planning of the future expansion of the system (Kaltenbrunner et al., 2010). In this study, we performed a hierarchical cluster analysis (Field, 2009) to group the 296 stations based on the temporal patterns of average pickups and returns over the hours of the day. Weekdays have been analysed separately from weekends.

Figure 6 shows the resulting five clusters of bike activity patterns at stations on weekdays. Clearly visible are a morning peak between 7 and 9 AM, an evening peak between 5 and 7 PM, as well as a small lunch peak between 12 AM and 2 PM, when some people go home for lunch and rest, a conventional lifestyle in China in medium and small sized cities.

Cluster 1 , accounting for $63 \%$ of all stations, shows a moderate pattern of morning pickups and evening returns with a higher pickup activity in the morning peak and a higher return activity in the evening peak. The differences in peak activity are in both cases some $5 \%$ of the daily total.

Cluster 2 with $22 \%$ of all stations features an opposite pattern to cluster 1 . These stations show a higher return activity in the morning peak, and the other way around in the evening peak. Again the differences in peak activity are some $5 \%$ of the daily total in both cases. 
Both cluster 3 and cluster 5 show a dominant return activity in the morning peak and a dominant pickup activity in the evening peak. The differences in peak activity are the highest among all clusters and substantial: some $35 \%$ for cluster 3 and $20 \%$ for cluster 5 .

Cluster 4 with the smallest number of stations appears to be a special case. It shows a moderate dominant return activity in the morning peak whereas both pickup and return activities in the evening peak are high. There are two peaks of pickup activity in the evening, and both account for $20 \%$ of daily pickups from the station.
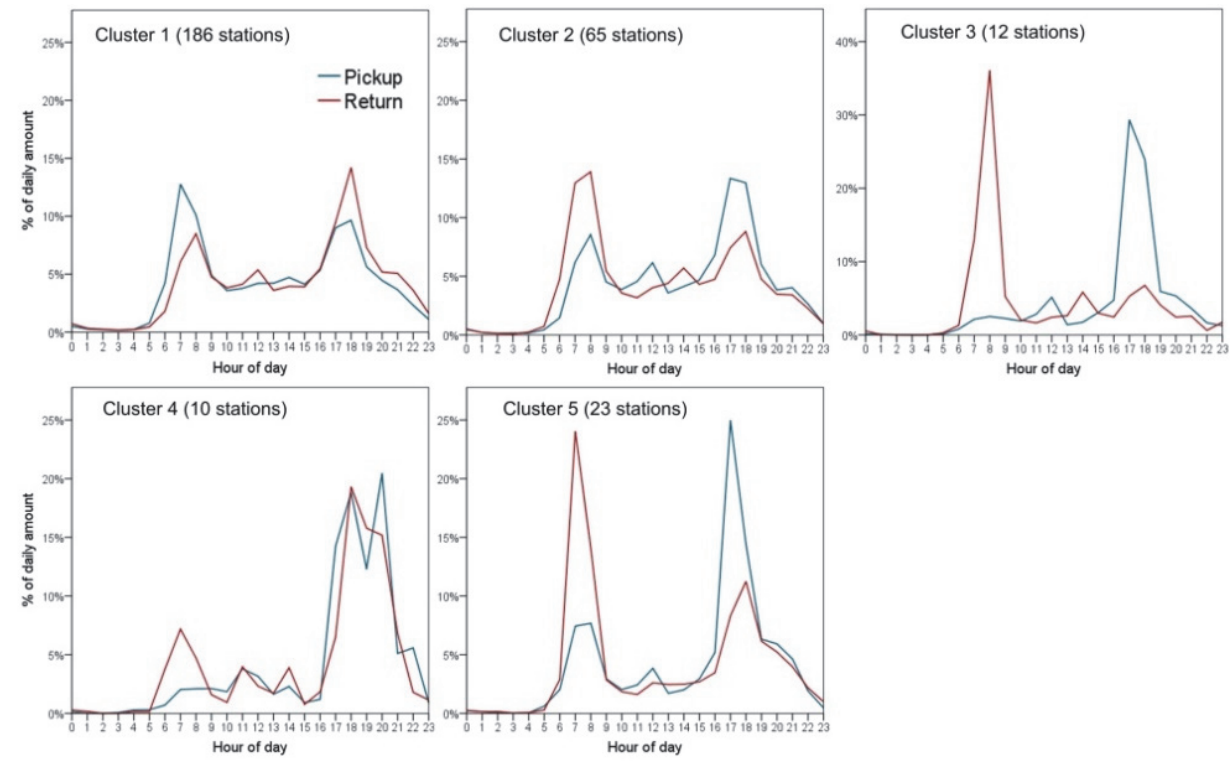

Figure 6 Weekday patterns of bicycle activity at stations.

Figure 7 shows the spatial distribution of these station clusters. The larger clusters 1 and 2 are spread over all zones, except zone 6 . These stations are located in areas with mixed land use patterns and the predominant land use type determines to which of the two activity patterns the station is clustered. The central zones 1 and 2 are primarily occupied with stations of cluster 1 and cluster 2 . Cluster 3 and 5 can be found mainly in the peripheral zones 4,5 and 6. These stations are located in the neighbourhood of shopping malls, offices, and colleges/schools, for which the majority of users arrives for work in the morning peak and leaves for home in the evening peak. The small and peculiar cluster 4 relates to zone 6 , the Hi-tech industrial district, where land use differs substantially from the other areas.

In addition, weekend patterns were classified into 4 clusters, which are comparable with the first four clusters for weekday patterns. Although morning and evening peaks are also visible in weekends, the share of daily trips in peak 
hours in weekends is some $5 \%$ less than on weekdays In contrast to western cities that show commuting on weekdays and leisure trips in weekends, many people in Chinese cities commute both on weekdays and in weekends ( $\mathrm{O}^{\prime}$ Brien et al., 2014; Zhao et al., 2015).

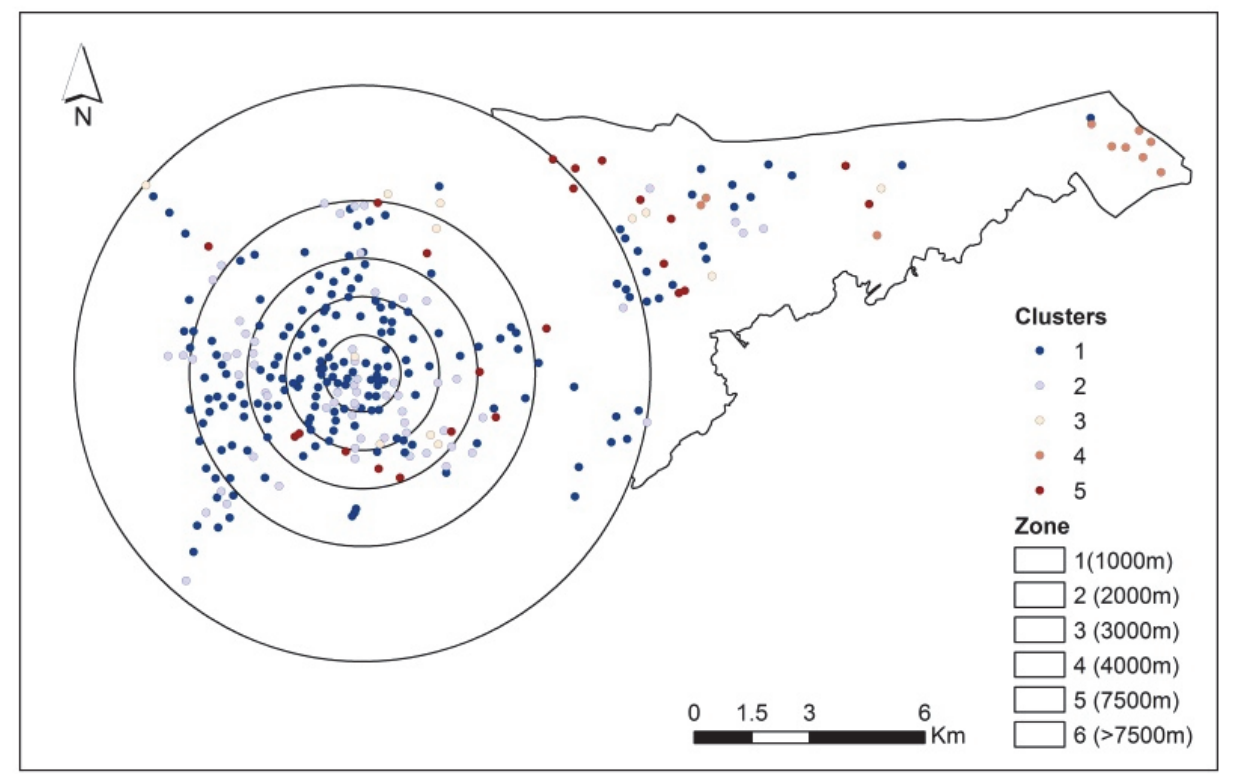

Figure 7 Spatial distribution of station clusters.

\subsection{Conclusions}

This paper investigates characteristics of system usage, in terms of system efficiency, trip characteristics, and bike activity patterns, using operational trip data for a 5-month period of Zhongshan's public bike system. These characteristics were analysed on system level as well as at a disaggregated level of 6 concentric zones.

The overall system is not very efficient in comparison with metrics derived from a global analysis by Gauthier et al. (2013). This finding is mainly attributed to the low bike use and low market penetration in peripheral zones. To improve system efficiency, an increase in station density in these areas, possibly combined with a reduction in station capacity, would be worthwhile to consider.

Public bike demand is relatively high in central zones, where population density is the highest and urban activities are concentrated. However, there is no specific orientation of trips to or from the city centre. The majority of public bike trips are short distance trips within or between adjacent zones. This finding suggests that public bike trips mainly substitute for walking trips rather than for car or public transport trips. Moreover, there is no clear direction of inbound or outbound trips in rush-hour. In contrast to western cities, weekdays 
and weekends show similar patterns for many people in China commute both on weekdays and in weekends (O'Brien et al., 2014; Zhao et al., 2015).

Finally, two types of users need to be distinguished based on the frequency of bike use, that is, "incidental users" and "regular users". From a policy perspective, simplifying the access procedure for "incidental users" might encourage more people to use the system and to improve the turnover of bike use. Lathia et al. (2012) has shown that the introduction of an easier access procedure has raised bike use of London's public bike system. 


\section{Chapter 3}

\section{The impact of built environment factors on the bike use at bike stations ${ }^{2}$}

2 This chapter is based on Zhang, Y., Thomas, T., Brussel, M., van Maarseveen, M. (2017). Exploring the impact of built environment factors on the use of public bikes at bike stations: Case study in Zhongshan, China. Journal of Transport Geography, 58, 5970. 


\begin{abstract}
Many countries have implemented bike-sharing systems to promote sustainable public transportation. Despite the rapid development of such systems, few studies have investigated how built environment factors affect the use of public bikes at station level using trip data, taking account of the spatial correlation between nearby stations. Built environment factors are strongly associated with travel demand and play an important role in the success of bike-sharing systems. Using trip data from Zhongshan's bikesharing system, this paper employed a multiple linear regression model to examine the influence of built environment variables on trip demand as well as on the ratio of demand to supply (D/S) at bike stations. It also considered the spatial correlations of BSS usage between nearby stations, using the spatial weighted matrix. These built environment variables mainly refer to station attributes and accessibility, cycling infrastructure, public transport facilities, and land use characteristics. Generally, we found that both trip demand and the ratio of demand to supply at bike stations were positively influenced by population density, length of bike lanes and branch roads, and diverse landuse types near the station, and were negatively influenced by the distance to city centre and the number of other nearby stations. However, public transport facilities do not show a significant impact on both demand and D/S at stations, which might be attributed to local modal split. We also found that the BSS usage at stations is positively associated with usage at nearby stations. Model results also suggest that adding a new station (with empty capacity) within a $300 \mathrm{~m}$ catchment of a station to share the capacity of the bike station can improve the demand-supply ratio at the station. Referring to both trip demand models and D/S models, regression fits were quite strong with larger R2 for weekdays than for weekends and holidays, and for morning and evening peak hours than for off-peak hours. These quantitative analyses and findings can be beneficial to urban planners and operators to improve the demand and turnover of public bikes at bike stations, and to expand or build bike-sharing systems in the future.
\end{abstract}

Keywords: bike-sharing systems, trip data, trip demand, demand-supply ratio, bike stations, built environment factors 


\subsection{Introduction}

Bike-sharing systems have become popular in many cities, in order to increase cycling, improve the accessibility of traditional public transport, and lessen the impact of motorized transport activities (Kaltenbrunner et al., 2010; Lin and Yang, 2011). Public bike programs provide public access to pick up and return bicycles at numerous fixed bike stations for free or against a small fee across an urban area. With these benefits and the improvement of operation and techniques on tracking public bikes, it has given birth to the rapid expansion of public bike programs throughout Europe, America, and Asia in recent years (Shaheen et al., 2010). Currently, there are more than 600 pubic bike programs across the world, of which around 162 are in China (ITDP-China, 2015). Some studies have shown that cycling has increased in cities after building bike-sharing systems (Pucher et al., 2010).

Convenience and low travel cost have been perceived by users as major perceived benefits of bike-sharing systems(Fishman et al., 2013). Bike-sharing systems can not only offer rapid and flexible mobility for short distance trips, but also serve as a feeder mode for other public transport to improve the accessibility and reduce travel time of other public transport (Jäppinen et al., 2013). A bike-sharing system mainly consists of numerous public bikes, fixed bike stations, and information service terminals. The success of bike-sharing systems depends on how the users' demand for public bikes is satisfied (Frade and Ribeiro, 2014), and this highly depends on the location of bike stations (Bachand-Marleau et al., 2012). However, irrational locations and imbalanced capacity of bike stations have been reported as affecting the actual use of bikesharing systems (Liu et al., 2012; Vogel et al., 2011). This leads to a reduced demand of bike-sharing systems. It is therefore important to know which (spatial) factors drive BSS demand, before planning the allocation of new BSS stations.

Previous studies have shown that the bike-sharing ridership at station-level is associated with the surrounding built environment characteristics (Buck and Buehler, 2012; El-Assi et al., 2015; Ewing and Cervero, 2010; Faghih-Imani and Eluru, 2015, 2016; Faghih-Imani et al., 2014; Gonzalez et al., 2016; Maurer, 2012; Rixey, 2013; Wang et al., 2016), and with the kind of activities that take place in the neighbourhood (Borgnat et al., 2011; Kaltenbrunner et al., 2010; Vogel et al., 2011). According to these studies, surrounding built environment characteristics mainly refer to population and job density, proximity to transit stations (metro and public bus stations) and bike lanes, and point of interests (retail shops, parks, restaurants, etc.) within the service area of each station. Moreover, station size, and number of bike stations within catchment area also have impact on the BSS demand at stations (El-Assi et al., 2015; Faghih-Imani and Eluru, 2015; Faghih-Imani et al., 2014). Most of 
aforementioned studies, except one from Faghih-Imani and Eluru (2016), employed traditional (non-spatial) regression analysis to examine the factors determining BSS demand, but did not consider spatial interaction between nearby stations. This may provide a distorted picture, as nearby stations may not be independent from each other. For example, users might shift from a station to nearby stations when the station is full or empty (Rudloff and Lackner, 2014).

The objective of this study is to understand how built environment factors affect the actual use of BSS. To this end, we examined the statistical relations between built environment variables and the actual use of public bikes at bike stations. Trip demand, i.e. the number of bikes picked up and dropped off at stations, and the demand to supply (supply being the number of parking slots) ratio were computed as two different indicators to represent the usage of public bikes at bike stations and were analysed separately. We used spatial regression analysis to examine how surrounding built environment affects the system usage at stations, including the spatial interaction between nearby stations. The built environment factors - i.e. station attributes and accessibility, cycling infrastructure, public transport facilities, and land use characteristics within the potential service area of each station - are considered as explanatory variables. This quantitative analysis was done for a bike-sharing system in Zhongshan which is a medium sized city in China, using trip database that includes the time and station location of pick-up and return of each trip from February to June 2014.

The rest of this chapter is organized as follows. Section 3.2 gives a brief overview of relevant earlier studies, and in this context explains how this study was conducted. Section 3.3 introduces the study area, and presents the data screening. Section 3.4 describes the definition and generation of dependent and explanatory variables and model development. Section 3.5 presents results and discussion of the statistical models. Finally, section 3.6 summarizes the paper.

\subsection{Literature review}

Many studies have stated that the mode choices are strongly associated with built environment characteristics in neighbourhoods (Cervero et al., 2009; Ewing and Cervero, 2010; Kemperman and Timmermans, 2009; Moudon et al., 2005). Moreover, the impact of urban characteristics in neighbourhood on mode choices is much stronger than the impact of subjective variables (personal perceptions) (Van Acker et al., 2013). A well-known measurement of built environment factors is "D variables". The original three "D variables" coined by Cervero and Kockelman (1997) - are density (population or job density), diversity (land-use mix), and design (street network characteristics), followed later by two additional variables - distance to transit, and destination 
accessibility (Ewing and Cervero, 2010). All these variables should be measured in a given area, i.e. neighbourhood (Ewing and Cervero, 2010). Higher density, more diversity, and better accessible neighbourhood (close to public transport, shops, leisure activities, etc.) (Ewing and Cervero, 2001; Van Acker et al., 2013), the proximity to bicycle-friendly infrastructure (e.g. bike lanes and trails) (Cervero et al., 2009; Heesch et al., 2015; Krizek and Johnson, 2006; Moudon et al., 2005) and transit stations (e.g. metro stations, and bus stops) (Cervero et al., 2009; Heesch et al., 2015) encourage more cycling. Moreover, street density and route connectivity are positively associated with utilitarian cycling (Cervero et al., 2009). It should be noted that the impact of built environment variables on cycling demand depends on various context variables, such as trip purpose (utilitarian, recreational), urban features (e.g. cities with and without uniformly compact neighbourhoods) (Cervero et al., 2009; Heesch et al., 2015; Moudon et al., 2005). As a result, the relation between built environment variables and bicycle use can vary between different cities. For example, close to bicycle infrastructure and urban amenities (e.g. shops, CBD, etc.) there are key attributes influencing utilitarian cycling but not recreational cycling (Heesch et al., 2015). Most of the built environment factors (e.g. density, land-use diversity, accessibility to destination and transit stations) show a significant influence on cycling demand in developed countries but not in Bogota, which is attributed to the different urban features between cities of the developing and the developed world (Cervero et al., 2009).

As for bike-sharing systems, there has been a growing attention towards understanding the effect of surrounding built environment characteristics on the bike-sharing ridership at station-level. The selection of built environment factors varies among different studies and these factors were usually analysed in the catchment (buffer) area around each station. In general, some studies found that the population and job density, and the proximity to bike lanes, transit stations (public bus stops, metro stations) and points of interest (e.g. retail shops, restaurants, etc.), within a given area (service area) of each station are positively associated with ridership at stations (Buck and Buehler, 2012; Daddio, 2012; El-Assi et al., 2015; Faghih-Imani and Eluru, 2015, 2016; Faghih-Imani et al., 2014; Gonzalez et al., 2016; Nair et al., 2013; Wang et al., 2016). However, the effect of the built environment factors is not consistent across different studies. Rixey (2013) indicated that the positive effect of bike lanes only becomes significant when including the days with precipitation in the sample, and suggested that income levels and job types should be included when considering population density and job density. Moreover, Maurer (2012) found that population density and bike lanes have no significant relation with bike use. Additionally, several studies also found that station size (the capacity of station) and number of nearby bike stations have an impact on the ridership at stations, while the effects (positive or negative) 
are different among studies (El-Assi et al., 2015; Faghih-Imani and Eluru, 2015; Faghih-Imani et al., 2014). Furthermore, some studies indicated that severe weather conditions and calendar attributes (weekdays, weekends, and holidays) have influence on the system usage (Corcoran et al., 2014; Gebhart and Noland, 2014).

However, most of the aforementioned studies did not consider spatial correlations between nearby stations, which should actually be considered (Rudloff and Lackner, 2014). Also, most studies have not used distance-decay weighting of demographic or job variables within the service area, which could significantly influence the results (Gutiérrez et al., 2011). And finally, most studies were applied in western cities, whereas many new systems are developed in the developing world. In this study, we addressed some of these shortcomings. We used a spatial multiple linear regression model to examine the impact of built environment variables on trip demand as well as the ratio of demand to supply (D/S) at bike stations in a medium-sized Chinese city. China in particular is interesting, as many of the new BSS systems are launched in China. The selection of our built environment variables is based on the characteristics of bike-sharing systems and the built environment factors (e.g. Density, Diversity, Design, Destination, and Distance) discussed by Ewing and Cervero (2010). In our study, spatial variables were analysed within the catchment area of each bike station, and a distance decay function was used to compute population covered by each bike station.

\subsection{Study area and data}

\subsubsection{Study area}

Zhongshan city is a prefecture-level city located in the Guangdong province of China, and directly opposite Hongkong (Figure $8(\mathrm{~A})$ ). The city government directly administers 6 districts corresponding to the urban area, and 18 towns (Figure 8 (B)). Moreover, 4 districts, the $\mathrm{Xi}$, Shiqi, Dong, and Nan districts, constitute the "centre urban area" (172.7km2), in which the Xi, Shiqi and Dong district are the political and cultural centre of the city and are mainly occupied by the service industry and the Nan district holds the manufacturing and service industries. One of the other two districts, the Torch Hi-tech Industrial Development district $(90 \mathrm{~km} 2)$, is a national-level hi-tech industrial development zone, and the Wuguishan district $(113 \mathrm{~km} 2)$ is mainly for tourism and agriculture. As shown in Figure 8 (C), the largest population is in the core of "centre urban area", and less population in the Hi-tech and Wuguishan district. According to the statistics provided by the local transport planning department (before operating the BSS system), in the "centre urban area", the non-motorized modes account for $46.3 \%$ of total trips, in which the share of bike trips is $18.9 \%$. The share of motorcycle and private car trips is $39.8 \%$ and 
$8.5 \%$ respectively, whereas $4.2 \%$ of trips are made by public bus. Referring to the travel time (distance) in the "centre urban area", the average trip length is $2.7 \mathrm{~km}$, and $94.8 \%$ of total trips are less than $30 \mathrm{mins}$; moreover, the average length of walking trips is $0.8 \mathrm{~km}$, and the average length of cycling and public bus is $2.8 \mathrm{~km}$ and $4.8 \mathrm{~km}$ respectively. The statistics indicate that non-motorized modes (walking and cycling) and motorcycle are the main travel modes, the public bus, which is the only form of public transportation, is not attractive to residents, and the trip length and travel time of residents are quite short in this study area.

Zhongshan's bike-sharing system was launched in 2011 and is a $24 / 7$ selfservice system. Users can pick up and return public bikes at any station in the course of day by use of a smart card after membership registration. For each trip, the first hour is free, and the rest of hours are charged at incremental price (1CNY per hour), which is quite a lot cheaper than a trip by local public bus ( 2 CNY per trip). The data were collected from the Transport Department of the Urban Planning and Design Institute of Zhongshan (China). The provided trip database consists of usage information from February to June 2014 (5 months). Each piece of usage information (i.e. each trip) includes user ID, bike ID, pickup and return stations, and start time and end time of the trip. Using the latter, the duration of each trip is calculated by subtracting the start time from the end time. According to the trip database, until June 2014, 296 bike stations, equipped with 7855 parking slots were distributed over the urban area; 224 of these stations had been built before 2014 and are mainly located in the "centre urban area", and 72 bike stations were built in 2014 and are mainly located in "Torch $\mathrm{Hi}$-tech Industrial Development district", as shown in Figure 8 (C). Additionally, the spatial database of the bike-sharing system and urban attributes, such as population density, land use types, urban road network, and public transportation infrastructure, have also been provided by the local transport department. 


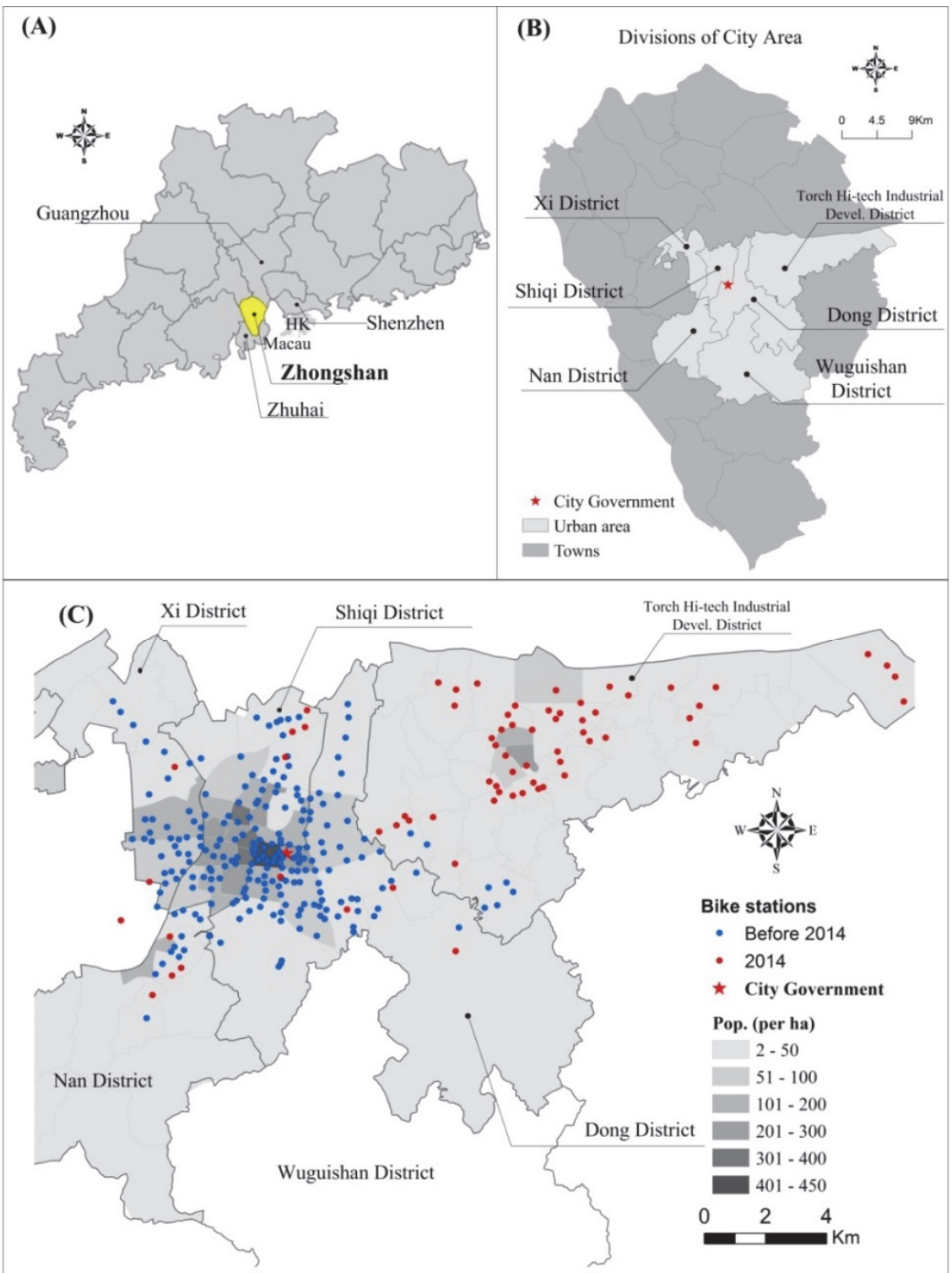

(A) Location of the city in the Guangdong Province; (B) division of the study area; and (C) the spatial distribution of population density and bike stations over the urban area. Figure 8 Background of study area.

\subsubsection{Data preparation}

Referring to the original trip database that records the usage of public bikes from February to June 2014, there are 1,937,265 records (i.e. trips), generated over the urban area in these 5 months. Based on data screening, we excluded $6 \%$ of inaccurate records from the original trip database, which included $5.88 \%$ of trips that had a pickup and return at the same station with a duration of less 
than 1 minute, and $0.12 \%$ of trips that had a duration of less than 1 minute. Moreover, the first two weeks of February 2014 contained Chinese New Year and Lantern Festival. Due to their special character and influence on activity patterns, we decided to exclude these two weeks from the analysis. Furthermore, we excluded newly-built bike stations (built in 2014), because they came on line during the measurement period. Although these newly-built stations were excluded from the regression analysis, they were used to validate the regression model to examine whether model results can also be applied to newly-built stations.

\subsection{Methodology}

\subsubsection{Selection and generation of dependent and explanatory variables}

We employed spatial multiple linear regression analysis to examine the statistical relations between built environment factors and trip demand at bike stations as well as the demand to supply ratio (D/S) at bike stations. The daily demand at a station is given as the average amount of pickups and returns per day (Eq. 3.1). The daily demand to supply ratio (D/S) is equal to this average demand divided by the number of parking slots (supply) of the station (Eq. 3.2). These were used to calculate the daily demand and daily $D / S$ at stations during weekdays, weekends, and holidays. Moreover, we also considered the demand within specific periods of weekdays, i.e. morning-peak (MP, 7:009:00), evening-peak (EP, 17:00-19:00), off-peak (the rest of the hours). The demand is calculated as the average hourly demand at each station (total demand per period divided by the number of hours). The trip demand and D/S were used as dependent variable in different regression models. The descriptive statistics of daily and hourly demand and D/S at stations are shown in Table 5. Note that the number of pickups and returns at each of stations are in general quite comparable as might be expected. 
Table 5 Descriptive statistics of explanatory variables and daily and hourly usage at stations.

\begin{tabular}{|c|c|c|c|}
\hline & Description & Mean & Std. Deviation \\
\hline \multicolumn{4}{|l|}{$\begin{array}{l}\text { Station attributes and } \\
\text { accessibility }\end{array}$} \\
\hline Capacity of the bike station & Number of parking slots of a station & 26.59 & 6.91 \\
\hline $\begin{array}{l}\text { Number of other bike } \\
\text { stations within } 300 \mathrm{~m} \text { buffer }\end{array}$ & $\begin{array}{l}\text { Number of other bike stations within } \\
\text { a } 300 \mathrm{~m} \text { buffer of a station }\end{array}$ & 1.13 & 1.28 \\
\hline $\begin{array}{l}\text { Distance to city } \\
\text { government (m) }\end{array}$ & $\begin{array}{l}\text { The shortest network distance from a } \\
\text { station to city government }\end{array}$ & 3340.02 & 1929.15 \\
\hline $\begin{array}{l}\text { Population within } 300 \mathrm{~m} \\
\text { buffer }\end{array}$ & $\begin{array}{l}\text { The size of population within } 300 \mathrm{~m} \\
\text { buffer of a station based on the } \\
\text { distance decay }\end{array}$ & 16.32 & 15.91 \\
\hline \multicolumn{4}{|l|}{ Cycling infrastructure } \\
\hline $\begin{array}{l}\text { Bike lane within } 1000 m \\
\text { buffer }(m)\end{array}$ & $\begin{array}{l}\text { The length of bike lane within } 1000 \mathrm{~m} \\
\text { buffer of a station }\end{array}$ & 8702.95 & 4483.42 \\
\hline $\begin{array}{l}\text { Main road within } 300 m \\
\text { buffer }(m)\end{array}$ & $\begin{array}{l}\text { The length of main road within } 300 m \\
\text { buffer of a station }\end{array}$ & 563.51 & 362.50 \\
\hline $\begin{array}{l}\text { Secondary road within } \\
300 \mathrm{~m} \text { buffer }(\mathrm{m})\end{array}$ & $\begin{array}{l}\text { The length of secondary road within } \\
300 \mathrm{~m} \text { buffer of a station }\end{array}$ & 550.70 & 396.71 \\
\hline $\begin{array}{l}\text { Branch road within } 300 m \\
\text { buffer }(m)\end{array}$ & $\begin{array}{l}\text { The length of branch road within } \\
300 \mathrm{~m} \text { buffer of a station }\end{array}$ & 1275.41 & 921.49 \\
\hline \multicolumn{4}{|l|}{ Public transport facilities } \\
\hline $\begin{array}{l}\text { Public bus stops within } \\
300 \mathrm{~m} \text { buffer }\end{array}$ & $\begin{array}{l}\text { Number of public bus stop within } \\
300 \mathrm{~m} \text { buffer of a station }\end{array}$ & 1.83 & 1.02 \\
\hline $\begin{array}{l}\text { Distance to the closest } \\
\text { public bus stop }(\mathrm{m})\end{array}$ & $\begin{array}{l}\text { The shortest network distance from a } \\
\text { station to the closest public bus stop }\end{array}$ & 165.47 & 166.01 \\
\hline $\begin{array}{l}\text { Closest stop is a bus } \\
\text { terminal ( } 0 \text { or } 1)\end{array}$ & $\begin{array}{l}\text { The closest public bus stop is a bus } \\
\text { terminal or not }\end{array}$ & 0.058 & 0.234 \\
\hline $\begin{array}{l}\text { Closest stop is a transport } \\
\text { hub ( } 0 \text { or } 1)\end{array}$ & $\begin{array}{l}\text { The closest public bus stop is a } \\
\text { transport hub or not }\end{array}$ & 0.058 & 0.234 \\
\hline \multicolumn{4}{|l|}{ Land use characteristics } \\
\hline $\begin{array}{l}\text { Land use types within } \\
300 \mathrm{~m} \text { buffer }\end{array}$ & $\begin{array}{l}\text { Number of different land use types } \\
\text { within } 300 \text { buffer of a station }\end{array}$ & 3.21 & 1.13 \\
\hline $\begin{array}{l}\text { Near a shopping mall ( } 0 \text { or } \\
1 \text { ) }\end{array}$ & $\begin{array}{l}\text { The station located nearby a } \\
\text { shopping mall or not }\end{array}$ & 0.28 & 0.45 \\
\hline $\begin{array}{l}\text { Near a residential } \\
\text { community }(0 \text { or } 1)\end{array}$ & $\begin{array}{l}\text { The station located nearby a } \\
\text { residential community or not }\end{array}$ & 0.49 & 0.50 \\
\hline $\begin{array}{l}\text { Near a recreational place } \\
(0 \text { or } 1)\end{array}$ & $\begin{array}{l}\text { The station located nearby a } \\
\text { recreational place or not }\end{array}$ & 0.045 & 0.21 \\
\hline Near a park ( 0 or 1$)$ & $\begin{array}{l}\text { The station located nearby a park or } \\
\text { not }\end{array}$ & 0.090 & 0.29 \\
\hline
\end{tabular}

Daily and hourly usage at stations

Min 25 Percentiles50 Percentiles 75 PercentilesMax

\begin{tabular}{lllllll}
\multirow{2}{*}{$\begin{array}{l}\text { Daily demand at } \\
\text { stations }\end{array}$} & Weekdays & 2.67 & 47.89 & 97.27 & 163.33 & 507.05 \\
& Weekends & 2.36 & 41.52 & 78.64 & 136.21 & 475.85 \\
Hourly demand at & Holidays & 2.33 & 38.56 & 69.56 & 118.44 & 430.44 \\
stations & Morning-peak 0.190 & 5.266 & 10.228 & 15.630 & 49.620 \\
& Evening-peak 0.190 & 5.516 & 10.918 & 16.625 & 63.652 \\
Daily D/S at stations & Off-peak & 0.059 & 1.301 & 2.864 & 4.832 & 15.314 \\
& Weekdays & 0.09 & 1.94 & 3.83 & 6.52 & 16.32 \\
Hourly D/S at & Weekends & 0.09 & 1.70 & 3.07 & 5.36 & 13.29 \\
stations & Holidays & 0.11 & 0.38 & 0.99 & 1.57 & 11.24 \\
& Morning-peak 0.00614 & 0.199 & 0.429 & 0.631 & 1.654 \\
& Evening-peak 0.00614 & 0.209 & 0.418 & 0.689 & 2.122 \\
& Off-peak & 0.00226 & 0.054 & 0.109 & 0.196 & 0.480 \\
\hline
\end{tabular}


In Table 5, the explanatory variables considered in this study are shown. These variables were selected based on the characteristics of bike-sharing systems and built environmental factors as discussed by Ewing and Cervero (2010), such as land use diversity, destination accessibility, and distance to transit. Some spatial variables were computed within a buffer area. A buffer of a 300 meter radius around each bike station was considered as an appropriate walking distance, as the distance between two nearby stations is often less than $300 \mathrm{~m}$ and the average trip length is around 10 minutes. We refrained from using the catchment polygon around each bike station generated along the road network, because smaller roads are not complete in our dataset. Based on the complete road network, the calculation of service area using either Euclidean distance or network distance does not seem to decisively affect the results (Gutiérrez et al., 2011). By using a 300 meter buffer around each station, spatial variables were computed at the same resolution for all stations.

The capacity of a station was used to examine the effect of station size (the number of parking slots) on the demand at the station. The number of other bike stations within $300 \mathrm{~m}$ buffer area was calculated to investigate the influence of nearby bike stations. The shortest network distance from a bike station to the location of the city government was calculated to examine the effect of the accessibility to the city centre, as there is not a CBD to represent the main attraction point in centre area. The size of the population within $300 \mathrm{~m}$ buffer area is expected to have a positive impact on the use of public bikes at bike stations, as potentially more people can use the system. The data of population is based on the census data that includes the size of the population in each TAZ (traffic analysis zone). The spatial distribution of population density in each TAZ has been shown in Figure 8 (C). The size of the population within the $300 \mathrm{~m}$ buffer area was computed using a distance decay function (proportional with the inverse of the distance), which means the further away users reside from a bike station the less likely they will use the bike station (Gutiérrez et al., 2011). Each buffer area was divided into six 50m concentric rings. The propensity is constant within each ring and decays outwards, as is shown in Eq. 3.3, where i equals the ID of each bike station, and $\mathrm{j}$ represents each of the six rings. Additionally, it would be ideal to consider both population density and job density, distinguishing between income levels and job types. However, due to data limitation, we were unable to examine the impact of the variables relating to job density, income level, and job types. However, the spatial distribution of population to some extent can be an indication of human activities (e.g. job density) in study area, as jobs and housing are quite mixed in the study area, especially the central urban area with high density of stations.

$$
P D_{i}=\sum_{j=1}\left(P D_{i, j} / d_{j}\right)
$$


Cycling infrastructure variables refer to different levels of roads within the catchment area of each bike station in this study. We mainly considered bike lanes, major roads, secondary roads, and branch roads (the type of urban road is defined by MOHURD (2012)) within the catchment area of bike stations. The length of bike lanes within a 1000-m buffer was computed to identify whether bike lanes have a positive impact on demand at a bike station. We used larger buffer radius here as cycling trips are typically longer $(1300 \mathrm{~m}$ on average for our sample) than walking distances, and are therefore more meaningful than a $300 \mathrm{~m}$ buffer radius when considering the safety in potential cycling areas (El-Assi et al., 2015). The length of major roads, secondary roads, and branch roads within a catchment area were calculated to understand the users' preference for routes (Faghih-Imani et al., 2014). In the urban area, major roads mainly connect each urban district, secondary roads contribute to distributing traffic to local areas, and branch roads mainly connect secondary roads and local communities and serve the traffic in local communities.

Public bikes are expected as a feeder mode for other public transport to improve the service of other public transport. The public bus system is the only traditional public transport in the study area. In order to know how public transport affects the demand and D/S at bike stations, public transport variables considered in this analysis include the number of public stops within the catchment area, the shortest network distance to the nearest public bus stop, and the type of the nearest public bus stop. In the study area, public bus stops are classified in three types: normal stations, terminal stations, and transport hubs. The majority of public bus stops are normal stations. Transport hubs are located in the central area and have a larger potential demand, and terminal stations are mainly located in the remote area and have a lower demand.

Land use characteristics were calculated to capture the impact of land use diversity on the use of public bikes at bike stations. The influence of land use diversity on travel demand has been widely studied in transport studies (Ewing and Cervero, 2010). The number of different land use types within the $300 \mathrm{~m}$ buffer area was calculated to examine how the number of different land use types affects the demand at stations, although the study area has mixed land use patterns. Based on our data (spatial distribution of land use types over urban areas), i.e. the definition of land use types in study area, the land use types contain residential, commercial, educational, recreational, office, and industrial areas (which is mainly distributed on the periphery area and was excluded from calculation). We hypothesized that a higher number will lead to a higher demand, as different land use types might attract larger number of users with different travel purposes than a single land use type. For example, a station that covers both a shopping mall and a residential community can attract users that go for shopping, work in the shopping mall, or live there, but 
a station that covers a residential community can only attract users that live there or visit someone there. In the study area, different land use types are mixed in a street block and are divided into plots, the number of plots (with different land use types) within the $300 \mathrm{~m}$ buffer area is the number of land use types covered by a bike station. Moreover, we also considered the impact of dominant land use types - four attractions related to the land use type that is nearby each bike station: residential communities, shopping malls, recreational places, or parks. This was defined based on the principle of siteselection of bike stations in the study area: If a bike station is near (in front of the entrance of) a residential community, a shopping mall, a recreation place, or a park, then the bike station is named after the nearby residential community, shopping mall, recreational place, or park.

Finally, topography and weather are not considered in this study. Topography is not really an issue as there are no real height differences or other natural barriers that may discourage cycling. Weather conditions were considered as one of the potential factors that could have affected the use of public bikes, but only extreme weather conditions (pouring rain or blistering heat) really discourage cycling (Frade and Ribeiro, 2014). Zhongshan has a subtropical climate with an average temperature of $22{ }^{\circ} \mathrm{C}$ and no extreme temperatures between February and June. Rainfall was not extreme either and did not appear to have a significant influence on bicycle demand. According to the statistical correlation between daily rainfall and daily trips, the number of daily trips was not significantly $(p<0.05)$ influenced by daily rainfall. We therefore did not consider weather conditions in the multiple linear regression analysis.

\subsubsection{Multiple linear regression models}

This section provides a brief description of the multiple linear regression model employed in this study. We firstly employed curve estimation analysis to examine whether the statistical relationship between dependent variable and each explanatory variable is linear or non-linear (e.g. logarithmic, power, exponential, etc.). The result of this analysis indicated that the data of the dependent variable and most of the explanatory variables should be transformed using the natural logarithm model (corresponding with a direct demand model), except for the data of distance to the city government and the categorical variables (see Table 5). This implies that the use of public bikes at a bike station goes down exponentially with the distance to the city government.

To explore the relationship between built environment variables and the BSS usage at stations, we used a spatial regression model with two spatially lagged variables, i.e. the spatially lagged dependent variable and the spatially lagged parking slots. Spatial correlation implies that the demand of one station is correlated with the demand of nearby stations, simply because they are in 
close proximity. Moreover, the demand of one station may also be associated the number of parking slots at neighbouring stations. We therefore also included the spatially lagged parking slots as an extra spatial variable. By using these spatial variables, we are able to examine the spatial correlation between nearby stations and the spatial spillover effect. The spatial regression model is shown in Eq. 3.4.

$$
\ln Y_{s}=\beta_{0}+\beta^{\prime} X_{s[\text { Dis_Govt }]}+\sum \beta_{i} \ln X_{s i}+\sum \beta_{j} X_{s j}+\rho W \ln Y+\rho^{\prime} W \ln X_{\text {slot }}
$$

Where $s(=1,2,3, \ldots)$ is an index to represent each bike station, $\beta \_0$ is a constant, $X_{\text {[Dis_Govt] }}$ is the shortest network distance from station $s$ to the city government and $\beta^{\prime}$ is the corresponding model coefficient. $X_{s j}$ and $\beta_{j}$ are the categorical variables ( $j$ ) and their corresponding coefficients, and $X_{s i}$ and $\beta_{i}$ are the remaining independent variables (i) and corresponding coefficients. The spatial weight matrix $\mathrm{W}$ has elements $\mathrm{W}_{\text {ss' }}$ expressing the potential spatial interaction between a station $s$ and the neighbouring station $s^{\prime}$ in our study. WInY as the spatial lag dependent variable with spatial autoregressive parameter $\rho$, and $W \ln X_{\text {slot }}$ as spatially lagged parking slots with spatial autoregressive parameter $\rho^{\prime}$. W can be computed using contiguity rook and queen, inverse distance, k-nearest neighbours, and so forth (Anselin and Rey, 2014). In our study, the observations are discrete points, which suits the inverse distance (with power 1 ) calculation within a threshold distance. We used $300 \mathrm{~m}$ as threshold distance to calculate the spatial weighted matrix $W$. Moreover, a row-standardization transformation of $W$ is adopted to make the estimation stable. All the spatial models were estimated by using maximum likelihood method in GeoDaSpace software (see Anselin and Rey (2014) for the explanation on software and approaches in detail).

We carried out the standard (non-spatial) multiple linear regression analysis using SPSS, and the spatial multiple regression analysis using GeoDaSpace. In order to have a good quality of output models, as suggested by Field (2009), we firstly ran the regression analysis in which all predictors were entered into the model, and examined the output to know which independent variables contribute significantly $(p<0.05)$ to the model's ability to predict the outcome. Based on these important independent variables, we reran the multiple linear regression analysis using a Forward (stepwise) method that adds each significant $(p<0.05)$ variable step by step, which was done by SPSS automatically and stopped when all the significant variables were included in the model. The analysis output displayed all the steps and the model generated in each step. Although the model generated in the final step has the largest R2 and contains all the significant variables, we still compared each model's result, including several statistics tables (e.g. Model summary, ANOVA, Coefficients, etc.) and standardized residual plots, to make sure that the final model had the best performance and had a significant fit to the overall data $(p<0.05)$. 
The model estimates for weekdays, weekends, and holidays, as well as morning-peak, evening-peak, and off-peak were analysed separately.

\subsection{Analysis and results}

\subsubsection{Model results}

In this section, we present and discuss the results of the multiple linear regression models. Note that we only show results that are statistically significant, i.e. those variables that contribute significantly to the dependent variable. The results are presented for demand in Table 6 (different days) and Table 8 (periods of the day), and for $\mathrm{D} / \mathrm{S}$ in Table 7 (different days) and Table 9 (periods of the day). The tables indicate that results are quite similar for demand and $D / S$, for different days, and for different periods of the day. The R2 value, and the magnitude (significance) and direction (positive or negative) of the coefficients are quite comparable, although regression fits appear to be somewhat better for the weekdays than for weekends and holidays, and also better for morning and evening peaks than for off-peaks. In the paragraphs, we elaborate on the influence of independent variables on demand and $D / S$.

As expected, bike stations with a higher demand and D/S are located in the centre urban area with the highest population density (similar to findings of Daddio (2012)). The capacity of a bike station has a positive impact on the demand at the station on weekends and holidays (as well as the morning-peak and evening-peak of weekdays). This implies that the demand at a station is not significantly subject to the size of the station during off-peak hours of weekdays, but users prefer to choose the bike station with a larger capacity on weekends and holidays and during the morning-peak and evening-peak of weekdays (similar to the findings of El-Assi et al. (2015)), which might be attributed to the fact that chooseing stations with larger capacity can increase the chance of finding an available slot or bike. Moreover, station capacity shows a negative impact on daily and hourly $D / S$ at stations on weekdays (no influence on weekends and holiday, which is attributed to the $D / S$ calculation (Eq. 3.2) - the magnitude of $D / S$ (demand to supply ratio) varies inversely with station capacity.

The number of other bike stations within $300 \mathrm{~m}$ catchment area has a negative impact on both demand and D/S at the bike station, i.e. the more other bike stations exist within $300 \mathrm{~m}$ catchment of a bike station, the lower the demand and $\mathrm{D} / \mathrm{S}$ generated at this station (same to the findings of daily customer model of Faghih-Imani and Eluru (2015)). This implies that potential competition exists between nearby bike stations. According to the D/S model of weekdays, both station capacity and the number of other stations within $300 \mathrm{~m}$ catchment have negative impact, but the negative impact of station capacity is 1.7 times larger than the negative impact of number of other stations. When keeping 
other variables constant, we find that adding a new station (with empty capacity) within a $300 \mathrm{~m}$ buffer of a station to share the capacity of the bike station can improve the D/S at the station, in other words, relocating the capacity of a station to a new station within $300 \mathrm{~m}$ buffer of the station.

Unsurprisingly, the use of public bikes at bike stations increases when there are more bike lanes built within $1000 \mathrm{~m}$ buffer area of these stations. The length of branch roads nearby a bike station also has a positive impact on the use of public bikes at the station, but the length of bigger roads (main road and secondary road) in the vicinity of a bike station has no statistically significant influence on the use of public bikes at the station (similar to the impact of minor roads indicated by Faghih-Imani et al. (2014)). Apparently, for these types of roads, the positive effect of accessibility is offset by the fact that these roads are not attractive for cyclists. In other words, these results imply that users prefer to cycle on bike-friendly roads and (branch) roads that are more accessible to local communities (e.g. residential, commercial, park, etc.) in the study area.

The number of different land use types within the $300 \mathrm{~m}$ buffer of each station is associated with a positive impact on both demand and D/S at bike stations. This implies that a higher demand and D/S generated at stations in the vicinity of more diverse land use patterns. Stations near a residential community show a higher D/S during weekends and during the off-peak hours of weekdays, and stations near a park show a lower demand and D/S during morning-peak and evening-peak hours of weekdays. This indicates that residential stations have a larger turnover on weekends and during off-peak hours of weekdays, and stations near parks have a lower demand and turnover during morning-peak and evening-peak hours of weekdays.

The public transport variables did not have a statistically significant effect on the use of public bikes at bike stations. We have known that non-motorized modes and motorcycle are the main travel modes before operating a bikesharing system, whereas the public bus system is less attractive to residents. Given that the use of a bike-sharing system is free of charge in the first hour, which is much cheaper than a trip by public bus, the majority of users can choose public bikes to complete their entire trips without the necessity for transferring from/to public bus, which is similar to the use of BSS in Zhuzhou city (a medium-sized Chinese city) (Zhang et al., 2015). This implies that the significant role of a bike-sharing system is not an intuitive feeder mode to exiting public transport system in our study, but serves as a single mode for users to complete the entire trips, which might be attributed to the local modal split and the condition of exiting public transport systems. 
The spatial correlations do exist between neighbouring stations, i.e. the demand at one station is positively correlated with the demand at neighbouring stations (similar to the findings of Faghih-Imani and Eluru (2016)). Both daily and hourly demand (and $\mathrm{D} / \mathrm{S}$ ) at a station are positively correlated with the demand (and D/S) from nearby stations. Moreover, the hourly D/S at a station during weekdays is also positively correlated with the number of parking slots from nearby stations. The positive correlation of demand (and D/S) between nearby stations might be attributed to the reason that nearby stations share the same built environment attributes that result in the high (or low) demand at these stations (Cervero et al., 2009). Another reason might be that, due to the spillover effect of demand from nearby stations. For example, a high demand generated at a station, i.e. near its capacity (no bike or parking slot), users have to shift from this station to a nearby station to seek for an available bike (or parking slot) (similar to the finding of Rudloff and Lackner (2014)).

Table 6 Regression coefficients for estimated models -- dependent variable Ln[D] of weekdays, weekends, and holidays.

\begin{tabular}{|c|c|c|c|c|c|c|}
\hline \multirow{2}{*}{$\operatorname{Ln}[\mathrm{D}]$} & \multicolumn{2}{|l|}{ Weekdays $^{1}$} & \multicolumn{2}{|l|}{ Weekends $^{2}$} & \multicolumn{2}{|l|}{ Holidays $^{3}$} \\
\hline & Coefficient & t-stat & Coefficient & t-stat & Coefficient & t-stat \\
\hline (Constant) & 2.621 & 6.525 & 0.441 & 0.701 & 0.068 & 0.106 \\
\hline Capacity of the station & - & - & $0.599 * * *$ & 4.150 & $0.674 * * *$ & 4.583 \\
\hline $\begin{array}{l}\text { Number of other bike } \\
\text { stations within } 300 \mathrm{~m} \\
\text { buffer }\end{array}$ & $-0.582 * * *$ & -4.531 & $-0.459 * * *$ & -3.429 & $-0.490 * * *$ & -3.637 \\
\hline $\begin{array}{l}\text { Number of population } \\
\text { within } 300 \mathrm{~m} \text { buffer }\end{array}$ & $0.226 * * *$ & 5.325 & $0.223 * * *$ & 4.977 & $0.236 * * *$ & 5.175 \\
\hline $\begin{array}{l}\text { Network distance to city } \\
\text { government }\end{array}$ & $-0.000163 * * *$ & -4.834 & $-0.000127 * * *$ & -3.572 & $-0.000114 * *$ & -3.156 \\
\hline $\begin{array}{l}\text { Length of bike lane } \\
\text { within } 1000 \mathrm{~m} \text { buffer }\end{array}$ & $0.0727 * *$ & 3.279 & $0.0720 * *$ & 3.079 & $0.0608 *$ & 2.557 \\
\hline $\begin{array}{l}\text { Length of branch road } \\
\text { within } 300 \mathrm{~m} \text { buffer }\end{array}$ & $0.0826 * * *$ & 3.355 & $0.103 * * *$ & 3.955 & $0.101 * *$ & 3.836 \\
\hline $\begin{array}{l}\text { Number of land use } \\
\text { types within } 300 \mathrm{~m} \\
\text { buffer }\end{array}$ & $0.544 * * *$ & 3.802 & $0.416 * *$ & 2.758 & $0.455^{*}$ & 2.961 \\
\hline $\begin{array}{l}\text { Spatially lagged } \\
\text { dependent variable }\end{array}$ & $0.1006 * * *$ & 3.358 & $0.0755^{*}$ & 2.329 & $0.0856 *$ & 2.564 \\
\hline
\end{tabular}


Table 7 Regression coefficients for estimated models -- dependent variable Ln[D/S] of weekdays, weekends, and holidays.

\begin{tabular}{|c|c|c|c|c|c|c|}
\hline \multirow{2}{*}{$\operatorname{Ln}[\mathrm{D} / \mathrm{S}]$} & \multicolumn{2}{|l|}{ Weekdays $^{4}$} & \multicolumn{2}{|l|}{ Weekends ${ }^{5}$} & \multicolumn{2}{|l|}{ Holidays $^{6}$} \\
\hline & Coefficient & t-stat & Coefficient & t-stat & Coefficient & t-stat \\
\hline (Constant) & 1.307 & 2.378 & -0.967 & -2.286 & -1.064 & -2.451 \\
\hline Capacity of the station & $-0.4023^{* *}$ & -3.011 & - & - & - & - \\
\hline $\begin{array}{l}\text { Number of other bike } \\
\text { stations within } 300 \mathrm{~m} \\
\text { buffer }\end{array}$ & $-0.4016 * * *$ & -4.430 & $-0.302 * *$ & -3.209 & $-0.322 * * *$ & -3.445 \\
\hline $\begin{array}{l}\text { Number of population } \\
\text { within } 300 \text { m buffer }\end{array}$ & $0.213 * * *$ & 4.970 & $0.216 * * *$ & 4.713 & $0.218^{* * *}$ & 4.610 \\
\hline $\begin{array}{l}\text { Network distance to city } \\
\text { government }\end{array}$ & $-0.000185^{* * *}$ & -5.648 & $-0.000123 * * *$ & -3.438 & $-0.000105^{* *}$ & -2.869 \\
\hline $\begin{array}{l}\text { Length of bike lane } \\
\text { within } 1000 \mathrm{~m} \text { buffer }\end{array}$ & $0.0645^{* *}$ & 3.062 & $0.0687 * *$ & 2.911 & $0.0679 * *$ & 2.835 \\
\hline $\begin{array}{l}\text { Length of branch road } \\
\text { within } 300 \mathrm{~m} \text { buffer }\end{array}$ & - & - & $0.093 * * *$ & 3.562 & $0.101^{* * *}$ & 3.795 \\
\hline $\begin{array}{l}\text { Number of land use } \\
\text { types within } 300 \mathrm{~m} \\
\text { buffer }\end{array}$ & $0.641 * * *$ & 4.663 & $0.501 * * *$ & 3.291 & $0.486 * *$ & 3.148 \\
\hline Near a park & $-0.316^{*}$ & -2.574 & - & - & - & - \\
\hline $\begin{array}{l}\text { Near a residential } \\
\text { community }\end{array}$ & - & - & $0.204 * *$ & 2.716 & - & - \\
\hline $\begin{array}{l}\text { Spatially lagged } \\
\text { dependent variable }\end{array}$ & $0.176 * *$ & 3.148 & $0.121 *$ & 1.955 & $0.153 *$ & 2.448 \\
\hline
\end{tabular}

Table 8 Regression coefficients for estimated models -- dependent variable Ln[D] of MP, EP, and Off-peak.

\begin{tabular}{|c|c|c|c|c|c|c|}
\hline \multirow{2}{*}{$\operatorname{Ln}[\mathrm{D}]$} & \multicolumn{2}{|l|}{ Morning-peak ${ }^{7}$} & \multicolumn{2}{|l|}{ Evening-peak ${ }^{8}$} & \multicolumn{2}{|l|}{ Off-peak ${ }^{9}$} \\
\hline & Coefficient & t-stat & Coefficient & t-stat & Coefficient & t-stat \\
\hline (Constant) & -1.509 & -2.526 & -1.375 & -2.480 & -1.121 & -2.566 \\
\hline Capacity of the station & $0.555^{* * *}$ & 4.005 & $0.597 * * *$ & 4.645 & - & - \\
\hline $\begin{array}{l}\text { Number of other bike } \\
\text { stations within } 300 \mathrm{~m} \\
\text { buffer }\end{array}$ & $-0.498 * * *$ & -4.542 & $-0.453 * * *$ & -4.331 & $-0.339 * * *$ & -3.648 \\
\hline $\begin{array}{l}\text { Number of population } \\
\text { within } 300 \mathrm{~m} \text { buffer }\end{array}$ & $0.131 * *$ & 2.974 & $0.184 * * *$ & 4.476 & $0.215^{* * *}$ & 4.492 \\
\hline $\begin{array}{l}\text { Network distance to city } \\
\text { government }\end{array}$ & $-0.000188^{* * *}$ & -5.498 & $-0.000171 * * *$ & -5.397 & $-0.000143 * * *$ & -3.855 \\
\hline $\begin{array}{l}\text { Length of bike lane } \\
\text { within } 1000 \mathrm{~m} \text { buffer }\end{array}$ & $0.106 * * *$ & 4.794 & $0.0833 * *$ & 4.048 & $0.0736 * *$ & 3.051 \\
\hline $\begin{array}{l}\text { Length of branch road } \\
\text { within } 300 \mathrm{~m} \text { buffer }\end{array}$ & $0.0765 * *$ & 3.097 & $0.0750 * *$ & 3.271 & $0.0915^{* * *}$ & 3.419 \\
\hline $\begin{array}{l}\text { Number of land use } \\
\text { types within } 300 \mathrm{~m} \\
\text { buffer }\end{array}$ & $0.635 * * *$ & 4.421 & $0.531 * * *$ & 3.986 & $0.598 * * *$ & 3.852 \\
\hline Near a park & $-0.363 * *$ & -2.868 & $-0.433 * * *$ & -3.681 & - & - \\
\hline $\begin{array}{l}\text { Spatially lagged } \\
\text { dependent variable }\end{array}$ & $0.149 * *$ & 3.170 & $0.120 * *$ & 2.671 & $0.195 * *$ & 3.248 \\
\hline
\end{tabular}

Table 9 Regression coefficients for estimated models -- dependent variable $\operatorname{Ln}[D / S]$ of MP, EP, and Off-peak.

\begin{tabular}{|c|c|c|c|c|c|c|}
\hline \multirow{2}{*}{$\operatorname{Ln}[\mathrm{D} / \mathrm{S}]$} & \multicolumn{2}{|c|}{ Morning-peak ${ }^{10}$} & \multicolumn{2}{|c|}{ Evening-peak ${ }^{11}$} & \multicolumn{2}{|l|}{ Off-peak ${ }^{12}$} \\
\hline & Coefficient & t-stat & Coefficient & t-stat & Coefficient & t-stat \\
\hline (Constant) & -1.527 & -2.529 & -1.435 & -2.567 & -3.070 & -4.867 \\
\hline
\end{tabular}




\begin{tabular}{|c|c|c|c|c|c|c|}
\hline Capacity of the station & $-0.443 * *$ & -3.183 & $-0.395^{* *}$ & -3.068 & $-0.391 * *$ & -2.695 \\
\hline $\begin{array}{l}\text { Number of other bike } \\
\text { stations within } 300 \mathrm{~m} \\
\text { buffer }\end{array}$ & $-0.488 * * *$ & -3.649 & $-0.415 * * *$ & -3.349 & $-0.453 * *$ & -3.276 \\
\hline $\begin{array}{l}\text { Number of population } \\
\text { within } 300 \mathrm{~m} \text { buffer }\end{array}$ & $0.129 * *$ & 2.864 & $0.176 * * *$ & 4.160 & $0.2496 * * *$ & 5.356 \\
\hline $\begin{array}{l}\text { Network distance to city } \\
\text { government }\end{array}$ & $-0.000187^{* * *}$ & -5.363 & $-0.000168 * * *$ & -5.185 & $-0.000154 * * *$ & -4.296 \\
\hline $\begin{array}{l}\text { Length of bike lane } \\
\text { within } 1000 \mathrm{~m} \text { buffer }\end{array}$ & $0.107 * * *$ & 4.729 & $0.0857 * * *$ & 4.087 & $0.0576 *$ & 2.424 \\
\hline $\begin{array}{l}\text { Length of branch road } \\
\text { within } 300 \mathrm{~m} \text { buffer }\end{array}$ & $0.0767 * *$ & 3.101 & $0.0754 * * *$ & 3.296 & $0.082 * *$ & 3.181 \\
\hline $\begin{array}{l}\text { Number of land use } \\
\text { types within } 300 \mathrm{~m} \\
\text { buffer }\end{array}$ & $0.638 * * *$ & 4.423 & $0.543 * * *$ & 4.062 & $0.603 * * *$ & 4.006 \\
\hline $\begin{array}{l}\text { Near a residential } \\
\text { community }\end{array}$ & - & - & - & - & $0.204 * *$ & 2.696 \\
\hline Near a park & $-0.362 * *$ & -2.846 & $-0.425 * * *$ & -3.609 & - & - \\
\hline $\begin{array}{l}\text { Spatially lagged } \\
\text { dependent variable }\end{array}$ & $0.154 *$ & 2.506 & $0.143^{*}$ & 2.364 & $0.157 *$ & 2.562 \\
\hline $\begin{array}{l}\text { Spatially lagged } \\
\text { variable [Slot] }\end{array}$ & $0.147 * *$ & 2.926 & $0.112 *$ & 2.388 & $0.216 * *$ & 3.284 \\
\hline
\end{tabular}

\subsubsection{Model validation}

Figure 9 displays the model results versus the observed values for demand and D/S at bike stations built before 2014 (blue symbol) and stations built in 2014 (red symbol). The latter ones are used to check the validity of the regression model. Figure 9 indicates that the residuals appear to be somewhat larger for the red stations, but this may be attributed to the fact that these stations are located in the Hi-tech industrial district and serve fewer people. As a consequence, random variation due to limited sample sizes is stronger for these stations. There is however no evidence for systematic overestimation or underestimation by the model. In other words, the model also seems to be valid for the new stations (red symbols in Figure 9).

For the blue stations (stations built before 2014) in Figure 9, the regression fit also seems to be appropriate. No systematic effects are detected when the unstandardized residuals are plotted versus the independent variables (Figure 10 as illustration for the weekdays model). Although model values are not exactly the same as observed values, there is no systematic overestimation or underestimation by the model. Residuals larger than 2 times the standard deviation are shown by the red (square) symbols in Figure 10, while those smaller than 2 times the standard deviation are shown by blue (triangle) symbols. These so-called outliers mostly are the same stations in the different models. To understand the nature of these outliers, the spatial distribution of non-outliers and outliers are shown in Figure 11, these outliers are mainly distributed on the outer layer of "centre urban area". In regard to outliers (bike stations), several independent variables, which did not have a significant contribution in the model results, might have a decisive impact on the BSS use 
at these bike stations in reality. This results in the use of public bikes at bike stations being overestimated or underestimated by the models. For example, one station (outlier) was underestimated by the model due to the fact that the station is far from the centre (e.g. fewer bike lanes, etc.), while the station is located nearby a shopping mall, which possibly leads to a higher demand for these stations than the model expected. Generally speaking, although regression models did not perfectly match all the observations, they still indicate the impact of influential factors and their contributions to increasing or decreasing the use of public bikes at bike stations. 

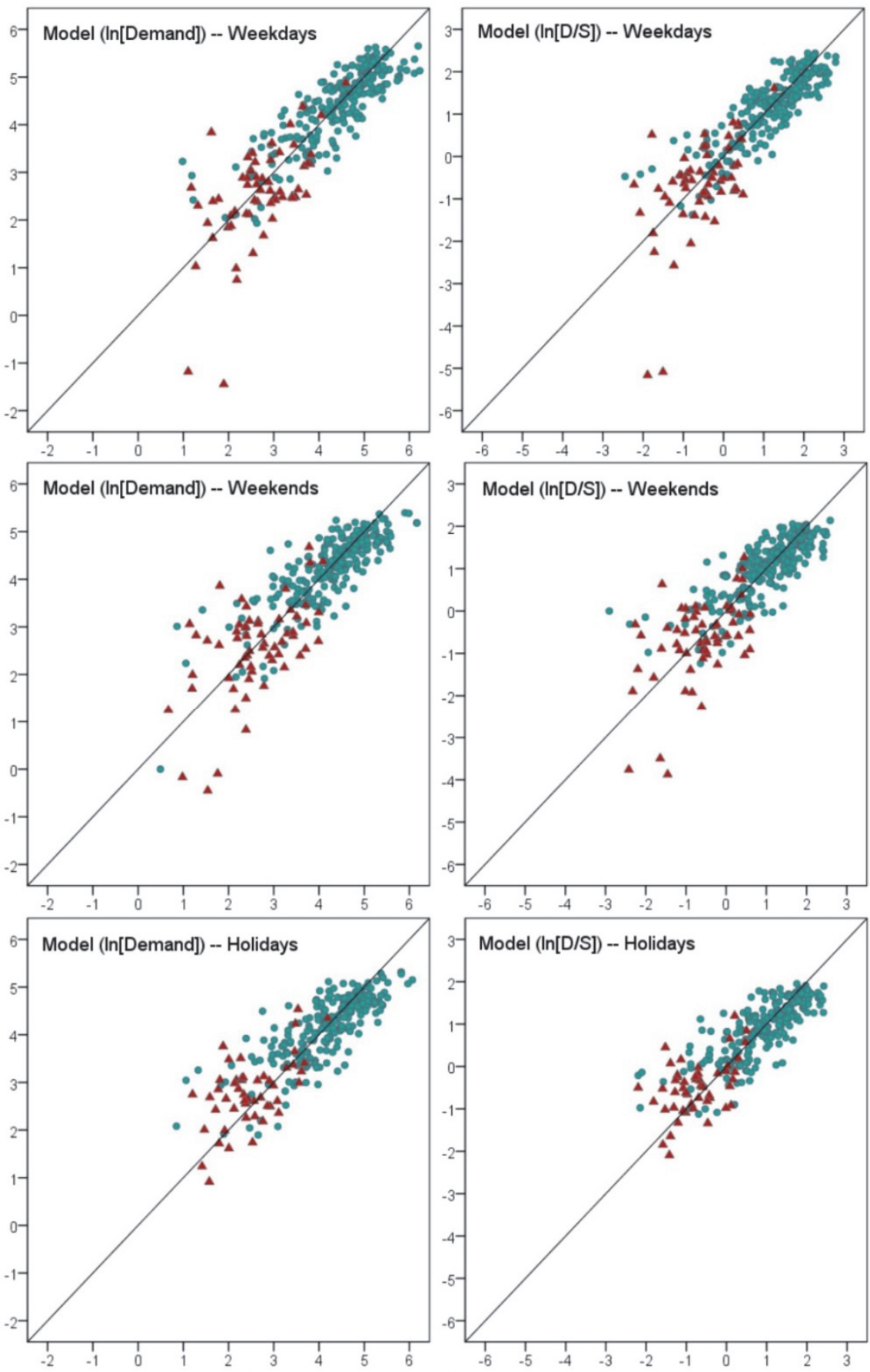

(1) $X$ axis represents the observed value: In[Demand](left column), In[D/S](right column). $Y$ axis represents the model value (2) Blue (circle

Figure 9 Predicted $(Y)$ versus observed $(X)$ values at each station. 

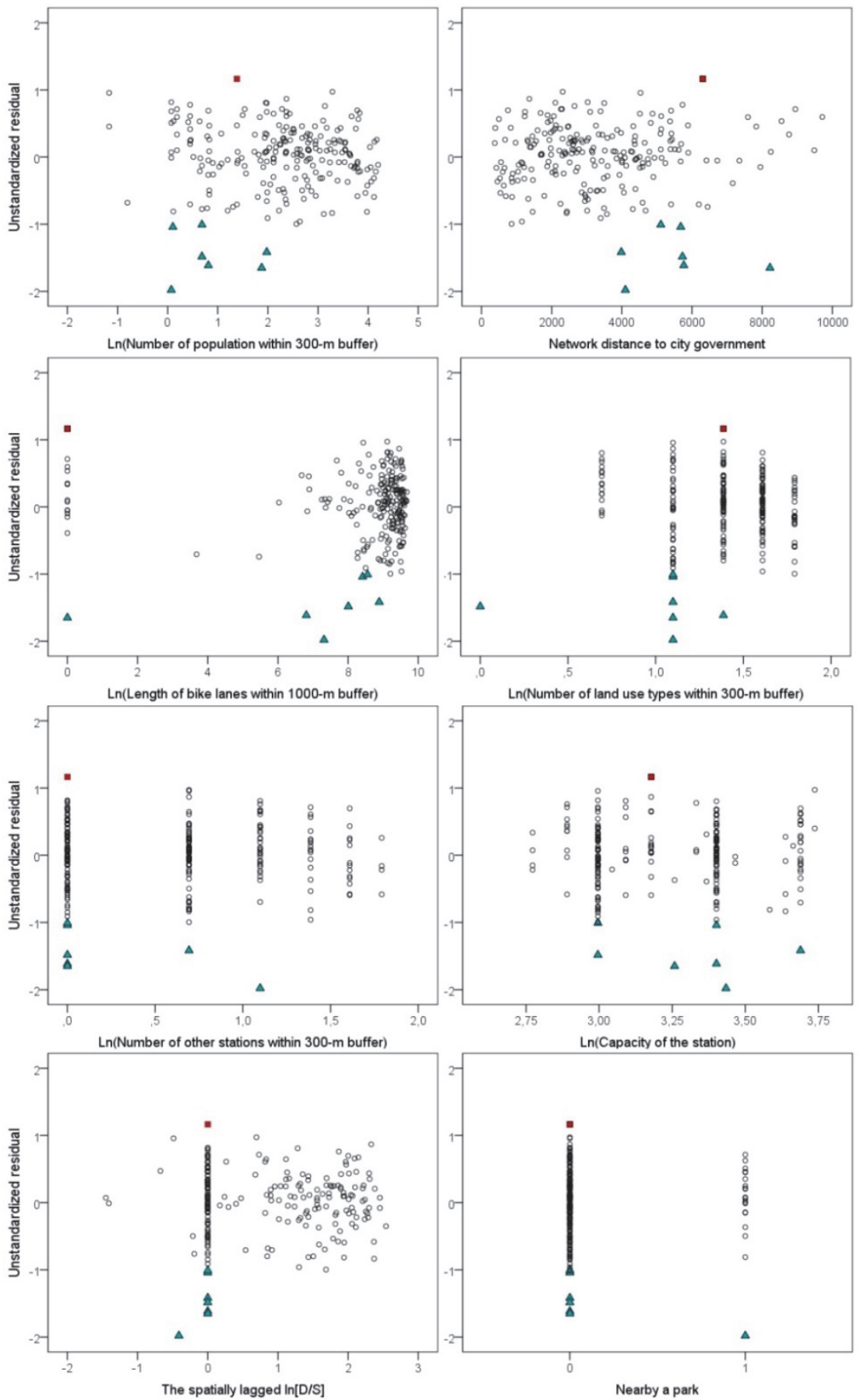

Figure 10 Unstandardized residual versus each significant independent variable at each station (Model In[D/S] of weekdays). 


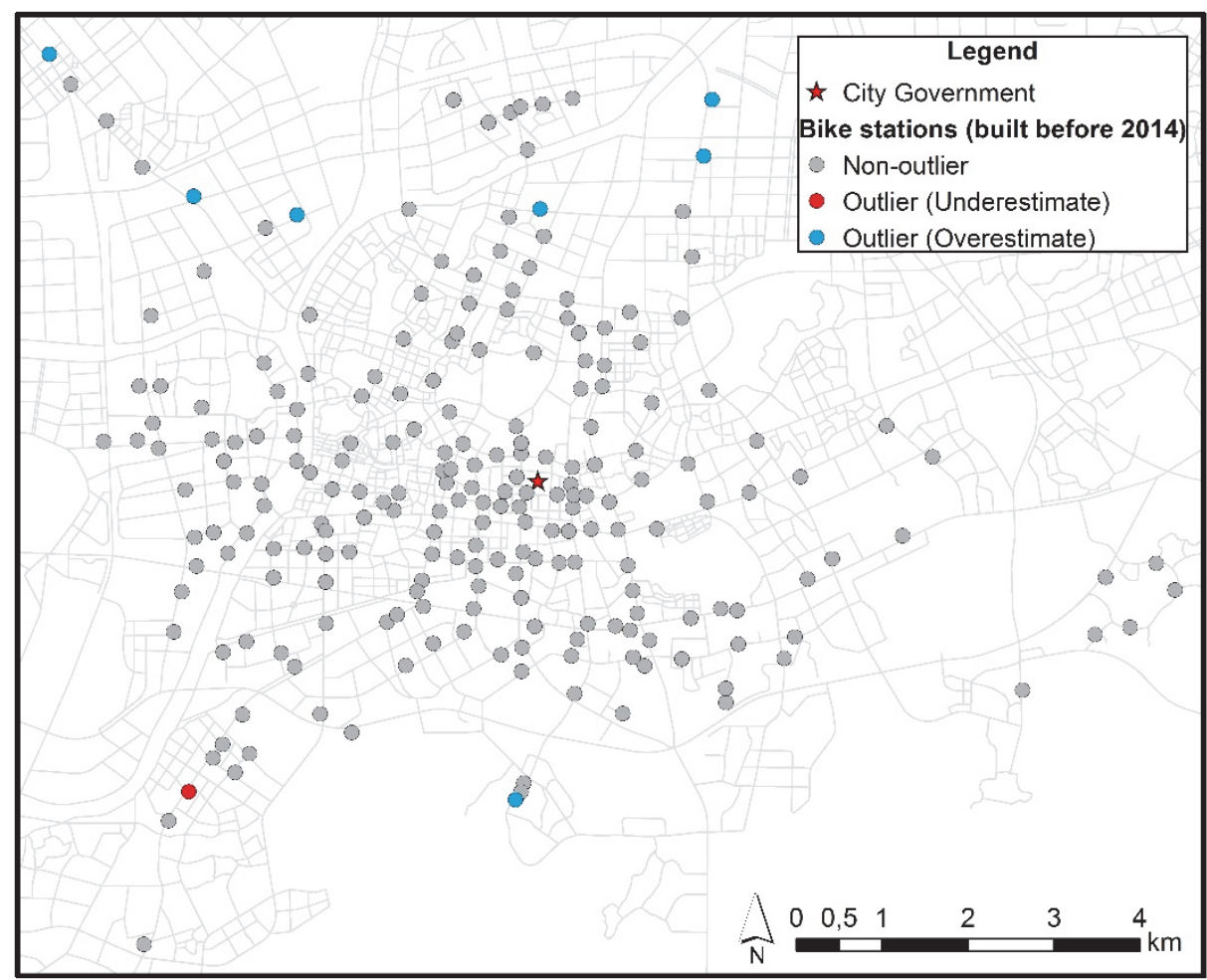

Figure 11 Spatial distribution of outliers and non-outlier (bike stations).

\subsection{Conclusions}

This study employed spatial multiple linear regression analysis to examine the impact of built environmental variables on trip demand as well as demand to supply ratio (D/S) at bike stations. We also considered the spatial spillover effect of nearby stations, i.e. the BSS usage (demand and D/S) and parking slots at nearby stations, using the spatial weighted matrix W. The built environmental variables contain a range of factors relating to station attributes (station capacity, number of nearby stations), accessibility to city centre and population density, cycling infrastructure, public transport variables, and land use characteristics surrounding the bike stations. Spatial variables were computed within the catchment area of each station, and a distance decay function was used to compute the size of population covered by each station. Trip data for Zhongshan's bike-sharing system from February to June 2014 were gathered for carrying out the analysis.

Generally, we found that stations which are closer to the city centre and have a higher population density within the $300 \mathrm{~m}$ buffer generated larger demands and $\mathrm{D} / \mathrm{S}$, which is a common finding (e.g. Daddio (2012)). As expected, users prefer to choose stations that cover more bike lanes and branch roads that 
offer a bike-friendly environment and are more accessible to local communities (same to the findings of Faghih-Imani et al. (2014)). Station capacity shows a positive impact on both daily and hourly demand at the station, implying that users show a preference for stations with a large capacity, in order to increase the chance of finding a bike or parking slot (El-Assi et al., 2015). Although the study area has mixed land use patterns, model results indicated that the larger the number of different land use types within the $300 \mathrm{~m}$ buffer, the larger demand (and D/S) generated at stations. This suggests that more diverse land use types might attract a larger number of users with different travel purposes than a single land use type. Moreover, a larger turnover generated at stations nearby a residential community during weekends and off-peak of weekdays, and lower turnover and demand generated at stations nearby a park during morning and evening peaks of weekdays.

There might be competition between nearby stations, i.e. users shift from a station to a nearby station to pick up or drop off bikes, as the number of other stations within the $300 \mathrm{~m}$ buffer of a station negatively affects both demand and $\mathrm{D} / \mathrm{S}$ at the bike station. Moreover, the spatially lagged dependent variable also indicated the spatial correlation of BSS usage between nearby stations, i.e. demand at a station is positively correlated with demand from nearby stations. This can be explained by two potential reasons: first, nearby station share the same built environment factors that result in the high (or low) demand at these stations (Cervero et al., 2009); second, the spillover effect of demand at nearby stations, i.e. if a station is near its capacity (no available bikes or parking slots), users will shift from a station to nearby stations to pick up or drop off bikes (Rudloff and Lackner, 2014).

The model of demand-supply ratio during weekdays indicated the negative impact of station capacity is 1.7 times larger than the negative impact of the number of other stations within the $300 \mathrm{~m}$ buffer. When keeping other variables constant, we found that adding a new station (with empty capacity) within a $300 \mathrm{~m}$ buffer of a current station to share the capacity of the current station can improve the $\mathrm{D} / \mathrm{S}$ at the current station, i.e. relocating the capacity of a station to a new station within the $300 \mathrm{~m}$ buffer of the station. This suggests that increasing the density of stations with small or medium-sized capacity can enhance the turnover at stations, which will also extend the service area of bike stations and reduce the travel distances.

In our study, the exiting public transport facility does not show a significant influence on the BSS usage at stations, implying that users did not tend to transfer from public bikes to public bus stops (or the other way round) in general, which is quite different from some other studies (e.g. BachandMarleau et al. (2012)). This can mainly be attributed to the local modal split and less attractive public bus system, as well as to the fact that the use of 
public bikes is free in the first hour, which is much cheaper than one trip on a public bus. This suggests that the significant role of the bike-sharing system is not an intuitive feeder mode to exiting public transport system in our study, but serves as a single mode for users to complete the entire trips (similar to the usage of BSS in Zhuzhou city, a medium-sized Chinese city, indicated by Zhang et al. (2015)). Other studies also indicated that the role of bike-sharing systems (e.g. feeder or replacement) varies in different Chinese cities, and bike-sharing systems draw most of its users from unsheltered modes (walking, e-bike, private bike, motorbike) (Campbell et al., 2016; Shaheen et al., 2011; Zhang et al., 2015). Moreover, even in large cities, such as Beijing, Hangzhou, with well-developed public transport systems, public bikes acted as both a supplement and a competitor to existing public transport systems (Campbell et al., 2016; Shaheen et al., 2011). Although the guideline on building public systems is quite the same all over the world at the present stage, there is a substantial necessity to understand the local travel modes before building bikesharing systems, and define the role of bike-sharing systems in the context of cities rather than a priori treat such systems as a feeder mode to exiting public transport systems, so that the system can be used efficiently. 


\section{Chapter 4}

\section{The dynamics of the bike-sharing system ${ }^{3}$}

3 This chapter is based on Zhang, Y., Thomas, T., Brussel, M. J. G., van Maarseveen, M. F. A. M. (2016). Expanding Bicycle-Sharing Systems: Lessons Learnt from an Analysis of Usage. PLoS ONE, 11(12), e0168604. 


\begin{abstract}
Bike-sharing programs, with initiatives to increase bike use and improve accessibility of urban transit, have received increasing attention in growing number of cities across the world. The latest generation of bike-sharing systems has employed smart card technology that produces station-based data or trip-level data. This facilitates the studies of the practical use of these systems. However, few studies have paid attention to the changes in users and system usage over the years, as well as the impact of system expansion on its usage. Monitoring the changes of system usage over years enables the identification of system performance and can serve as an input for improving the location-allocation of stations. The objective of this study is to explore the impact of the expansion of a bicycle-sharing system on the usage of the system. This was conducted for a bicycle-sharing system in Zhongshan (China), using operational usage data of different years following system expansion. To this end, we performed statistical and spatial analyses to examine the changes in both users and system usage between before and after the system expansion. The findings show that there is a big variation in users and aggregate usage following the system expansion. However, the trend in spatial distribution of demand shows no substantial difference over the years, i.e. the same highdemand and low-demand areas appear. There are decreases in demand for some old stations over the years, which can be attributed to either the negative performance of the system or the competition of nearby new stations. Expanding the system not only extends the original users' ability to reach new areas but also attracts new users to use bike-sharing systems. In the conclusions, we present and discuss the findings, and offer recommendations for the further expansion of system.
\end{abstract}

Keywords: bicycle-sharing systems, system expansion, shared-bike use 


\subsection{Introduction}

Cycling is widely associated with benefits in terms of the environment, society, and economy (Krizek et al., 2009; Pucher et al., 2010). The combined use of a bicycle and public transport for a trip, which has been regarded as part of the solution for achieving a more sustainable transport, has grown over the past few years (Keijer and Rietveld 2000; Martens, 2007). Recently, bicyclesharing programs, with initiatives to increase bike use and improve "the last mile" of urban transit, have received increasing attention in more and more cities across the world (DeMaio, 2009; Shaheen et al., 2010). Published studies have shown that for both utilitarian and recreational purposes, cycling has increased in some cities that are operating bicycle-sharing systems (Fishman et al., 2013; O'Brien et al., 2014). Currently, more than 600 such systems are operating around the world, and many systems are being planned and will start operation in the near future (ITDP-China, 2015).

The latest generation of bicycle-sharing systems has employed smart card technology, which enables users to monitor the number of available bikes and parking slots via real-time online maps or mobile apps (O'Brien et al., 2014; Shaheen et al., 2010). This technology produces station-based data or triplevel data, which facilitates studies of the practical use of bicycle-sharing systems (M. Vogel et al., 2014). Some studies have employed data mining techniques (Borgnat et al., 2011; Froehlich et al., 2009; Kaltenbrunner et al., 2010; P. Vogel et al., 2011) and visualization techniques (Beecham and Wood, 2014; Zhao et al., 2015; Zhou, 2015) to uncover the spatial and temporal patterns of cycle trips. Other studies have explored bike-sharing use, in terms of its impact on other transport (Fuller et al., 2012; Jäppinen et al., 2013; Jensen et al., 2010), user demographics (Goodman and Cheshire, 2014; Lathia et al., 2012), and the influence of built environment factors (Buck and Buehler, 2012; Daddio, 2012; El-Assi et al., 2015; Faghih-Imani and Eluru, 2015, 2016; Faghih-Imani et al., 2014; Gonzalez et al., 2016; Nair et al., 2013; Rixey, 2013; Wang et al., 2016), weather and calendar events (Corcoran et al., 2014; Gebhart and Noland, 2014) on shared bike demand. Most of the aforementioned studies, except one from Goodman and Cheshire (2014), did not address the changes in usage (i.e. in terms of both users and demand) over the years, and did not study the impact of the system expansion on its performance. However, system usage might not be stationary, and may change over the years. Examining changes in usage of a system over the years enables the identification of factors that influence the system's performance, and can also serve as an input for improving the location-allocation of stations and planning for new stations.

In this context, the objective of this study is to explore the changes of system usage over the years and impact of the expansion of a bicycle-sharing system on the usage of the system. This study was conducted for a bicycle-sharing 
system in Zhongshan (China), using trip data from March 2012, March 2013, and March 2014. Such a system gradually expanded the number of stations equipped with parking slots between March 2012 and March 2013, and again between March 2013 and March 2014. This study contributes to the understanding of how the system usage (in terms of both users and demand) changes following the expansion of the system. Moreover, this study also provides insights into: (1) what information can be extracted from trip data to evaluate the impact of system expansion on its use; and (2) what can be learnt from this evaluation to promote the further extension of the system.

The remainder of this paper is organized as follows. The next section provides a literature review. Section 4.3 introduces the study area and data preparation, and explains the research method. Section 4.4 presents and discusses the results. Finally, Section 4.5 draws the key conclusions and offers the recommendations for the system expansion and further study.

\subsection{Literature review}

With the availability of open data, i.e. station-based data or trip level data, a large number of studies have been carried out to explore the practical usage of bicycle-sharing systems. In general, those studies mainly cover four aspects: Firstly, to explore the spatial and temporal patterns of bike use over the time of day, using data mining (Froehlich et al., 2009; Kaltenbrunner et al., 2010; P. Vogel et al., 2011) and visualization (Beecham and Wood, 2014; Zhao et al., 2015; Zhou, 2015) techniques. Froehlich et al. (Froehlich et al., 2009) grouped stations based on bicycle activity at the stations of Barcelona's public bike system, and Kaltenbrunner et al. (Kaltenbrunner et al., 2010) extended the former analysis by predicting bicycle activity at Barcelona's stations over the hours of the day. Vogel et al. (P. Vogel et al., 2011) examined activity patterns of bike use at the stations of Vienna's system. They generally found that usage during peak hours of weekdays are quite different from that of weekends, and that differences in peak usage at stations might be associated with the kind of activities in the neighbourhood. Beecham et al. (Beecham and Wood, 2014) analysed cycling trips by members of London's bike-sharing system. They found that women tend to use public bikes at weekends and within London's parks, while men tend to use public bikes for commuting. Moreover, women's trips are highly spatially structured and mainly occur in areas with cycle routes and/or with slower traffic. Similar visual techniques were employed by Zhao et al. (Zhao et al., 2015), who analysed the cycling trip chains by gender and day of the week in Nanjing, China. They found that on weekdays, women tend to make multiple-circle trips and spend more time on cycling than men. Moreover, Zhou (2015) investigated the spatial-temporal pattern of cycling trips of the Chicago bike-sharing system, and uncovered different travel patterns between weekdays and weekends as well as between customers and subscribers. 
Secondly, to study the characteristics of the usage of bicycle-sharing systems, either for a single system or in a comparison of different systems. Jensen et al. (Jensen et al., 2010) found that public bikes compete with the car in terms of speed in downtown Lyon by analysing 11.6 million bicycle-sharing trips. Based on station data, Jäppinen et al. (Jäppinen et al., 2013) indicated that integration of public bikes with traditional public transportation can promote sustainable daily mobility in Helsinki. Studies on London's bicycle-sharing systems found that two strikes of the London subway led to an increase of the number and duration of public bike trips (Fuller et al., 2012), and that easier access to the system can promote weekday commuting and weekend use (Lathia et al., 2012). Goodman and Cheshire (Goodman and Cheshire, 2014) found that the introduction of casual access to London's system encouraged more women to use the system, and the extension of the system to highlydeprived areas not only attracts new users but also increases local travel in such areas. O'Brien et al. (O'Brien et al., 2014) examined the usage of 38 global bicycle-sharing systems, and indicated that Asian systems have a lower compactness than European/Middle Eastern systems. They could also group Chinese systems together based on system attributes (e.g. system size, daily usage, etc.). Zhao et al. (Zhao et al., 2014) compared 69 Chinese bike-sharing systems. Based on the effects of urban population, government expenditure, system size, and operation policy on daily use and daily use per bike, they suggested that the bike-member ratio could be less than 0.2 and that the adoption of personal credit and universal cards to access to systems influences the usage in a positive way.

Thirdly, to examine the impact of built environment factors and weather conditions on the demand at stations. In general, some studies found that population and job density, proximity to transit stations (metro and public bus stations) and bike lanes, and points of interests (retail shops, parks, restaurants, etc.) within the service area are positively associated with ridership at stations (Buck and Buehler, 2012; Daddio, 2012; El-Assi et al., 2015; Faghih-Imani and Eluru, 2015, 2016; Faghih-Imani et al., 2014; Gonzalez et al., 2016; Nair et al., 2013; Rixey, 2013; Wang et al., 2016). Moreover, station size and number of bike stations within the catchment area also have an impact on the bike-sharing demand at stations (El-Assi et al., 2015; Faghih-Imani and Eluru, 2015; Faghih-Imani et al., 2014). Severe weather conditions are associated with a negative impact on the system usage (Corcoran et al., 2014; Gebhart and Noland, 2014). Finally, a small number of studies focuses on proposing a mathematical algorithm to deal with bikesharing rebalancing problem (Dell'Amico et al., 2016; Kadri et al., 2016; Neumann-Saavedra et al., 2015).

Most of aforementioned studies, however, do not look at the dynamics of bikesharing systems. Changes over time do not only occur in demand, but possibly 
also in the (type of) users. Do users and their demand change over time? This paper explores these questions in order to better understand the system and its future potential. It also investigates changes in usage over the years to identify which factors influence the system's performance. This may provide useful insight for improving the location-allocation of current stations and for planning new stations. This study was conducted for a bicycle-sharing system in Zhongshan (China), using trip data from March 2012, March 2013, and March 2014. The system gradually expanded the number of stations equipped with parking slots between March 2012 and March 2013, and again between March 2013 and March 2014. To this end, we examined the changes in both users (UserID) and the system usage by comparing March 2012 with March 2013, and comparing March 2013 with March 2014. In this study, we consider the changes in the system as a whole as well as in the spatial distribution of demand before and after the system expansion.

\subsection{Context and Methods}

\subsubsection{Study area}

Zhongshan city is a medium-sized city that is located in the Guangdong province of China, and directly opposite Hong Kong. The city is a prefecturelevel city (Figure 12 (A)) whose government directly administers six districts corresponding to the urban area, and eighteen towns (in China, town is an administrative unit, into which counties and districts are divided). Among these, four districts - the Xi, Shiqi, Dong, and Nan districts - constitute the "major urban area" (Figure 12 (B), which covers an area of $170 \mathrm{~km} 2$ and was home to a population of around 530,000 in 2013(ZhongshanStatisticsBureau, 2014). This major urban area can be characterized by a high population density and a concentration of residence, employment, shopping, entertainment, culture, and political power. In addition, the eastern and southern urban areas are the Torch $\mathrm{Hi}$-tech Industrial Development district $(90 \mathrm{~km} 2)$ and the Wuguishan district $(113 \mathrm{~km} 2)$ respectively. The former is a national-level hi-tech industrial development zone with a population of 240,000 in 2013 , and the latter is mainly intended for tourism and agriculture with a population of 48,000 in 2013. 


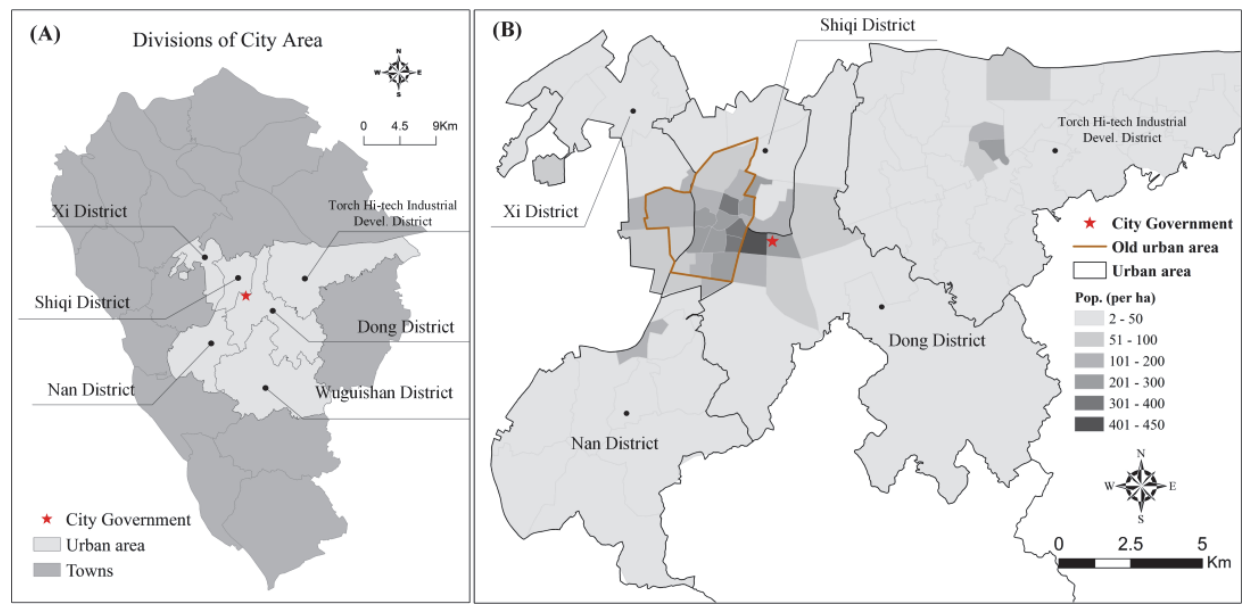

(A) Location and Divisions of study area; (B) Population density distributed in the study area.

Figure 12 Study area.

According to travel statistics from Zhongshan transport planning department (this was done before running the bike-sharing program), non-motorized modes account for $46.3 \%$ of total trips, of which $24.3 \%$ are walking trips. The shares of motorcycle and private car trips are $39.8 \%$ and $8.5 \%$ respectively, whereas public bus trips only account for $4.2 \%$. The average trip lengths in the major urban area are $0.8 \mathrm{~km}, 2.8 \mathrm{~km}$, and $4.8 \mathrm{~km}$ for walking, cycling (bike and e-bike), and public bus trips respectively. In addition, $94.8 \%$ of all trips lasted less than 30 mins. In conclusion, non-motorized (walking and cycling) and motorcycle modes are the main travel modes in the "major urban area" while public transport is not very attractive to most residents.

\subsubsection{Zhongshan's bicycle-sharing system and data preparation}

Zhongshan's bicycle-sharing system was launched in October 2011 and is a 24/7 self-service system. Users can pick up and return public bikes at any station during the day, using a smart card that has a unique User-ID. Each user can apply for a smart card by registering as a member and depositing 200CNY. For each trip, the first hour is free, and any extra hours are charged at an incremental price (1CNY per hour), which is much cheaper than a trip by local public bus (2 CNY per trip).

In the urban area, there were 180 bike stations equipped with 4530 parking slots in March 2012, increasing to 224 stations with 5959 parking slots in March 2013, and then further expanding to 245 stations equipped with 6547 parking slots in March 2014. The stations are shown in Fig 2, we use the label "station12" for stations that were built before March 2012, label "station13" for stations that were built between March 2012 and March 2013, and label 
"station14" for stations built after March 2013. The average number of parking slots per station is 25, 32, 28 for ""station12", "station13", and "station14" respectively. Figure $13(\mathrm{~A})$ shows how the system gradually expanded from the city centre to the outskirts. It also shows that the density of stations is highest in the central area which has the highest population density and includes the city government. Figure 13 (B) shows the size of the stations. It is worth mentioning that the majority of newly-built stations have quite a high capacity.

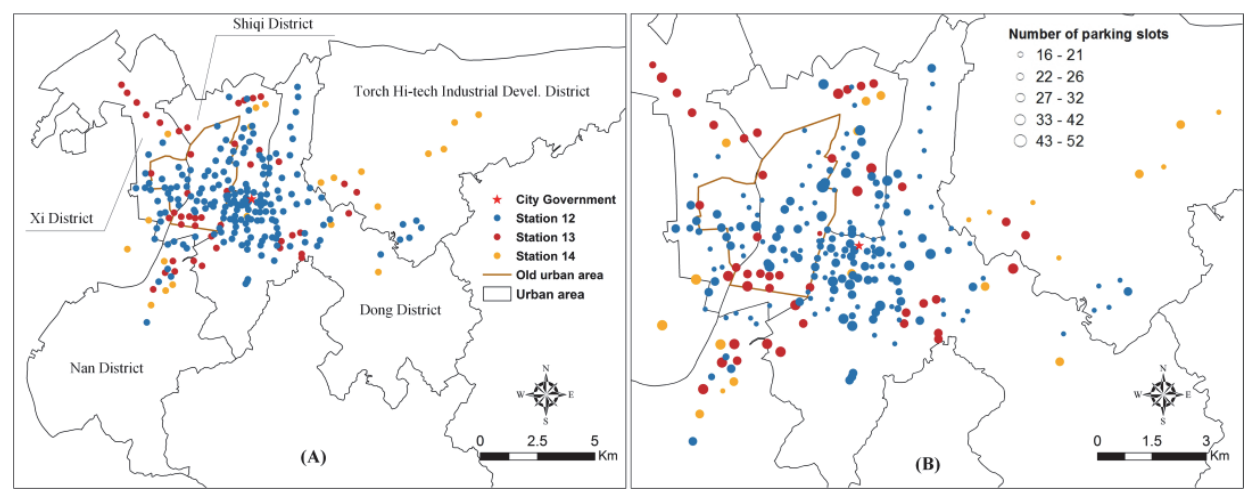

(A) spatial distribution of stations that were built before and after the system expansion. (B) The number of parking slots at each of bike stations.

Figure 13 The spatial distribution of bike stations and capacities in the study area.

The data were collected from the Transport Department of the Urban Planning and Design Institute of Zhongshan (China). The provided trip data are from March 2012, March 2013, and March 2014, and include User-ID, pickup and return stations, and pickup time and return time. The duration of each trip is calculated by subtracting the pickup time from the return time. Based on data screening, we excluded two types of inaccurate records from the original trip database: (1) trips for which pickup or return information was missing; and (2) trips that lasted less than 1 minute, for which we assume no trip was actually made. As a result, we acquired data for 473,236 trips in March 2012, 453,846 trips in March 2013 and 398,305 trips in March 2014.

Weather conditions were considered as one of the potential factors that could have affected the bike use, but only extreme weather conditions (pouring rain or blistering heat) seem to really discourage cycling (Frade and Ribeiro, 2014). Zhongshan has a subtropical climate with an average temperature of $22^{\circ} \mathrm{C}$ and, in March, the weather is warm without strong winds. Rainfall was not extreme either and did not appear to have a significant influence on daily bike use. According to the statistical correlation between daily amount of rainfall (the whole day, as well as different time periods) and daily trips, the number 
of daily trips was not significantly $(p<0.05)$ influenced by daily rainfall. We therefore did not consider weather conditions in the further analysis.

\subsubsection{Methods}

This study aims to explore how the usage of the system changes following system expansion. To this end, we performed both statistical and spatial analyses to examine the changes in both users and system usage between March 2012 and March 2013, and between March 2013 and March 2014. The analyses were carried out using SPSS and ArcGIS. We separate travel on weekdays from weekends and also distinguish between morning peak hours and evening peak hours.

Comparing "User-IDs" before and after the system expansion, users are divided into three groups: (1) former users who used the system before the system expansion but not at all after the system expansion; (2) steady users who used the system both before and after the system expansion; and (3) new users who started to use public bikes only after the system expansion.

The system usage was investigated by: (1) the aggregate use of the system and (2) the spatial distribution of both users' demand and the ratio of demand to supply $(D / S)$. We examined the system usage for both all users and per user group. The aggregate use of the system is based on daily usage (distinguishing weekdays and weekends) and hourly usage (distinguishing morning peak hours and evening peak hours). The definition of morning peak hours and evening peak hours is based on the number of trips generated over the hour of day. Morning peak hours are 7:00-9:00 on weekdays and 8:00-9:00 on weekends, and evening peak hours are 17:00-19:00 on both weekdays and weekends. Daily and hourly usage were described by the usage metrics which mainly include the average number of users, average number of trips, average number of trips per user, average number of demands per station (distinguishing between "old" stations and newly-built stations), average trip length, and average trip duration. The number of trips corresponds with the demand for bikes, as one trip means a user picks up a bike from a station and returns the bike to another or the same station. The demand at each station was calculated by the sum of departure trips (i.e. picking up bikes) and arrival trips (i.e. returning bikes) at the station, as the number of pick-ups is comparable to the number of returns at each station. We decided to use the "Median" to calculate the "average" value of aforementioned usage metrics, which can mitigate the impact of some outliers (e.g. sharp decrease) on the measure of daily use.

The spatially oriented approach provides operators and researchers with a better understanding of usage and user patterns (Corcoran and $\mathrm{Li}, 2014$ ). The spatial distribution of both demand and $D / S$ was used to uncover the trend in 
distribution of bike-sharing use across the urban area. Moreover, the D/S can be an indication of the relationship between users' demand and system's supply. The users' demand refers to the average number of trips generated by a group of users, which is a metric of the aggregate use of the system. The system's supply refers to the number of parking slots, which was not a constant and increased after the system expansion.

The spatial distribution was visualized by a spatial fishnet that divided the urban area into a bunch of grid cells. The spatial fishnet was created in ArcGIS, with each cell having a size of 50 by 50 meters. Figure 14 shows how we computed the weight of each cell, which determines the relative importance of each cell, and lays a foundation for smoothing the overall users' demand and system's supply over grid cells.

The equations 4.1 and 4.2 show how we smoothed the demand (left column) and supply (right column) at stations (discrete locations) over the cells. We first created a catchment area (300m radius) consisting of six 50-m concentric bands, around each bike station (see P1 in Figure14). The size of the catchment area was chosen such that it is approximately equal to $344 \mathrm{~m}$ average distance between neighbouring stations. This catchment area also corresponds with a suitable walking distance. The catchment areas were generated based on network distances (the shape of each catchment area is regular or irregular polygon depending on the road network), which are considered for walking to transit facilities. We then redistributed the station's demand and supply (i.e. slots) to each band based on distance decay (Eq. 4.1), that is, $S D_{b}$ and $S S_{b}$. This decay actually represents the distribution of the users' actual origins or destinations around each station. In other words, users are more likely to use stations when they are very nearby, but they can still use a station if they have to walk some distance. Afterwards we carried out a spatial analysis to intersect catchment area (bands) with spatial fishnet (grid cells) to distribute the bands' demand $\left(S D_{b}\right)$ and supply $\left(S S_{b}\right)$ to each grid cell (Eq. 4.2). Further, Eq. 4.3 shows how we assigned a weight of demand $\left(W D_{C}\right)$ and a weight of supply $\left(W S_{C}\right)$ to each grid cell and the sum of each cell's $W D_{C}$ (and $W S_{C}$ ) is 1.

The Eq. 4.4, $D_{c}$ and $S_{c}$, represent the number of users'demand and system's supply respectively, which is given to each cell. For example, the spatial distribution of demand and the spatial distribution of D/S (the ratio of $D_{c}$ to $S_{c}$ ) over the cells, are shown in Figure 16 and Figure 19 respectively.

In the results section, we describe the trends in spatial distribution of users' demand and D/S, and employ Hot Spot analysis (spatial statistics in ArcGIS) to identify statistically significant hot spots and cold spots for users' demand using Getis-Ord Gi* statistic. This may uncover whether there are significant differences in the spatial distribution of users' demands following the system 
expansion. We also examine the differences in number of demands between user groups over the grid cells. However, there is a considerable difference in total demand between the different groups. To take this difference into account, we normalized the demand of user groups. As an example, Eq. 4.5 shows how we calculated the difference in spatial demand (i.e. $D_{c}$ ) between U12 and U13, which is users in 2012 and users in 2013. In Eq. 4.5, the factor $\alpha$ is the ratio of overall demand between $\mathrm{U} 12$ and $\mathrm{U} 13$. The function of $N D_{c(U 12)}$ is used to normalize the cells' demand of U12, and consequently the sum of $N D_{c(U 12)}$ is equal to the sum of $D_{c(U 13)}$. The function of $N D_{(13 v s 12)}$ calculates the normalized difference in each cell's demand between U12 and U13. As a result, the values of $N D_{(13 v s 12)}$ of all cells are normally distributed with a mean 0 and a sum 0 ; we therefore use standard deviation as a unit to visualize the difference in demand over grid cells in Figure 21 and Figure 22. 


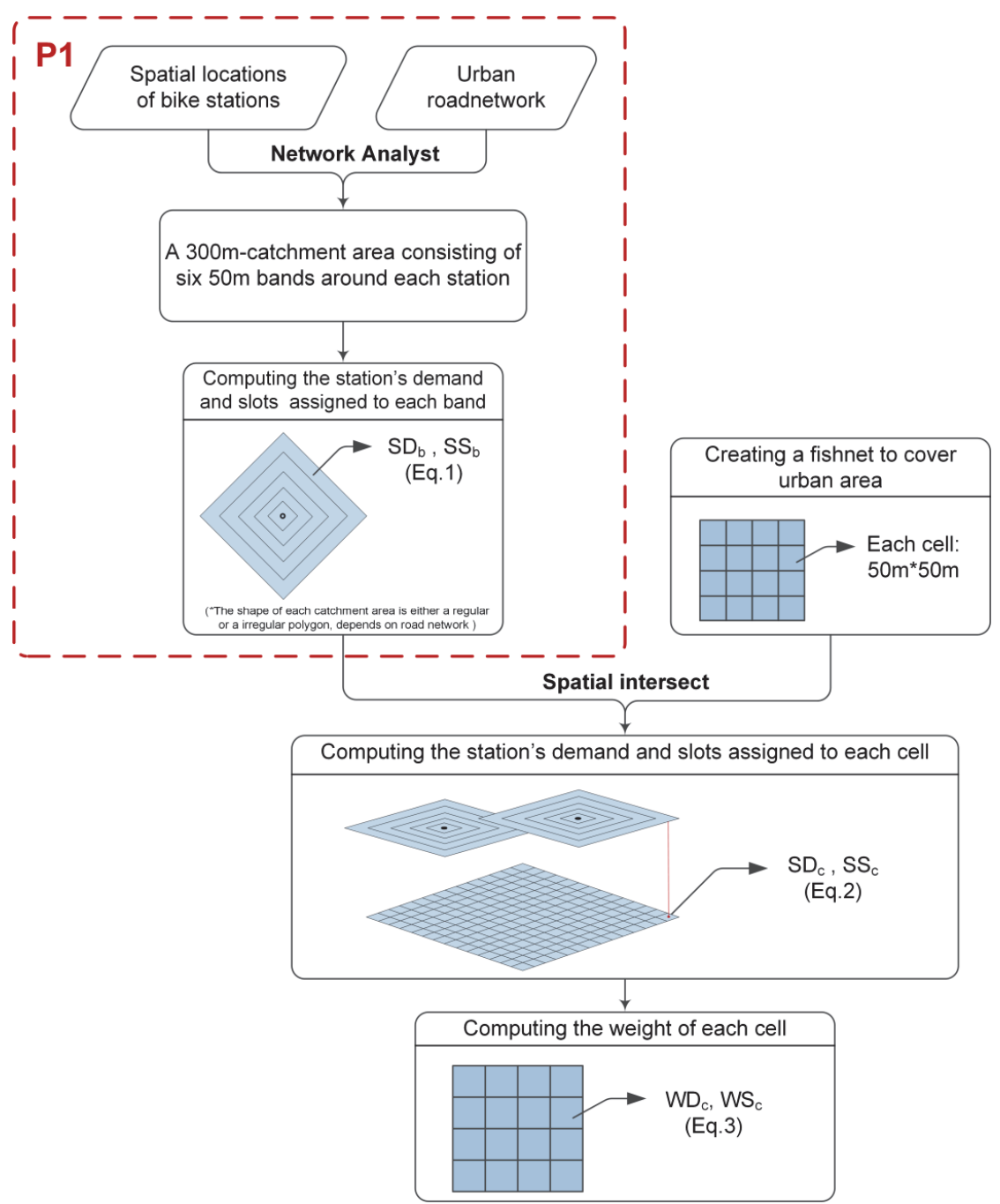

Figure 14 A diagram of computing the weight of each cell.

$$
\begin{array}{ll}
S D_{b}=D_{i, j}=D_{i}^{2} /\left(d_{j} * \sum_{j} D_{i} / d_{j}\right) & S S_{b}=S_{i, j}=S_{i}^{2} /\left(d_{j} * \sum_{j} S_{i} / d_{j}\right) \\
S D_{C}=\sum_{b=1}^{n} S D_{b} * a p_{b} & S S_{c}=\sum_{b=1}^{n} S S_{b} * a p_{b} \\
W D_{C}=S D_{c} / \sum_{c}^{n} S D_{c} & W S_{C}=S S_{c} / \sum_{c}^{n} S S_{c} \\
D_{c}=W D_{c} * D_{\text {user }} & S_{c}=W D_{s} * S_{\text {system }}
\end{array}
$$




$$
\begin{array}{lll}
N D_{(13 v s 12)}=D_{c(U 13)}-N D_{c(U 12)} & N D_{c(U 12)}=D_{c(U 12)} / \alpha & \text { Eq. } 4.5
\end{array}
$$

Where:

- $\quad b$ is the ID of each band $(b=1, \ldots n)$, and $i$ is the ID of each bike station;

- $d_{j}$ is the distance of the band, $d_{j}=50 \mathrm{~m}, 100 \mathrm{~m}, 150 \mathrm{~m}, 200 \mathrm{~m}, 250 \mathrm{~m}, 300 \mathrm{~m}$, in which $j=1,2,3$... 6 respectively;

- $\quad D_{i}$ is the number of demands of station $i ; S_{i}$ is the number of parking slots of station $i$;

- $\quad a p_{b}$ is the area proportion of each cell that spatially overlaid with distance band $b$;

- $\quad D_{\text {user }}$ is the average daily trips generated by a user-group;

- $\quad S_{\text {system }}$ indicates the amount of parking slots.

\subsection{Results and Discussions}

In this section, we present and discuss the results of two aspects. Section 4.4.1 presents the aggregate use of the system by different user groups before and after the system expansion. Section 4.4.2 presents the trends and the changes in spatial demand by users between before and after the system expansion. Travel on weekdays was analysed separately from travel on weekends and we also distinguish between morning peak hours and evening peak hours.

\subsubsection{Aggregate use of the system before and after system expansion}

Table 10 describes the aggregate daily and hourly use of the system by all users, on weekdays and weekends of March 2012, March 2013, and March 2014. It reveals there is an overall decrease in daily use between 2012 and 2014 , despite the expansion of the system. This decrease is most distinct in weekends. Not only does the number of users decrease, the average number of trips per user is also declining. The expansion of the system has resulted in extra usage at new stations (shown in the rows "daily demand per station13" and "daily demand per station14" for the station added in 2013 and 2014 respectively). In 2013, this was quite a substantial part of the total, actually resulting in an overall increase in usage for workdays between 2012 and 2013. However, after the second expansion the number of new trips per added station decreased ( 81 for stations added in 2013 and only 25 for stations added in 2014). This shows that stations added in 2014 have less demand than stations added in 2013, which can be attributed to the fact that the majority of "station 14" is on the outskirts. The fact that newly-built stations have not led to an overall increase in demand can be attributed to a significant decline in usage at the original stations (from 182 per station 12 in 2012 to 139 per station 12 in 2014). Partly, this can be explained by the fact that new stations 
might compete with older stations, but the rate of decline is somewhat surprising. To provide a better interpretation of this result, we need to consider different user types, which will be done in Table 11 and Table 12.

In addition, the change in hourly usage during morning peak and evening peak hours is comparable with the change of daily usage following the system expansion. Regarding the comparisons of hourly usage during morning peak hours and evening peak hours, the users' demand (the average number of hourly trips) during evening peak hours is slightly larger than during morning peak hours. This might be attributed to more people use the system (or users generated more trips) during evening peak hours, because people have more leisure activities (or spare time) in the evening (after work) than in the morning. When we look at the demand at stations, there is no considerable difference in hourly demand per station between morning peak hours and evening peak hours, especially after the system expansion. Moreover, Figure 15 describes the comparison of the number of hourly demands at each station between morning peak hours ( $Y$ axis) and evening peak hours ( $X$ axis). This indicates that the number of hourly demands at each station during morning peak is comparable with that during evening peak, especially in March 2013 and March 2014. Figure 15 also indicates that bike stations that have high demand during morning peak hours also generate a high demand during evening peak hours. This implies that the spatial distribution of demand during morning peak hours is similar to that during evening peak hours. Finally, there is no significant difference in trip characteristics between, before and after the system expansion: generally the average trip length and average trip duration are both quite short. 
Table 10 The aggregate use of the system by all users in March 2012, March2013, and March 2014.

\begin{tabular}{|c|c|c|c|c|c|c|}
\hline \multirow[b]{2}{*}{ Usage metrics } & \multicolumn{3}{|l|}{ Weekdays } & \multicolumn{3}{|c|}{ Weekend } \\
\hline & $\begin{array}{l}2012 \\
\text { ( } 22 \text { days) }\end{array}$ & $\begin{array}{l}2013 \\
(21 \\
\text { days) }\end{array}$ & $\begin{array}{l}2014 \\
\text { ( } 21 \\
\text { days) }\end{array}$ & \begin{tabular}{|l}
2012 \\
$(9$ \\
days)
\end{tabular} & $\begin{array}{l}2013 \\
(10 \\
\text { days) }\end{array}$ & $\begin{array}{l}2014 \\
\text { (10 days) }\end{array}$ \\
\hline Daily users ${ }^{a}$ & 9075 & 9429 & 8374 & 8803 & 8328 & 6789 \\
\hline Daily trips & 16292 & 16481 & 14562 & 15570 & 14703 & 11398 \\
\hline Daily trips/user & 1.79 & 1.75 & 1.71 & 1.77 & 1.74 & 1.68 \\
\hline $\begin{array}{l}\text { Daily demand per } \\
\text { station } 12\end{array}$ & 182 & 167 & 139 & 174 & 147 & 109 \\
\hline $\begin{array}{l}\text { Daily demand per } \\
\text { station } 13\end{array}$ & - & 71 & 81 & - & 69 & 65 \\
\hline $\begin{array}{l}\text { Daily demand per } \\
\text { station } 14\end{array}$ & - & - & 25 & - & - & 23 \\
\hline Hourly trips $(\mathrm{MP})^{\mathrm{b}}$ & 1460.75 & 1664.5 & 1523 & 1080 & 1165 & 1106 \\
\hline $\begin{array}{l}\text { Hourly demand per } \\
\text { station } 12 \text { (MP) }\end{array}$ & 13.5 & 13.83 & 11.50 & 9.67 & 8.1 & 7.40 \\
\hline $\begin{array}{l}\text { Hourly demand per } \\
\text { station } 13 \text { (MP) }\end{array}$ & - & 6.67 & 6.93 & - & 3.7 & 4.30 \\
\hline $\begin{array}{l}\text { Hourly demand per } \\
\text { station } 14 \text { (MP) }\end{array}$ & - & - & 1.43 & - & - & 1.30 \\
\hline Hourly trips $(E P)^{c}$ & 1886.5 & 1878 & 1628 & 1552 & 1362.5 & 1122.25 \\
\hline $\begin{array}{l}\text { Hourly demand per } \\
\text { station } 12(\mathrm{EP})\end{array}$ & 15.94 & 13.71 & 11.93 & 12.36 & 9.15 & 7.15 \\
\hline $\begin{array}{l}\text { Hourly trips per } \\
\text { station } 13 \text { (EP) }\end{array}$ & - & 5.76 & 7.74 & - & 4.05 & 4.50 \\
\hline $\begin{array}{l}\text { Hourly trips per } \\
\text { station } 14 \text { (EP) }\end{array}$ & - & - & 1.59 & - & - & 1.15 \\
\hline Trip length (m) & 1356 & 1334 & 1346 & 1334 & 1299 & 1321 \\
\hline Travel time (minutes) & 11 & 10 & 10 & 12 & 11 & 11 \\
\hline
\end{tabular}

a One user represents a User-ID that belongs to a specific person.

$b$ "MP" is the abbreviation for "morning peak hours".

$c$ "EP" is the abbreviation for "evening peak hours".

Users are divided into three groups - former users, steady users, and new users - based on the comparison of User-IDs between, before and after the system expansion. Table 11 and Table 12 describe the division of user groups and the aggregate use of the system by each user-group on weekdays and weekends respectively. For each user group, the number of users on weekdays is higher than that on weekends, demonstrating that some users only used the system on weekdays.

Table 11 describes the aggregate daily and hourly use of the system by each user group, on weekdays and weekends of March 2012, March 2013, and March 2014. It shows that there is a great variation in users. About only half of the users are steady users (when comparing between successive years), while the 
rest are former or new users. This indicates that the system is quite dynamic and has not (yet) found some form of equilibrium. The system is also quite new and still expanding. Interestingly, there are more new users than former users. They use the system more frequently and also make more trips than former users. However, as we have seen, the overall demand has declined over time. This can be attributed to the steady users. These users have used the system less frequently over time (resulting in a decrease of the number of users per day), and also made fewer trips (resulting in a decrease in the number of trips per user per day). These trends are both visible for workdays and weekends. It is not clear why there is a decline in usage among steady users, especially in the light of an expanding system. To provide better interpretation of these results, we investigated the spatial distribution of demand before and after system expansion, which will be presented in the next subsection. 

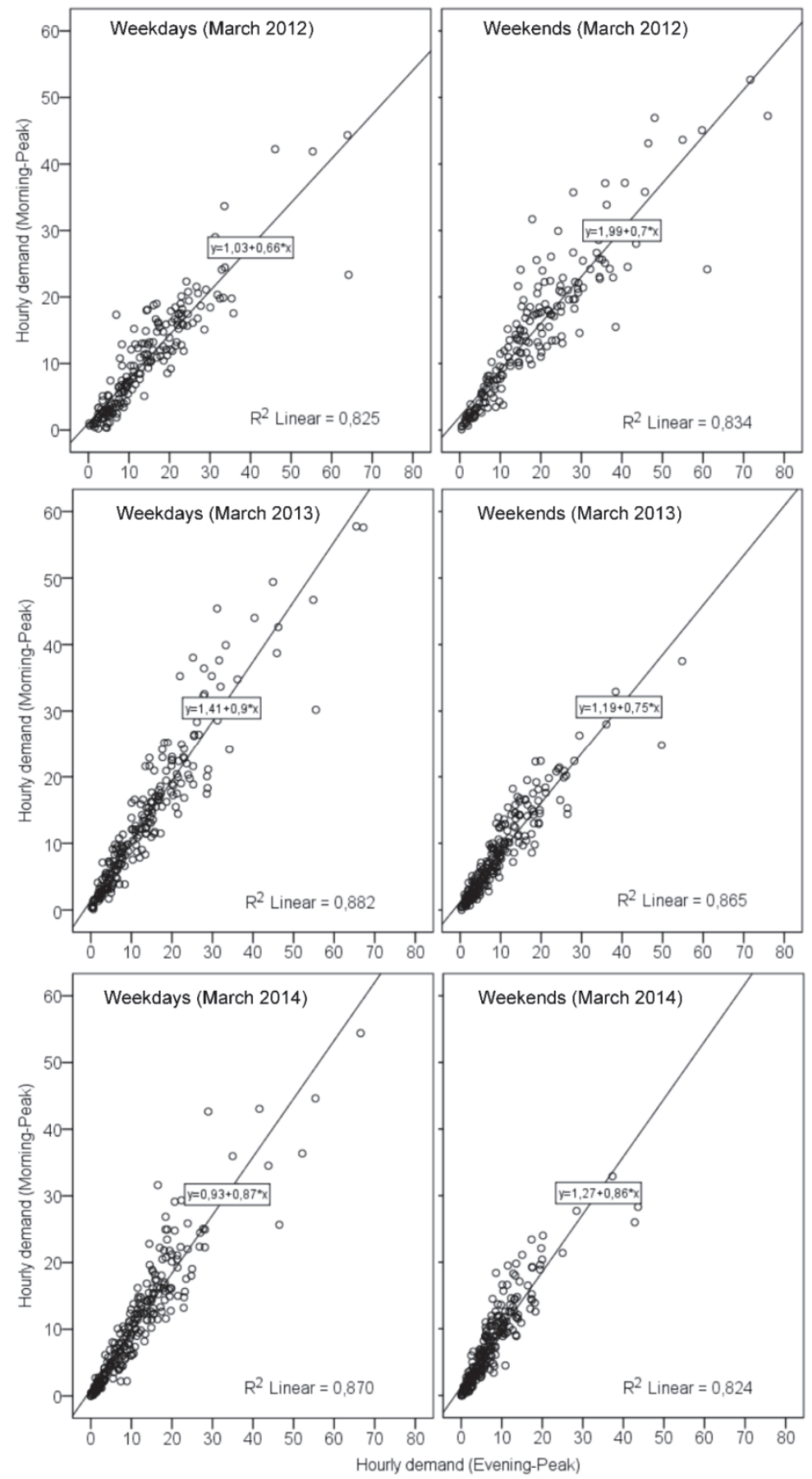

(1) The points represent each of bike stations. (2)Y axis represents the average number of hourly demand during morning peak hours, and $\mathrm{X}$ axis represents the average number of hourly demand during evening peak hours.

Figure 15 Comparisons of hourly demand during morning peak and evening peak hours at stations. 
Table 11 The aggregate use of the system by steady users, former users, and new users on weekdays.

\begin{tabular}{|c|c|c|c|c|c|}
\hline \multirow{2}{*}{\multicolumn{2}{|c|}{ Usage metrics (Median) }} & \multicolumn{2}{|c|}{ Mar 12 vs Mar 13} & \multicolumn{2}{|c|}{ Mar 13 vs Mar 14} \\
\hline & & \multirow{2}{*}{$\frac{2012}{4864}$} & \multirow{2}{*}{$\frac{2013}{4127}$} & \multirow{2}{*}{$\frac{2013}{4888}$} & \multirow{2}{*}{$\frac{2014}{4044}$} \\
\hline \multirow{16}{*}{$\begin{array}{l}\text { Steady } \\
\text { users }\end{array}$} & Daily users & & & & \\
\hline & Daily trips & 8842 & 7024 & 8793 & 6756 \\
\hline & Daily trips/user & 1.8 & 1.69 & 1.78 & 1.66 \\
\hline & $\begin{array}{l}\text { Daily demand per } \\
\text { station } 12\end{array}$ & 99 & 73 & 89 & 66 \\
\hline & $\begin{array}{l}\text { Daily demand per } \\
\text { station } 13\end{array}$ & - & 24 & 36 & 36 \\
\hline & $\begin{array}{l}\text { Daily demand per } \\
\text { station } 14\end{array}$ & - & - & - & 12 \\
\hline & Hourly trips (MP) & 838.25 & 743.5 & 939 & 750 \\
\hline & $\begin{array}{l}\text { Hourly demand per } \\
\text { station } 12(\mathrm{MP})\end{array}$ & 7.99 & 6.36 & 7.45 & 5.83 \\
\hline & $\begin{array}{l}\text { Hourly demand per } \\
\text { station } 13(\mathrm{MP})\end{array}$ & - & 2.18 & 3.62 & 3.5 \\
\hline & $\begin{array}{l}\text { Hourly demand per } \\
\text { station } 14(\mathrm{MP})\end{array}$ & - & - & - & 0.48 \\
\hline & Hourly trips (EP) & 1010.25 & 797 & 992 & 751 \\
\hline & $\begin{array}{l}\text { Hourly demand per } \\
\text { station } 12(\mathrm{EP})\end{array}$ & 9.03 & 6.19 & 7.36 & 5.69 \\
\hline & $\begin{array}{l}\text { Hourly trips per station } \\
13 \text { (EP) }\end{array}$ & - & 2.10 & 3.12 & 2.64 \\
\hline & $\begin{array}{l}\text { Hourly trips per station } \\
14(E P)\end{array}$ & - & - & - & 0.33 \\
\hline & Trip length (m) & 1337 & 1268 & 1337 & 1314 \\
\hline & Travel time (mins) & 10 & 10 & 10 & 10 \\
\hline \multirow{16}{*}{$\begin{array}{l}\text { Former } \\
\text { Users } \\
\text { Vs } \\
\text { New Users }\end{array}$} & Daily users & 4193 & 5252 & 3939 & 4311 \\
\hline & Daily trips & 7419 & 9401 & 6786 & 7749 \\
\hline & Daily trips/user & 1.76 & 1.78 & 1.70 & 1.75 \\
\hline & $\begin{array}{l}\text { Daily demand per } \\
\text { station } 12\end{array}$ & 83 & 93 & 68 & 74 \\
\hline & $\begin{array}{l}\text { Daily demand per } \\
\text { station } 13\end{array}$ & - & 46 & 30 & 46 \\
\hline & $\begin{array}{l}\text { Daily demand per } \\
\text { station } 14\end{array}$ & - & - & - & 17 \\
\hline & Hourly trips (MP) & 617.5 & 950 & 653 & 796 \\
\hline & $\begin{array}{l}\text { Hourly demand per } \\
\text { station } 12(\mathrm{MP})\end{array}$ & 5.82 & 7.05 & 5.06 & 5.57 \\
\hline & $\begin{array}{l}\text { Hourly demand per } \\
\text { station } 13(\mathrm{MP})\end{array}$ & - & 3.76 & 2.40 & 3.38 \\
\hline & $\begin{array}{l}\text { Hourly demand per } \\
\text { station } 14 \text { (MP) }\end{array}$ & - & - & - & 1.36 \\
\hline & Hourly trips (EP) & 865.5 & 1085.5 & 775 & 883 \\
\hline & $\begin{array}{l}\text { Hourly demand per } \\
\text { station } 12(E P)\end{array}$ & 7.15 & 7.33 & 5.48 & 6.31 \\
\hline & $\begin{array}{l}\text { Hourly trips per station } \\
13(E P)\end{array}$ & - & 3.93 & 2.19 & 4.12 \\
\hline & $\begin{array}{l}\text { Hourly trips per station } \\
14 \text { (EP) }\end{array}$ & - & - & - & 1.43 \\
\hline & Trip length (m) & 1375 & 1379 & 1337 & 1384 \\
\hline & Travel time (mins) & 11 & 11 & 10 & 10 \\
\hline
\end{tabular}


Table 12 The aggregate use of the system by steady users, former users, and new users on weekends.

\begin{tabular}{|c|c|c|c|c|c|}
\hline \multirow{2}{*}{\multicolumn{2}{|c|}{ Usage metrics (Median) }} & \multicolumn{2}{|c|}{ Mar 12 vs Mar 13} & \multicolumn{2}{|c|}{ Mar 13 vs Mar 14} \\
\hline & & \multirow{2}{*}{$\frac{2012}{4555}$} & \multirow{2}{*}{$\frac{2013}{3449}$} & \multirow{2}{*}{$\frac{2013}{4206}$} & \multirow{2}{*}{$\frac{2014}{3228}$} \\
\hline \multirow{16}{*}{$\begin{array}{l}\text { Steady } \\
\text { users }\end{array}$} & Daily users & & & & \\
\hline & Daily trips & 8127 & 5961 & 7569 & 5304 \\
\hline & Daily trips/user & 1.79 & 1.69 & 1.77 & 1.64 \\
\hline & $\begin{array}{l}\text { Daily demand per } \\
\text { station } 12\end{array}$ & 91 & 61 & 76 & 52 \\
\hline & $\begin{array}{l}\text { Daily demand per } \\
\text { station } 13\end{array}$ & - & 23 & 35 & 29 \\
\hline & $\begin{array}{l}\text { Daily demand per } \\
\text { station } 14\end{array}$ & - & - & - & 9 \\
\hline & Hourly trips (MP) & 895 & 492.5 & 636 & 531.5 \\
\hline & $\begin{array}{l}\text { Hourly demand per } \\
\text { station } 12 \text { (MP) }\end{array}$ & 5.11 & 3.5 & 4.4 & 3.5 \\
\hline & $\begin{array}{l}\text { Hourly demand per } \\
\text { station } 13 \text { (MP) }\end{array}$ & - & 1 & 1.8 & 2.05 \\
\hline & $\begin{array}{l}\text { Hourly demand per } \\
\text { station } 14 \text { (MP) }\end{array}$ & - & - & - & 0.4 \\
\hline & Hourly trips (EP) & 801.5 & 530.5 & 690 & 473 \\
\hline & $\begin{array}{l}\text { Hourly demand per } \\
\text { station } 12(\mathrm{EP})\end{array}$ & 6.56 & 3.75 & 4.6 & 3.2 \\
\hline & $\begin{array}{l}\text { Hourly demand per } \\
\text { station } 13(E P)\end{array}$ & - & 1.55 & 2.25 & 2.0 \\
\hline & $\begin{array}{l}\text { Hourly demand per } \\
\text { station } 14(\mathrm{EP})\end{array}$ & - & - & - & 0.18 \\
\hline & Trip length (m) & 1318 & 1238 & 1295 & 1266 \\
\hline & Travel time (mins) & 11 & 10 & 11 & 10 \\
\hline \multirow{16}{*}{$\begin{array}{l}\text { Former } \\
\text { Users } \\
\text { Vs } \\
\text { New Users }\end{array}$} & Daily users & 4125 & 4823 & 3631 & 3545 \\
\hline & Daily trips & 7189 & 8591 & 6295 & 6073 \\
\hline & Daily trips/user & 1.74 & 1.78 & 1.71 & 1.71 \\
\hline & $\begin{array}{l}\text { Daily demand per } \\
\text { station } 12\end{array}$ & 80 & 85 & 63 & 58 \\
\hline & $\begin{array}{l}\text { Daily demand per } \\
\text { station } 13\end{array}$ & - & 46 & 29 & 36 \\
\hline & $\begin{array}{l}\text { Daily demand per } \\
\text { station } 14\end{array}$ & - & - & - & 15 \\
\hline & Hourly trips (MP) & 484 & 671.5 & 468.5 & 571.5 \\
\hline & $\begin{array}{l}\text { Hourly demand per } \\
\text { station } 12 \text { (MP) }\end{array}$ & 3.89 & 4.4 & 3.1 & 4.2 \\
\hline & $\begin{array}{l}\text { Hourly demand per } \\
\text { station } 13 \text { (MP) }\end{array}$ & - & 2.3 & 1.3 & 2.3 \\
\hline & $\begin{array}{l}\text { Hourly demand per } \\
\text { station } 14 \text { (MP) }\end{array}$ & - & - & - & 1.2 \\
\hline & Hourly trips (EP) & 746 & 830 & 598.5 & 639.5 \\
\hline & $\begin{array}{l}\text { Hourly demand per } \\
\text { station } 12(E P)\end{array}$ & 5.56 & 5.1 & 3.85 & 3.9 \\
\hline & $\begin{array}{l}\text { Hourly trips per station } \\
13 \text { (EP) }\end{array}$ & - & 3 & 1.7 & 2.5 \\
\hline & $\begin{array}{l}\text { Hourly trips per station } \\
14 \text { (EP) }\end{array}$ & - & - & - & 1.07 \\
\hline & Trip length (m) & 1350 & 1337 & 1316 & 1362 \\
\hline & Travel time (mins) & 12 & 11 & 11 & 11 \\
\hline
\end{tabular}




\subsubsection{Spatial distribution of demand before and after system expansion}

\subsubsection{Trends in the spatial distribution of demand and D/S}

In this subsection, we explore the spatial distribution of demand and demand over supply (D/S) before and after the system expansion. As mentioned before, the spatial distribution of hourly demand at stations during morning peak hours is comparable with that during evening peak hours (i.e. high and low demand at stations), we therefore only use daily usage to describe and compare the spatial distribution of demand and $D / S$ between before and after system the system expansion. Figure 16 shows the spatial distribution of demand (i.e. trips/day) by all users on weekdays in March 2012, March 2013, and March 2014. As expected, the demand is the highest in the central part of the city and drops towards the outskirts. Similar trends can also be observed for each user group - former users, steady users, and new users. Figure 17 displays the comparison of the number of daily demands at each station between different user groups on weekdays in March 2012, March 2013, and March 2014. This indicates that stations, which generate a high (and low) demand by one user group, also generate a high demand (and low) demand by another user group. It implies that there is no considerable difference in spatial distribution of demand between different user groups. Moreover, Figure 18 shows the spatial distribution of statistically hot spots of demand (i.e. trips/day) by each user group on weekdays in March 2012, March 2013, and March 2014. This further confirms that there are no substantial differences in the pattern of spatial distribution of demand between different user groups. The statistically significant hot spots are generally the same among different user groups (i.e. following the system expansion). This suggests that the spatial distribution of demand before the system expansion is comparable with that after the system expansion, as well as between different user groups. In all cases, high-demand areas concentrate in the centre, whereas the lowdemand areas are on the outskirts. This might be attributed to the fact that the central area has the highest density of population, bike stations, and mixed land use patterns. 


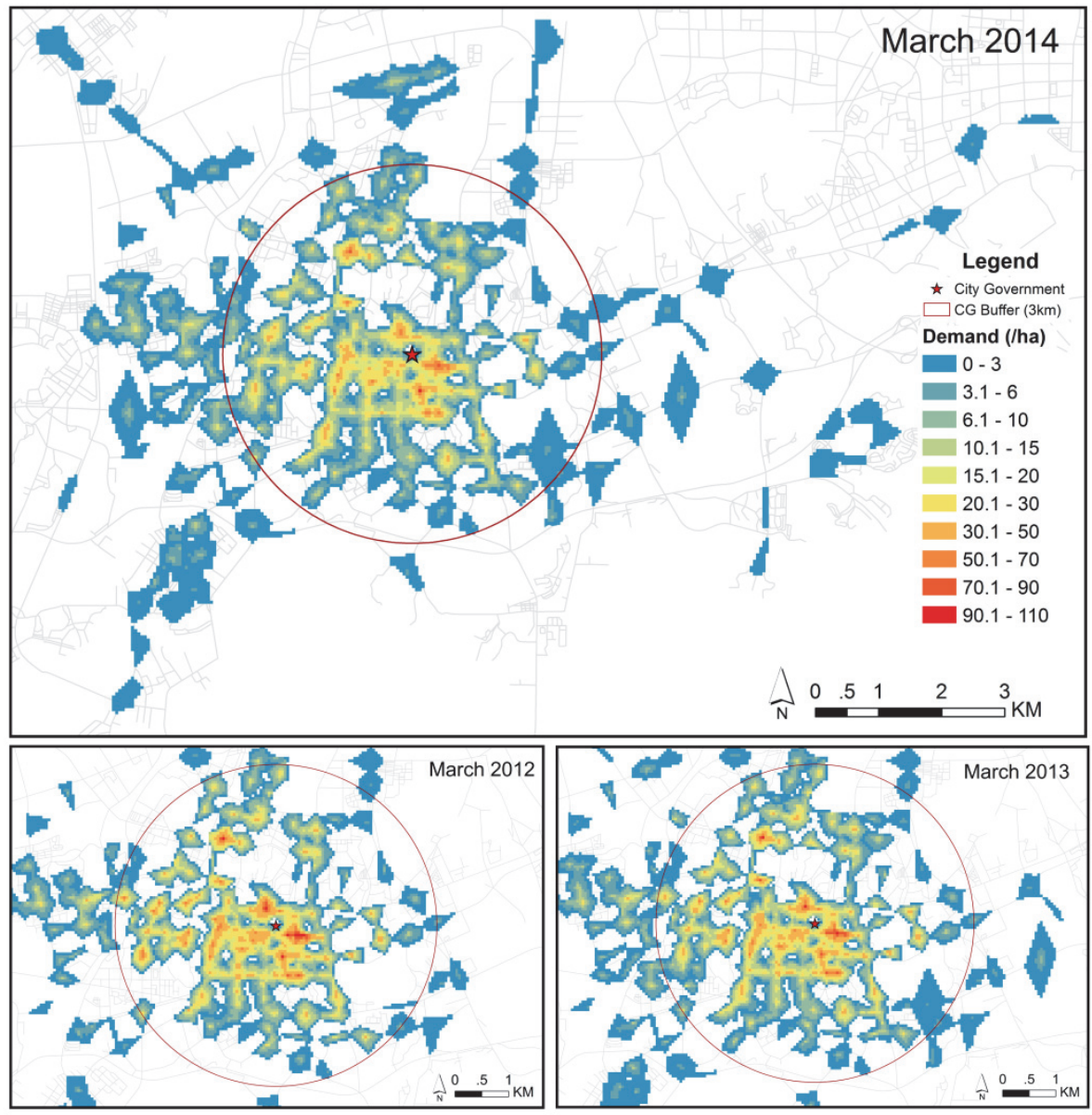

(1)The upper panel represents the result of March 2014. (2)The lower panel represents the results of March 2012 (left) and March 2013 (right).

Figure 16 The spatial distribution of demand by all users on weekdays. Demand represents the number of trips per day. 

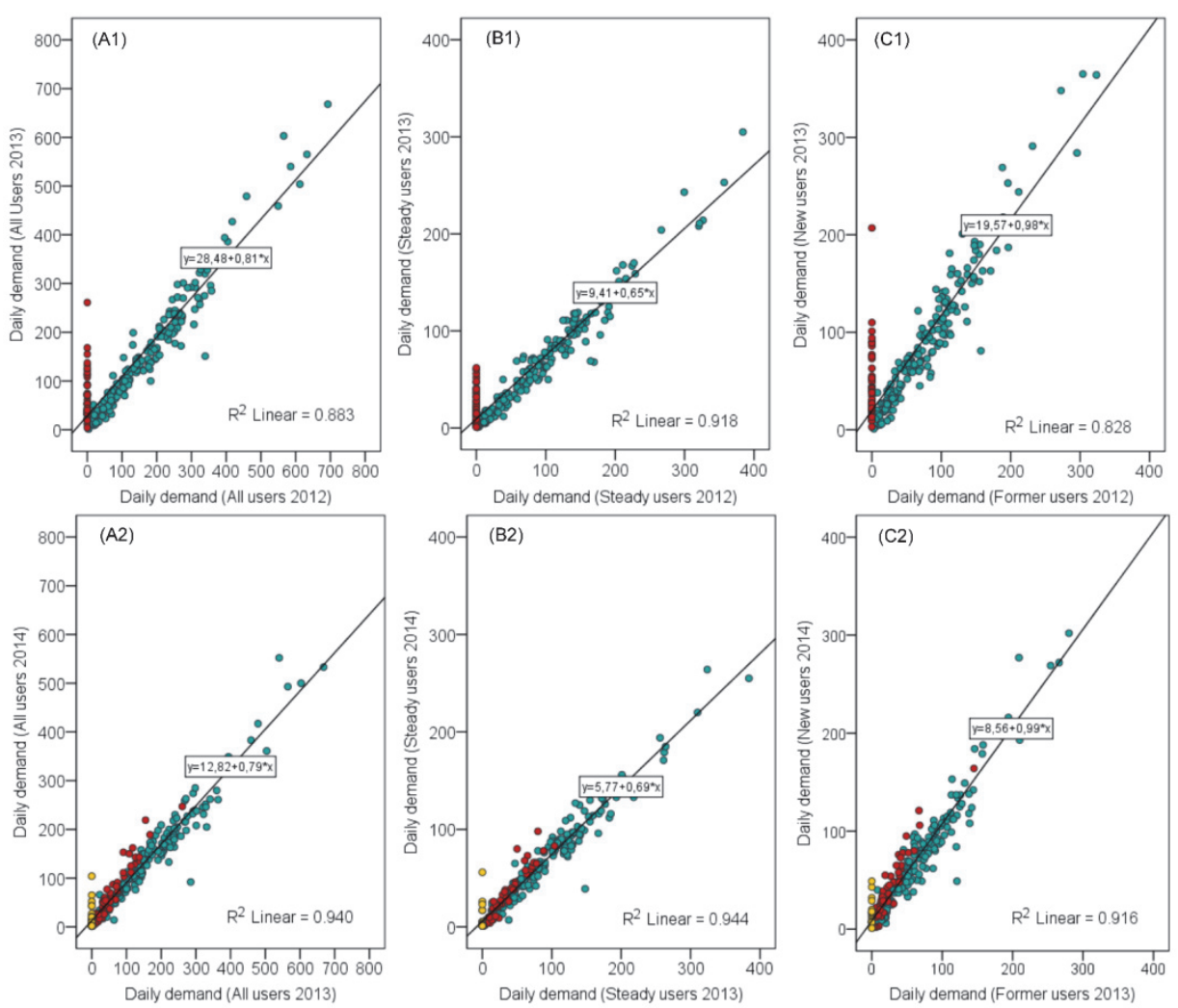

(1) The points represent each of bike stations. (2) Blue, red, and yellow symbols denote "stations 12", "stations 13", and "stations 14" respectively. (3) Figures A describes the user groups of Mar 2012 versus Mar 2013, and figures B describes user groups of Mar 2013 versus Mar 2014.

Figure 17 Comparisons of daily demand by one group and another group at stations (weekdays). 


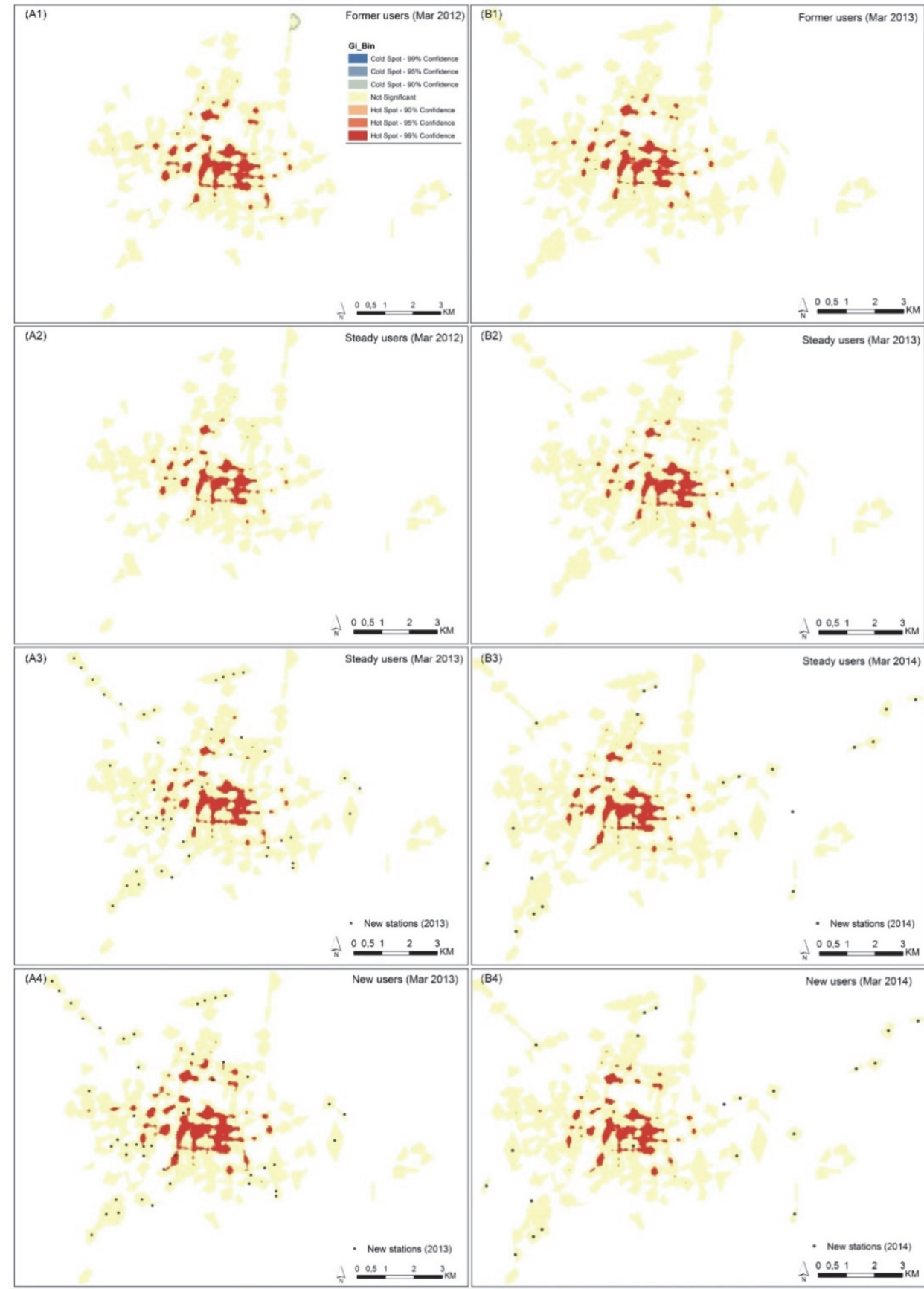

(A) Describes the user groups of Mar 2012 versus Mar 2013, and (B) describes user groups of Mar 2013 versus Mar 2014.

Figure 18 Getis-Ord Gi* statistic of the spatial distribution of demand by each user group (weekdays). 
Figure 19 shows the spatial distribution of D/S (i.e. the ratio of trips/day to slots) on weekdays in March 2012, March 2013, and March 2014. Not surprisingly, it somewhat follows the trend in demand with high $D / S$ in the central area and low D/S on the outskirts. The figure however shows an overall decrease in D/S following the system expansion, especially in the central area, the colour changes from upper class to lower class (such as from 7.1-12 to 6.1-7). This might be attributed to the overall decrease in demand by all users following the system expansion. In addition, some of areas, which showed a high D/S before system expansion, decreased after building a new station nearby, such as marked in the areas A, B, and C shown in Figure 8 . The overall demand in those areas has increased after system expansion, due to the demand for new stations. This suggests that new stations might compete with nearby older stations, resulting in mitigating the excess demand (finding available bikes or empty slots) in areas that had a high D/S before the system expansion.
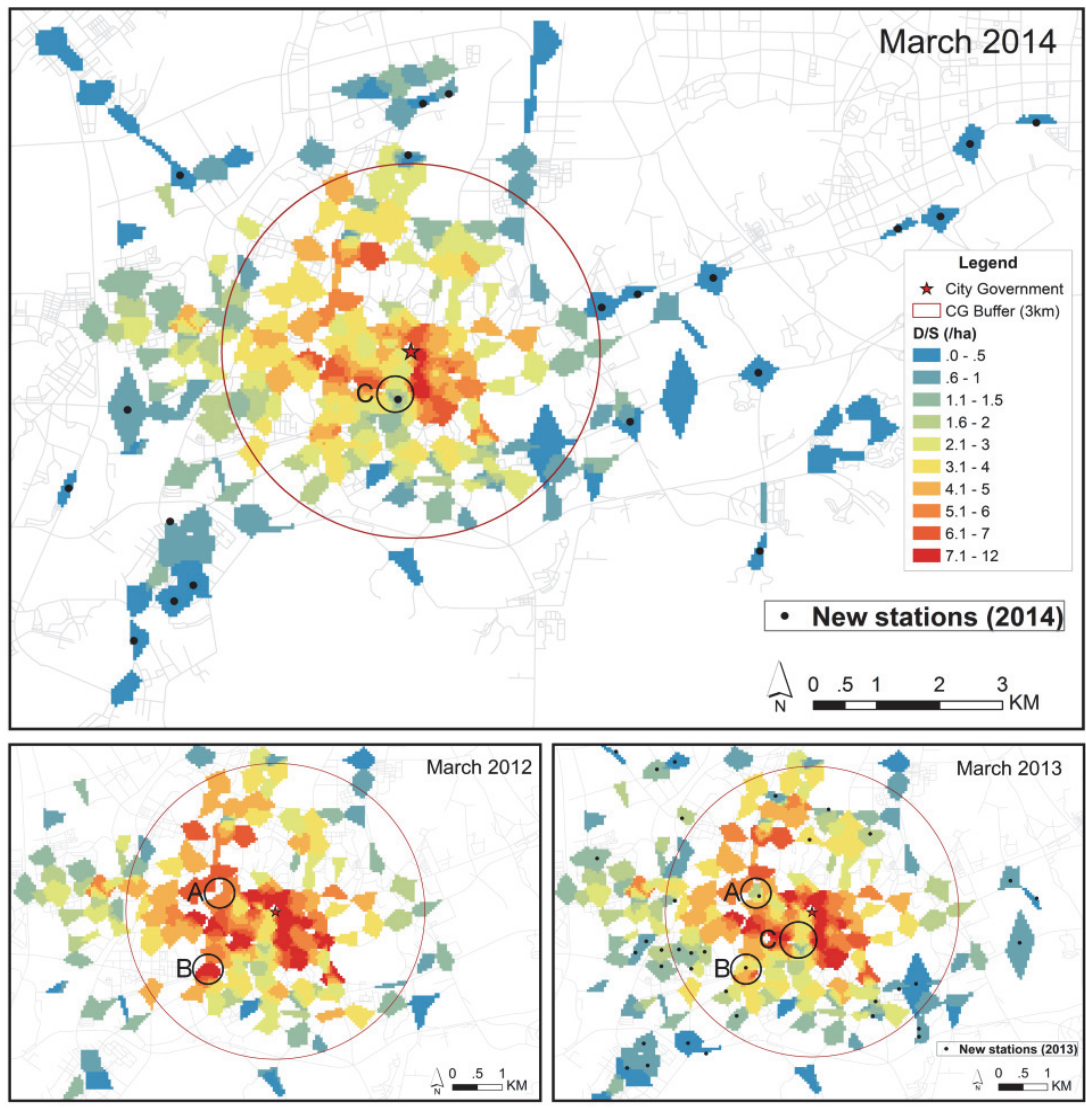

(1)The upper panel represents the result of March 2014. (2)The lower panel represents the results of March 2012 (left) and March 2013 (right)

Figure 19 The spatial distribution of $D / S$ by all users on weekdays. The $D / S$ represents the ratio of trips/day to the number of parking slots. 
In the previous subsection, we found that the overall use of the system has decreased more on the weekends than on weekdays. Therefore, we examined the spatial difference in demand between weekdays and weekends. The results are shown in Figure 20. Although the overall spatial distribution looks quite similar for weekends and weekdays, Figure 20 shows there are differences in the number of demands. The red areas show a relatively higher demand on weekdays, while the blue areas show relatively higher demand on weekends. The figure shows that these areas are more or less the same in the three years.
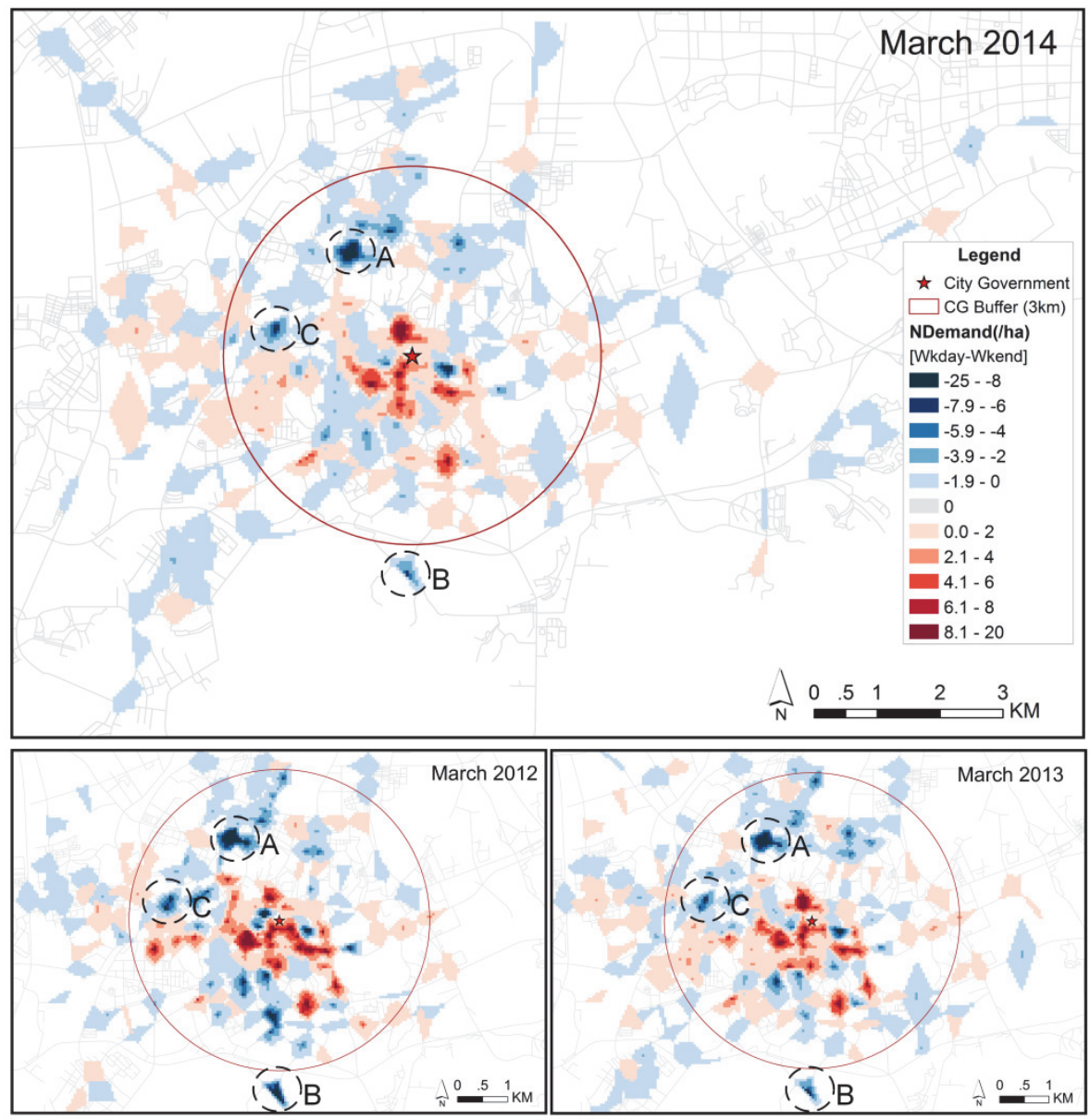

(1)The upper panel represents the result of March 2014. (2)The lower panel represents the results of March 2012 (left) and March 2013 (right).

Figure 20 Differences in spatial demand by all users between weekdays and weekends. The "NDemand" represents the difference in normalized demand between weekdays and weekends.

This result suggests that differences between weekdays and weekends are not related to the expansion of system, but probably to the surrounding built 
environment. Blue areas are mainly occupied by shopping malls or parks, distributed far from the city centre, marked as areas A, B, and C in Figure 20. The significant red areas are mainly located in the city centre, with relatively many offices and residential communities. This implies that commuting is more dominant on weekdays, and shopping and recreation are more important purposes in the weekends. These results suggest that the demand in an area is influenced by the nearby dominant land use type.

\subsubsection{Differences in spatial demand by user-groups}

In this section, we focus on two aspects. The differences in spatial demand between the three years are shown in Figure 21 . This is done for all users (upper panel), new versus former users (centre panel), and for steady users (lower panel). The differences in spatial demand between new users and steady users after the system expansion are shown in Figure 22.

The red areas in Figure 21 show that the demand after system expansion is higher than before the system expansion (higher in 2013 than in 2012, left panel; and higher in 2014 than 2013, right panel). The blue areas are areas in which demand has decreased. Note that the most significant increases (illustrated by deep red colour) are in areas with newly-built stations. According to Figure 21, decreases in demand are mainly observed in central areas. We highlight areas with the most significant decrease (i.e. more than 4 times the standard deviations below the average normalized difference of 0 ) in each figure. These areas are not necessarily the same when comparing the second expansion with the first one, or when comparing new users (vs. former users) with steady users.

Area $A$ is a specific case. The strong decrease in 2013 (compared to 2012) is due to the removal of a station. The other stations show real decreases in demand. Area B, C, and E show a significant decrease throughout all years, and area B for all groups but area $C$ and $E$ for steady users. However, these areas are constantly high-demand areas throughout three years, area $\mathrm{B}$ and $\mathrm{E}$ are occupied by a shopping mall and area $C$ is occupied by a mix of offices and residential communities. The continuous decline of demand in these areas might be attributed to the negative performance of the system, such as the quality of bikes is not as good as the beginning, and unavailability of bikes or parking slots.

Additionally, decreases in other areas area only significant in one of the two expansions. However, we observe a decrease in all cases. It should be noted that demand - by both new users (vs. former users) and steady users - has decreased in areas where (many) new stations were added nearby. This is in particular the case for area D, F and J that are occupied by the mix of offices and residential communities. The case for area $G$ that is a commercial area 
consisting of hotels, shopping malls, and entertainment venues, where a new station was added nearby in the first expansion, decreased demand in 2013 (left panel) and shows a significant decrease of demand after the second expansion (right panel). However, the demand in newly-built areas increased after second expansion (right panel). This implies that there might be competition between nearby stations, where newly-built stations are more attractive than the older stations. Two specific areas $\mathrm{H}$ and $\mathrm{I}$ - that are occupied by residential communities (area $\mathrm{H}$ ) and the mix of colleges, residential communities and a park (area I) - only show a significant decrease in demand by new users (vs. former users) after system expansion. This might be due to fewer new users have demand for stations in these areas, such as people living, studying or working in this location.

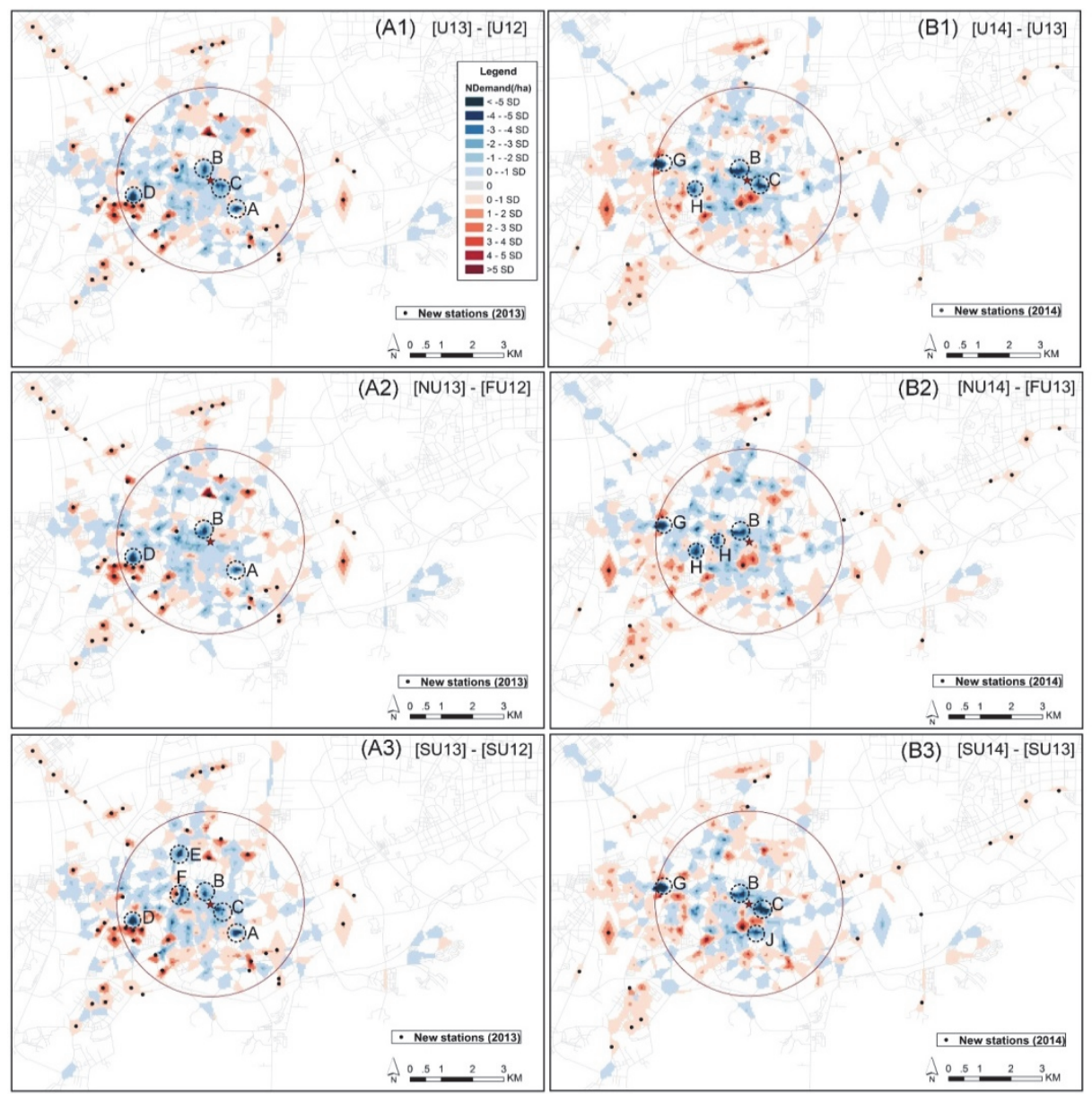

(1)The "NDemand" represents the difference in normalized demand between user groups. (2)The left panel represents the comparison between March 2012 and March 2013, and right panel represents the comparison between March 2013 and March 2014.

Figure 21 Differences in spatial demand between user groups of before and after the system expansion (weekdays). 
Figure 22 shows the differences in spatial demand between new users and steady users after system expansion, i.e. comparing new users with steady users in March 2013 (left panel), and in March 2014 (right panel). March 2013 and March 2014 show similar patterns. In the blue areas the demand is relatively high for steady users, while in the red areas the demand is relatively high for new users. For the majority of areas, the difference in demand between steady users and new users is not very high. Figure 22 illustrates that steady users show the higher demand for both old stations and new stations that are located in the city centre, such as the newly-built areas $\mathrm{M}$ and $\mathrm{N}$. This can be attributed to the fact that the activities of steady users were mainly concentrated in the central area before the system expansion; users have more desires for newly-built stations in this area rather than the new stations that are far away. New users generated a higher demand at the majority of new stations as well as at some old stations nearby shopping malls - as areas $\mathrm{E}$ and $\mathrm{K}$. This implies that adding new stations in the areas where demand or density of stations is high, both new users and original users can be attracted. On the other hand, adding new stations in areas further away from the city centre, with a lower density of stations, is mainly useful for new users rather than steady users. In general, expanding the original system not only extends the original users' ability to reach new areas but also attracts new users to use bike-sharing systems.

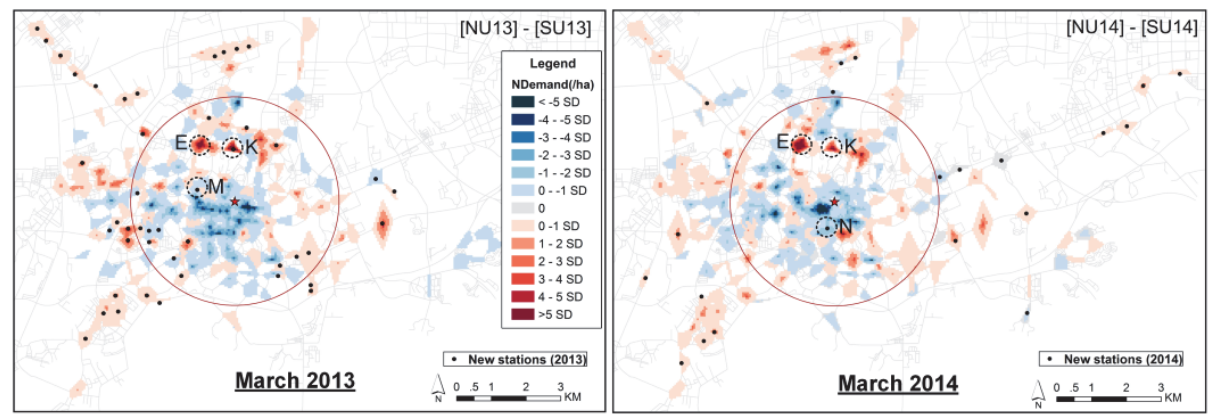

(1)The "NDemand" represents the difference in normalized demand between new users and steady users. (2)The left panel represents the comparison between new users and steady users in March 2013. The right panel represents the comparison between new users and steady users in March 2014.

Figure 22 Differences in spatial demand between new users and steady users after system expansion (weekdays).

\subsection{Conclusions}

This study has investigated how the system usage has changed over the years and how the system expansion affects the usage of the system. It was performed to evaluate Zhongshan's bicycle-sharing system, using trip data from March 2012, March 2013, and March 2014. The system gradually expanded the number of stations equipped with parking slots from March 2012 
to March 2013 and then again from March 2013 to March 2014. We conducted both a statistical and a spatial analysis to examine the changes in both users and system usage between before and after the system expansion, namely March 2012 versus March 2013 as well as March 2013 versus March 2014. The system usage was measured by: (1) the aggregate use of the system; and (2) the spatial distribution of users' demands and the ratio of demand to supply (D/S). In addition, travel on weekdays was analysed separately from travel on weekends.

There has been a great variation in the number of users over the years, with only $45 \%-46 \%$ of all users - steady users - continuing to use the system after the system expansion. Many users - former users - stopped using the system, and many new users started to use the system after the system expansion. Moreover, there are overall decreases in the system usage by all users after the system expansion compared to before the system expansion, due to the overall decreases in the system usage by steady users after the system expansion, although new users used the system more frequently than former users.

There is no significant difference of the trend in spatial distribution of both demand and D/S between, before and after the system expansion. The highdemand areas concentrate in the centre and are occupied by old stations, and the low-demand areas are on the outskirts. This is attributed to the fact that the centre area has the highest density of population, bike stations, and mixed land use patterns. However, there were decreases in demand in most highdemand areas over the years, due to a reduced demand by both steady users and new users (versus former users). This implies that stations in these highdemand areas did not work well after the system expansion compared to before the system expansion, which is not attributed to the system expansion, but might be caused by the fact that the novelty was gone for some steady users or the negative performance of the system, such as the quality of bikes not being as good as in the beginning, and unavailability of bikes or parking slots.

In some areas which are occupied by both old and new stations after the system expansion, less demand by both new users and steady users was generated at these old stations after the system expansion, compared with the demand by former users and steady users before the system expansion. Moreover, the spatial distribution of $D / S$ reveals that these areas showed a high D/S before the system expansion, but decreased the D/S after building a new station. This suggests that nearby stations might be competing with each other, and building new stations in former high D/S areas can contribute to easing the excess demand in these areas. In addition, the difference in demand over the urban area between weekdays and weekends reveals that users might 
cycle mainly for commuting on weekdays, but for shopping and recreation on weekends.

In general, expanding the original system not only extended the original users' ability to reach new areas but also attracted new users to use the bike-sharing system. Adding new stations in the areas where demand or density of stations is high can attract both new users and original users. On the other hand, adding new stations in areas further away from the city centre with a lower density of stations is mainly useful for new users rather than steady users.

With the development of a bike-sharing system, to improve the system and make it more sustainable rather than a short-lived project, this study is aligned with a tendency for operators and researchers to investigate the system usage and travel behaviours of bike-sharing users by the trip data that discloses more information than the station-based data. That was also the motivation for us to conduct this study. To be sure, this study is not without limitation. Due to the data limitation, we only compared the one-moth system usage between three years. It would be better to collect and analyse the trip data over the long term, which may make the results of analysis more conclusive. This is an avenue for future work.

For further expansion of bike-sharing systems, we suggest that it would be better to first investigate the spatial patterns of users' demands and system's supply to uncover the high and low level of demand as well as the ratio of demand to supply across the urban area. Next, we suggest building new stations in the area that has an excessive ratio of demand to supply rather than expand the system to new areas unless there is a clear necessity for serving new areas. Building new stations in the areas with high ratio of demand to supply not only extends the service area of the system but also mitigates the difficulty of finding a public bike or a parking slot. 


\section{Chapter 5}

\section{The travel behaviour of bike-sharing users ${ }^{4}$}

4 This chapter is based on Zhang, Y., Brussel, M.J.G., Thomas, T., van Maarseveen, M.F.A.M. (2017). Mining bike-sharing travel behaviour data: an investigation into trip chains and transition activities. Computers, Environment and Urban Systems, (under review). 


\begin{abstract}
Bike-sharing is booming. The latest generation of bike-sharing programs offers an access to trip-based usage data, which enables researchers to explore the travel behaviour of cyclists. Previous trip-based researches mainly focus on the analysis of origins and destinations of bike-sharing trips. However, most such studies neither consider the transition activities between consecutive trips within a trip chain, nor incorporate the information of land use types and time relating to the start and end of individual trips. To address these issues, we conducted this study to elucidate the travel behaviour of bike-sharing users, using 5 months of trip data from a bike-sharing system in Zhongshan (China). To this end, we constructed origin-destination matrices and transition matrices, incorporating land use types of bike stations and time information of pickup and drop-off activities. We found that the majority of trips are directional trips that start and end at different stations. The dominant pattern of trip chains is the loop trip chain that might relate to commuting. Moreover, the majority of transition activities show that users depart from the same station where they arrived during their previous trips. In addition, findings from the matrices of individual trips, trip chain and transition activities suggest that bike-sharing trips are primarily related to work, home, and shopping. In the conclusions, we present and discuss the findings in detail. We believe that other cities with bike-sharing systems can draw inspiration from this study's methods and findings.
\end{abstract}

Keywords: bike-sharing trips, trip chains, transition activity, travel behaviour, travel purposes 


\subsection{Introduction}

As a pinnacle of green and public transport, bike-sharing is booming. One of the main advantages of bike-sharing programs is that people use public bicycles on an "as-needed" basis without the costs and responsibilities (e.g. storage, repair, etc.) of bicycle ownership (Ricci, 2015; Shaheen et al., 2010). Moreover, researchers also found that many of bike-sharing users own a private bicycle, and there is a complementary relationship between private bicycle and public bicycle in regard to travel distance (Castillo-Manzano et al., 2015; O'Neil and Caulfield, 2012). Evidence has shown that cycling for both utilitarian and recreational purposes has increased in some cities after running bike-sharing programs (Fishman et al., 2013; O'Brien et al., 2014). Until 2016, a total of 1090 bike-sharing systems were running in 60 countries worldwide, many more will take off in the near future (Meddin and DeMaio, 2016).

Bike-sharing usage can vary dramatically between different systems (Fishman, 2016). The role of bike-sharing systems is often understood as a supplement to other forms of public transport (DeMaio, 2009; Shaheen et al., 2012). However, as based on a review of international programs, this is not always the case. Evidence from numerous systems has shown that much of the bikesharing usage has been a substitute for walking and other public transport rather than for private vehicle use, (Bachand-Marleau et al., 2012; Bullock et al., 2017; Fishman, Washington, \& Haworth, 2014; Martin and Shaheen, 2014; Shaheen et al., 2011). Despite this, some systems, which mainly serve the city centre (e.g. Dublin) or the suburban areas (e.g. the system in Jiangning district of Nanjing, China), show the synergy of bike-sharing and other public transport due to users need to commute between the central and suburban areas(Jiménez et al., 2016; Zhao et al., 2015). The condition of other public transport has a significant impact on the use of bike-sharing (Yang and Long, 2016). As stated by Kager et al. (2016), cycling and public transport have a symbiotic relationship, which cannot be treated separately.

Bike-sharing usage data enable researchers and planners to explore travel behaviour on a continuous, large scale, and non-invasive way (Beecham and Wood, 2014). To gain insights into the characteristics and patterns of bikesharing trips, previous studies either focused on trip characteristics in terms of travel speed and time (Jäppinen et al., 2013; Jensen et al., 2010) and the usage types of bike-sharing trips (Bordagaray et al., 2016), or focused on employing visualization techniques to explore the gendered travel behaviour (Beecham and Wood, 2014; Zhao et al., 2015), spatial structure of bikesharing trips (Zaltz Austwick et al., 2013) and commuting dynamics (Oliveira et al., 2016). Those studies can generally be summarized as movement-based research, without considering the transition activities between consecutive trips within a trip chain. Mining the travel behaviour and patterns of bikesharing usage solely depending on the individual trips (i.e. origin and 
destination) could be biased, as the sequence of activities also provides information on the mobility patterns (Wu et al., 2014). Moreover, previous studies mainly emphasized the strong origin-destination pairs and patterns, without incorporating the geographical and time information relating to the start and end of the trips. However, the location and time relating to the origin and destination of individual trips are essential for understanding the travel behaviour (purpose) of bike-sharing usage.

Within this context, to elucidate the travel behaviour of bike-sharing usage, this paper examined the spatial and temporal characteristics of bike-sharing trips, trip chains, and transition activities. The spatial characteristic was investigated by the land use type of each bike station, and the temporal characteristic was investigated by the time information relating to the start and end of individual trips, trip chains, and transition activities. In this study, the term "transition activities" means the drop-off activity and pickup activity between consecutive trips within a trip chain. This study was conducted for a bike-sharing system in Zhongshan (China), using 5-months of trip data from February 2014 to June 2014.

The remainder of this paper is organized as follows: section 5.2 presents the previous studies on travel behaviour of bike-sharing usage, section 5.3 introduces the transport condition and bike-sharing system in study area, section 5.4 explains the data of bike-sharing usage and methods of this study, section 5.5 presents and discusses the results, and section 5.6 concludes the paper.

\subsection{State of the art researches in travel characteristics of bike-sharing usage}

Numerous and various studies have investigated the travel characteristics of bike-sharing users, which shed light on the usage and the role of such systems within different urban backgrounds. Previous researches can be generally classified into three categories: (1) user survey-based studies; (2) stationbased studies; and (3) trip-based studies.

\section{- User survey-based studies}

User survey-based studies were done through interviews and questionnaire surveys on a sample of bike-sharing users, to understand users' profile, perceptions and travel behaviour. Referring to travel behaviour, several findings have been observed. Firstly, many studies have found that bikesharing trips are mainly substitutes for walking and public buses, rather than for private vehicle use (Bullock et al., 2017; Murphy and Usher, 2015; O'Neil and Caulfield, 2012; Shaheen et al., 2011; Tang et al., 2011). The primary 
mode (walking or public transport) that is replaced by a bike-sharing system is different in different cities. For example, $85 \%$ of Dublin's bike-sharing users (respondents) substitute bike-sharing for walking (54\%) and public transport (31\%) (O'Neil and Caulfield, 2012). Tang et al. (2011) indicated that $22.73 \%$ and $34.42 \%$ of Beijing's bike-sharing trips shift from walking and public buses, and $26.15 \%$ and $40.37 \%$ of Shanghai's bike-sharing trips shift from walking and public buses respectively. About $80 \%$ of Hangzhou's bike-sharing users (respondents) shifted from public transport to bicycle use (Shaheen et al., 2011). Secondly, although users used public bikes for both transport and recreational activities, the most common bike-sharing travel purpose is workrelated and school-related (Shaheen et al., 2012; L. Zhang et al., 2015). Thirdly, bike-sharing systems are not consistent in acting as a feeder mode to existing public transport. The role of bike-sharing systems can vary between different cities. Referring to American and European cities, Martin and Shaheen (2014) stated that Washington DC's bike-sharing system led to a fall in public transport use (bus and rail) in the dense central urban area, but increased the public transport use in suburban areas, due to the fact that trips are shorter and that there are more stations in the central urban area and that bike-sharing trips can aid rail ridership in the suburban areas where trips are longer. Fishman, Washington, Haworth, et al. (2014) found that Melbourne's system was potentially substituting for public transit rather than connecting to it. Nikitas et al. (2016) indicated that Gothenburg bike-sharing system is a good travel alternative to a car for inner-city trips. Jiménez et al. (2016) suggested that Dublin's bike-sharing system, which is mainly restricted to central urban areas, can be used as a complement to other public transport due to many commuters arriving in city core by other public transport (bus, tram, train). Referring to the Chinese bike-sharing systems, Shaheen et al. (2011) indicated that Hangzhou's bike-sharing system, which covers the whole urban area, acted as both a competitor and a complement to the existing public transit. For the bike-sharing system in Jiangning district, which is a suburb area of Nanjing, Zhao et al. (2015) indicated that many trips were connecting with rail, and public bikes also serve as alternatives for moderate-distance trips in such areas. In the city of Zhuzhou (a medium-sized city of China), L. Zhang et al. (2015) indicated that a significant proportion of users choose public bikes to complete their entire urban trip. An important fact is that most Chinese systems provide a one-hour free of charge time, which is enough for most single trips (Tang et al., 2011).

\section{- Station-based analyses}

Station-based analyses mainly aim at exploring the usage pattern of bike stations. Some studies have examined the patterns of usage activities at bike stations and have classified stations into several clusters (Jiménez et al., 2016; Kaltenbrunner et al., 2010; O'Neil and Caulfield, 2012; Vogel et al., 2011). For 
example, Vienna's bike-sharing stations are grouped into five clusters according to the pickup and return at each station in the daily course of working days (Vogel et al., 2011), and Dublin's bike stations are grouped into three clusters in terms of generator stations, attractor stations and balanced stations (Jiménez et al., 2016). Some studies have examined the effect of surrounding built environment on the demand at bike stations. They generally found that population and job density, the proximity to metro and public bus stations, bike lanes and points of interests (retail shops, restaurants, parks, etc.) are positively associated with the demand at stations (El-Assi et al., 2015; FaghihImani et al., 2014; Gonzalez et al., 2016; Y. Zhang et al., 2017). Moreover, station size and nearby other bike stations within the catchment area also have effects on the demand at stations (El-Assi et al., 2015; Faghih-Imani and Eluru, 2015; Y. Zhang et al., 2017). Additionally, slope is also a key barrier for station usage, i.e. stations located at higher elevations leading to rebalancing problem due to few bikes are returned to hilltops (Mateo-Babiano et al., 2016).

\section{- Trip-based analyses}

Trip-based analyses give a better insight into the characteristics of individual trips, such as travel speed and duration and trip-based movement which offers much more room for investigating the travel behaviour of bike-sharing users. Some studies analysed the travel speed, time and distance of cycling trips. Jensen et al. (2010) found that public bikes compete with the car in terms of speed in downtown Lyon, and Jäppinen et al. (2013) found that the adoption of a bike-sharing system can reduce about $10 \%$ of travel time by public transport in Greater Helsinki, on average by more than $10 \%$. Moreover, MateoBabiano et al. (2016) uncovered that distinctive Brisbane's bike-sharing trips show a clear morning and evening peak hours on weekdays and shorter trips on weekends than weekdays.

Apart from this, other trip-based studies explored the movement of bikesharing trips. Studies on the movement of biking trips mainly focused on the visualization techniques. The common idea of these movement analyses is to weight the trips between two stations to emphasize the strong OriginDestination pairs and patterns. The visualization of trajectories shows the bicycle flow over the urban area, which is often represented by lines with a different thickness. Zaltz Austwick et al. (2013) employed visualization techniques to explore the travel behaviour of bike-sharing usage in five different cities. They found that systems show similarity in the distribution of trip displacements and durations. However, they did not incorporate information of trip time and any land-use data of stations. Beecham and Wood (2014) proposed an approach to visualizing the flow of London's bike-sharing trips, and found that women tend to use public bikes at weekends and in areas with cycle routes and/or slower traffic, while men tend to use public bikes for 
commuting. Similar visual techniques were employed by Zhao et al. (2015) who analysed Nanjing's bike-sharing trip chain that was divided into two major types - O-O (O-smD-O) and O-D (O-smD), and uncovered that women tend to make multiple-circle trips and spend more time on cycling than men on weekdays. However, they neglect one potential problem: the distance between the Origin station $(O)$ of the first trip and final destination station of the last trip (D). It is quite possible that users want to drop off the bikes at the original station, but that such a station is full (no parking slots) so that users have to choose a nearby station to return the bikes. In that case, the O-D type could be the potential O-O type. Bordagaray et al. (2016) classified bike-sharing trips into five usage types in terms of round trips (same origin and destination), bike substitution, and perfectly and non-perfectly symmetrical trips, using trip data of Santander's bike-sharing system. The found that $53.9 \%$ of trips made by registered users and $47 \%$ trips made by casual users belong to non-classified usage types, while two predominant usage types are round trips and perfectly symmetrical trips. Oliveira et al. (2016) designed an interactive visualization system to explore the commuting dynamics of New York's bike-sharing system. They mainly aimed for exploring the station balance over time, i.e. identifying whether the condition of station capacity is full or empty. However, they did not consider the geographical information (e.g. points of interest, land use types) relating to the start and end of the trip, which can be beneficial to identifying the potential trip purpose.

To summarize, the existing trip-based researches mainly focus on the characteristics of cycling movements, but neglect transition activities within the trip chain that might be the driving force underlying cycling movements. Moreover, few studies considered the land use type and time information relating to the start and end of individual trips and trip chains. A major limitation of the operational usage data is that they do not provide the reason for trip, but bike-sharing trips vary based on land uses (Ahillen et al., 2016; Lathia et al., 2012; Mateo-Babiano et al., 2016). One cannot understand and predict the travel purpose only according to the movement, i.e. OriginDestination bike flows. Within this context, to elucidate he travel behaviour of bike-sharing usage, this study explores the patterns of individual trips, trip chains, and transition activities, incorporating the land use type and time information relating to the start and end of individual trips, trip chains, and transition activities. The synthesis of the three aspects is an indication of potential travel purpose of bike-sharing usage. 


\subsection{Study area}

\subsubsection{Transport condition in Zhongshan, China}

Zhongshan city is a prefecture-level city located in the Guangdong province of China. As shown in Figure $23(\mathrm{~A})$, the city government directly administers six districts corresponding to the urban area, and eighteen towns. Moreover, four districts, the Xi, Shiqi, Dong, and Nan districts, constitute the "centre urban area" $(172.7 \mathrm{~km} 2)$, in which the Xi, Shiqi and Dong district are the political and cultural centre of the city and are mainly occupied by the service industry and the Nan district holds the manufacturing and service industries. One of the other two districts, the Torch Hi-tech Industrial Development district $(90 \mathrm{~km} 2)$, is a national-level hi-tech industrial development zone, and the Wuguishan district $(113 \mathrm{~km} 2)$ is mainly for tourism and agriculture. As shown in Figure 23 (B), the largest population is in the core of "centre urban area", and the smallest population in the Hi-tech and Wuguishan district. As shown in Figure 23 (C), which describes the distribution of Housing-Job ratio across TAZs, which was derived from the Zhongshan Household Travel survey in 2010, the housing and job are quite comparable in most TAZs.

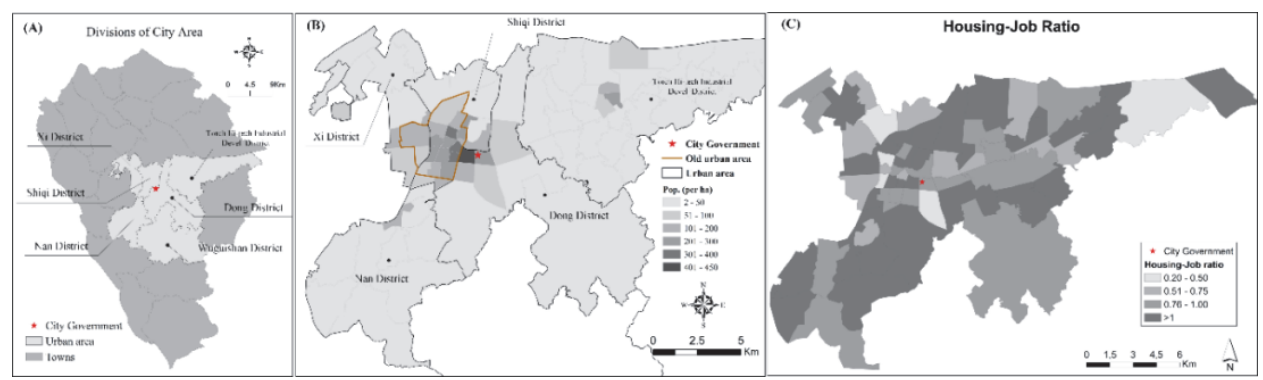

(A) Division of city area. (B) Population density distributed in the urban area. (C) Housing-Job ration in TAZs.

Figure 23 City Background.

According to Zhongshan Household Travel survey in 2010 that was done at the level of Transport Analysis Zone (TAZ), it indicates that no matter which type of commuting trips, the average duration of commuting trips is quite short (i.e. less than 20 minutes) in the urban area. Figure 24 shows the modal split (four primary modes) in each TAZ. The share of different modes indicates that: nonmotorized $>$ motorcycle $>$ car $>$ public bus. The share of non-motorized mode is much larger than that of public bus. This suggests that commuting by nonmotorized transport was the common lifestyle even before running the bikesharing system, and the public bus is not attractive to citizens neither in the inner area nor on the outer area. In the light of commuting time and modal split, it suggests that local people prefer to commute by walking and biking, and tend to live close to workplaces in general. 

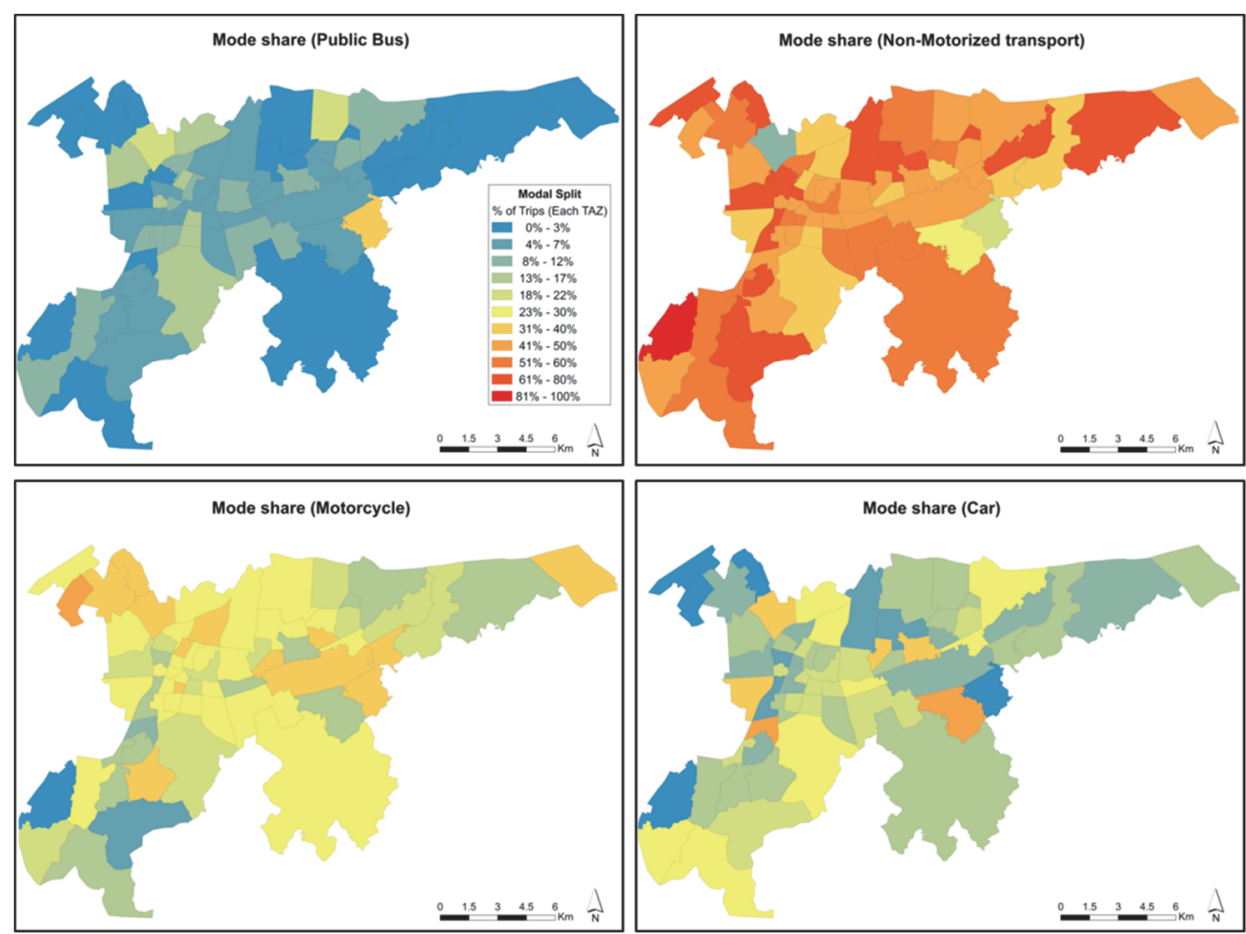

Figure 24 Modal split of commuting trips in TAZs.

\subsubsection{Zhongshan's bike-sharing system}

Zhongshan's bike-sharing system was launched in 2011 and is a 24/7 selfservice system. Users can pick up and return public bikes at any station in the course of the day by use of a smart card after membership registration. For each trip, the first hour is free, and the rest of the hours are charged at incremental prices (1CNY per hour), which is quite a lot cheaper than a trip by local public bus ( 2 CNY per trip). The system gradually expanded over the urban area, i.e. from the central urban area to the outer urban areas. Until June 2014, 296 bike stations, equipped with 7855 parking slots were distributed over the urban area (as shown in Figure 25); 224 of these stations had been built before 2014 and are mainly located in the "centre urban area", and 72 bike stations were built in 2014 and are mainly located in "Torch Hitech Industrial Development district". 


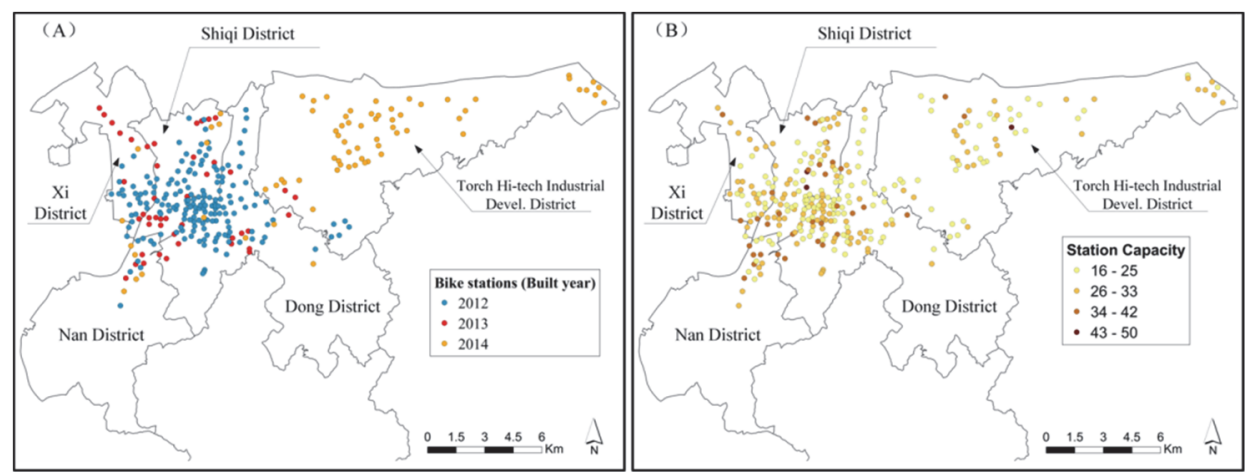

Figure 25 (A)The spatial distribution of bike stations and (B) station capacity .

\subsection{Data and Methods}

\subsubsection{Bike-sharing trip data}

The trip data were collected from the Transport Department of the Urban Planning and Design Institute of Zhongshan (China). The provided trip database consists of usage information from February to June 2014 (5 months). Each piece of usage information (i.e. each trip) includes user ID, pickup and return stations, and start time and end time of the trip. Using the latter, the duration of each trip is calculated by subtracting the start time from the end time. Moreover, each user ID is unique and connected with the smart card. Referring to the original trip database that records the usage of public bikes from February to June 2014, there are 1,937,265 records (i.e. trips), generated over the urban area in these 5 months. Based on data screening, we excluded $6 \%$ of inaccurate records from the original trip database, which included $5.88 \%$ of trips that had a pickup and return at the same station with a duration of less than 1 minute, and $0.12 \%$ of trips that had a duration of less than 1 minute. Figure 26 shows the number of trips arrival at and departure from each station during morning peak, evening peak, and off peak hours of weekdays. According to Figure 26, pickup activities and drop-off activities are quite comparable for both regional and hourly (peak and off-peak hours). Central stations show high demands, and outer stations show low demands, for both pickup and drop-off activities. This is quite different from other cities that show an imbalanced trip flow, both regional and hourly. 


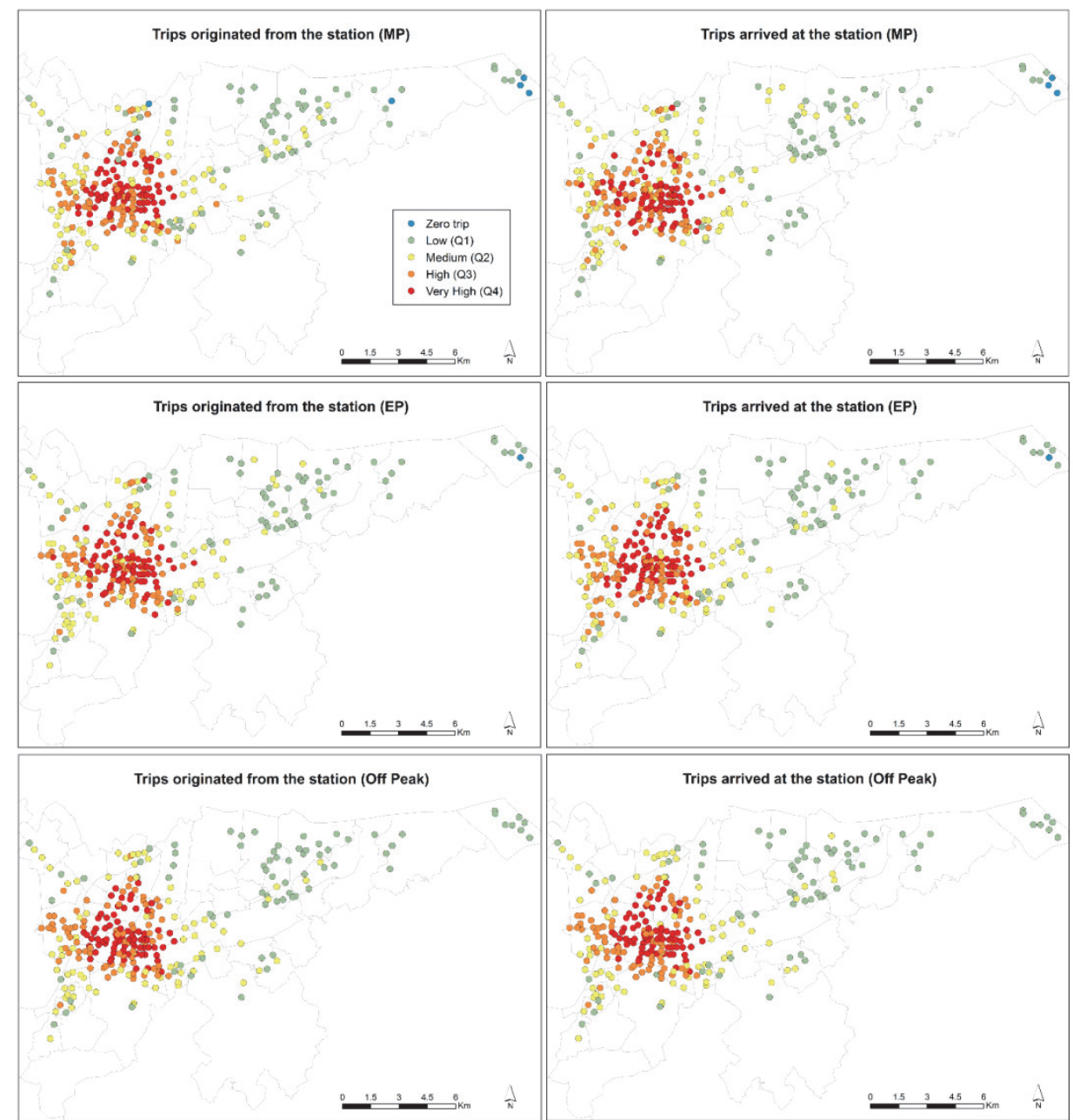

Figure 26 Bike-sharing trips originated from and arrived at the station.

\subsubsection{Research design}

The objective of this study is to investigate the travel behaviour of bike-sharing usage based on the trip data. This study was conducted for a bike-sharing system in Zhongshan (China), using 5-months of trip data from February 2014 to June 2014. In this paper, we took travel on weekdays as an example.

As part of the initial data exploration, we visualized cycling trips across the urban area to identify whether there are travel patterns in large volumes of the trip database. Figure 5.5 displays the origin-destination (O-D) bicycle flows during morning peak hours and evening peak hours of weekdays. The way that we visualized the O-D bicycle flows is similar to the approach proposed by Beecham and Wood (2014). We used curves to describe the different directions 
of bike flows between two stations, and emphasized the flow magnitude based on the color and thickness of flow lines. The thickness of flow lines is in proportion to a weighting that depends on the relative frequency of the trips: the ratio of the frequency of trips from a given O-D pair to the maximum frequency between any O-D pairs. According to Figure 27, in this study, the spatial distribution of O-D bicycle flows has several characteristics: (1) bicycle flows are divergent flows from/to a station; (2) there are large number of short-distance flows at local scale (TAZ-level); (3) the vast majority of lone flows are thin and crossing with each other; (4) there is a high density of stations in the central areas that generated large amount of trips. In short, the display of the O-D trips eclipses the patterns underneath, the information about the travel patterns and the driving force underlying the bicycle flows cannot be observed explicitly from the current visualization of O-D bicycle flows, i.e. "when, where, and why" those trips occurred.

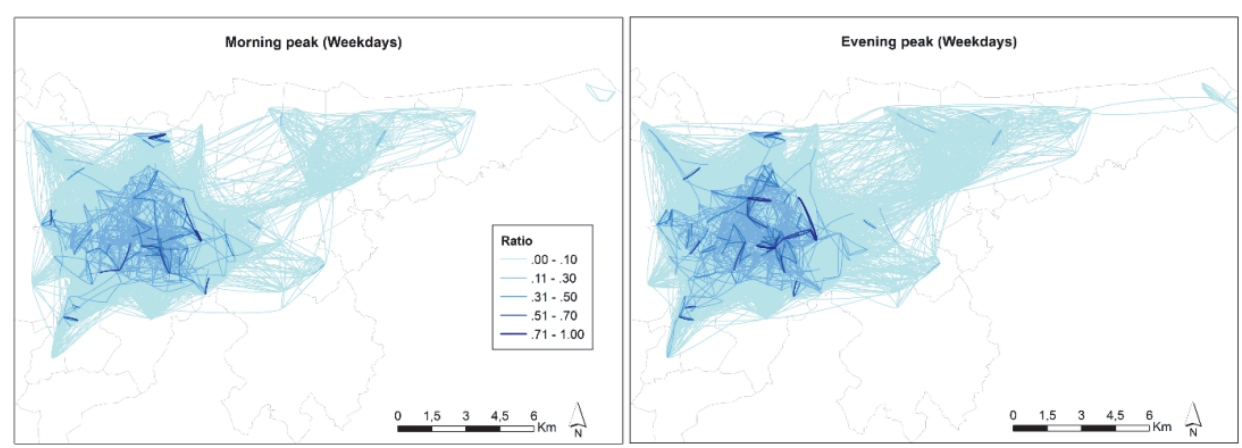

Figure 27 Origin-Destination bicycle flows during morning peak and evening peak hours of weekdays.

Within this context, to elucidate the travel patterns of bike-sharing usage, we decided to examine and synthesize three aspects: (1) bike-sharing trips; (2) trip chains; and (3) transition activities.

Table 13 introduces and explains the terminology used in this study. Moreover, we examined both spatial and temporal characteristics of individual trips, trip chains, and transition activities. The spatial characteristic relates to the land use type of each bike station, as bike-sharing trips vary based on land uses (Mateo-Babiano et al., 2016). The temporal characteristic refers to the time information relating to the start and end of individual trips, trip chains, and transition activities. 
Table 13 Definition and explanations of the terms used in this study.

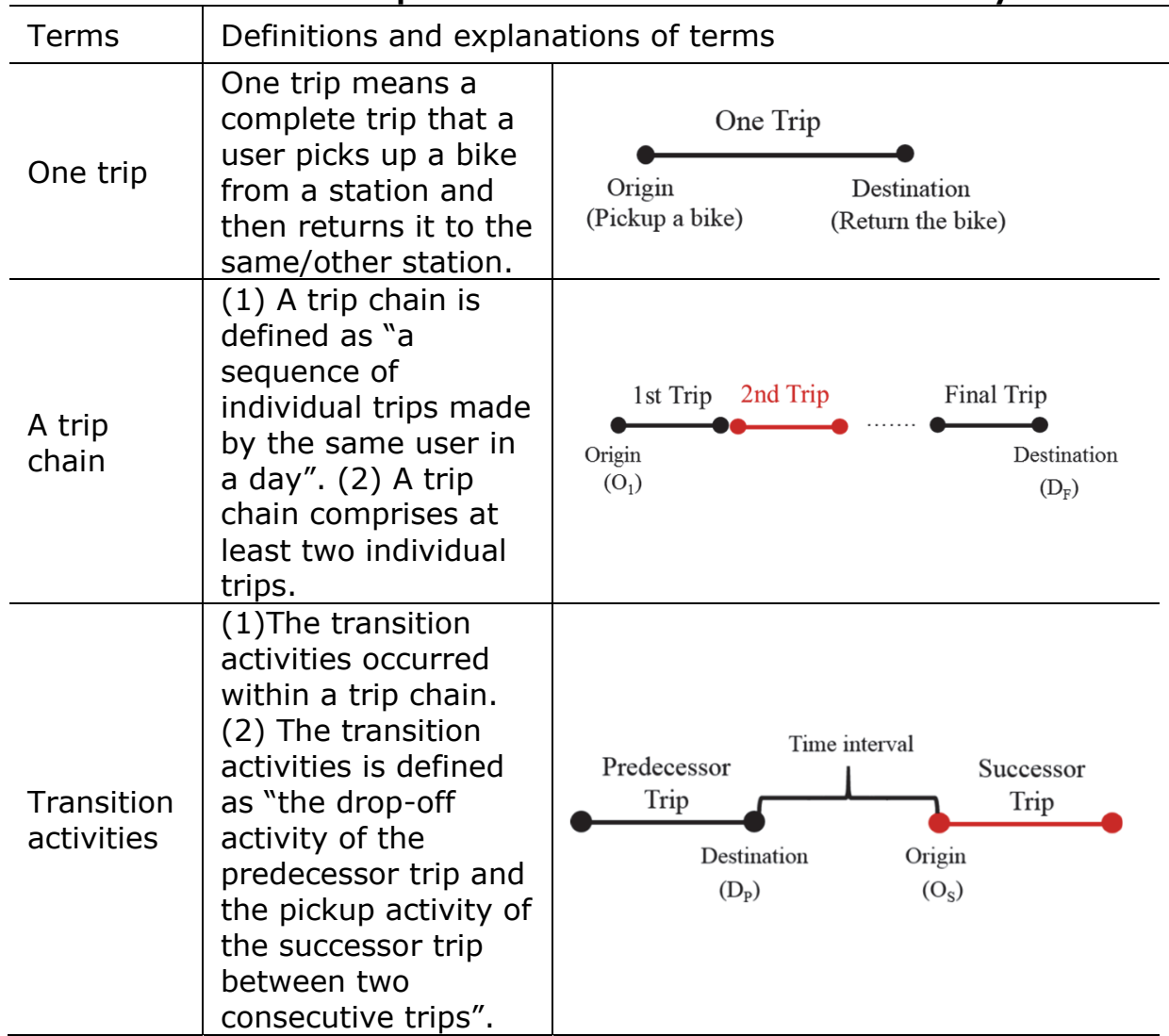

To examine the patterns of bike-sharing trips, we developed an OriginDestination (O-D) matrix to reveal when and where individual bike-sharing trips start and end. To investigate the patterns of trip chains, we also developed an O-D matrix to uncover when and where the first trip starts (i.e. origin) and the final trip ends (i.e. destination) of each trip chain. To examine the transition activities, we developed a transition matrix to disclose when and where each user completes one trip and starts again the next trip within a trip chain. The combination of O-D and transition matrices is an indication of the travel patterns and potential travel purpose of bike-sharing usage. The creation of individual trip chain was conducted in Python, and the spatial and statistical analyses were operated in ArcGIS and SPSS. The approach of constructing O$D$ and transition matrices was inspired by Wu et al. (2014) who explored human mobility and activity based on social media check-in data.

According to Eq. 5.1, we constructed an O-D matrix of individual trips, i.e. MT. $S=\{s 1, s 2, \ldots$.$\} to denote the dominant land use type of each bike station.$ In our study, the dominant land use type of each station was defined based on 
the principle of site-selection of bike stations: bike stations are usually named after the nearby residential community, shopping mall, park, institution, etc. As a result, bike stations are classified into five land use types (as shown in Figure 28): residential, commercial, institution (education, bank, government offices), recreational (park, cinema), and transportation (bus stop, train station). $T=\{t 1, t 2, \ldots\}$ denotes the start time and end time of each trip. The size of $\mathrm{M}(\mathrm{O}-\mathrm{D})$ is $\mathrm{N} \times \mathrm{N}$ with $\mathrm{N}=|\mathrm{S}| \times|\mathrm{T}|$. The cell of $\mathrm{M}$ with index $\left(S_{p}^{t_{i}}, S_{q}^{t_{j}}\right)$, where $p$ and $q \in[1,|S|]$ and $i$ and $j \in[1,|T|]$, records the frequency of trips that originated from a station relating to land use type $p$ at time $t_{i}$ and arrived at a station relating to land use type $q$ at time $t_{j}$.

$$
M_{T}\left(p_{i}, q_{j}\right)=\left\{(x, y) \mid \mathrm{x}=S_{p}^{t_{i}}, y=S_{q}^{t_{j}}\right\}
$$

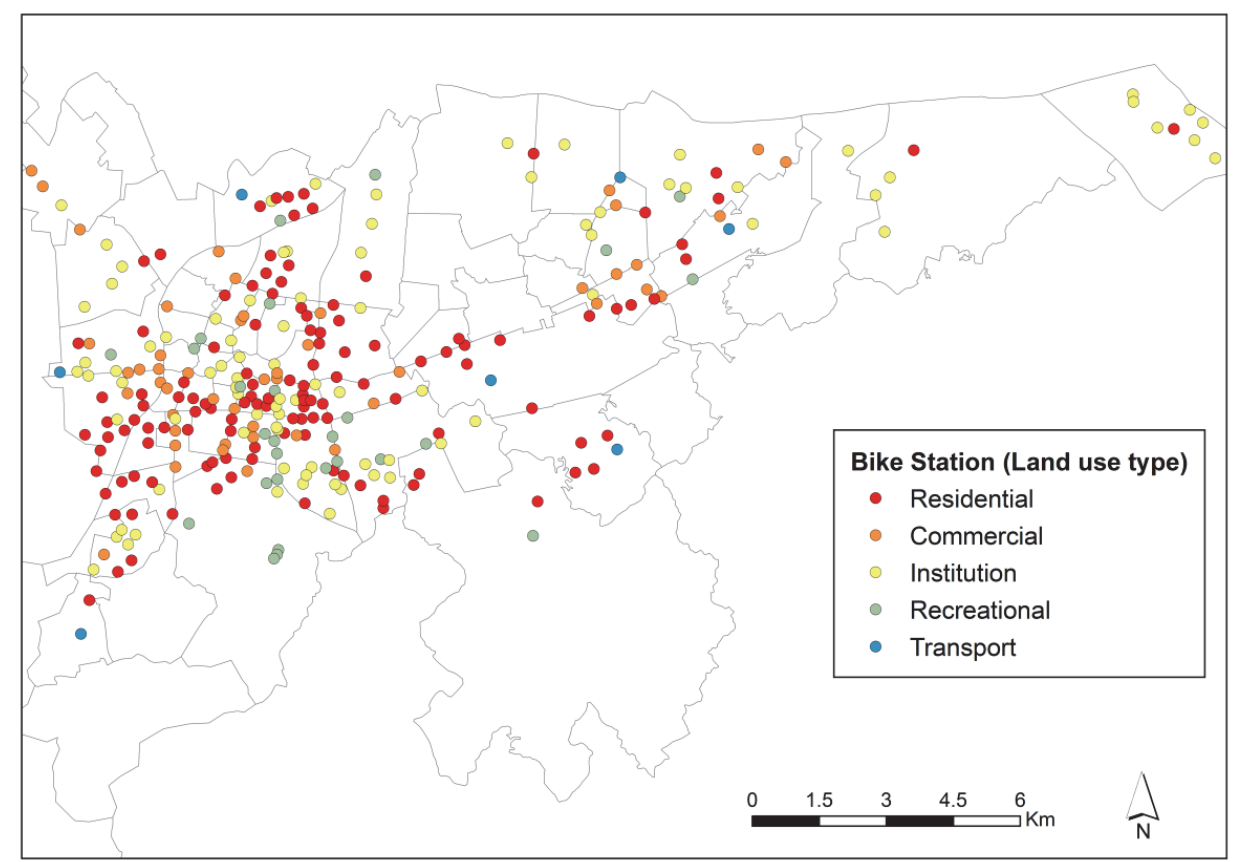

Figure 28 Dominant land use types of bike stations.

Eq. 5.2 describes the O-D matrix of individual trip chains, i.e. MTc. Similar to Equation $5.1, S=\{s 1, s 2, \ldots$.$\} denotes the five types of land use type related$ to bike stations. The column of MTc represents the pickup activity of the first trip, and the row of $\mathrm{M}_{\mathrm{TC}}$ represents the return activity of the final trip. The cell of MTc with index $\left(S_{f}^{t_{i}}, S_{l}^{t_{j}}\right)$, where $f$ and $l \in[1,|S|]$ and $i$ and $j \in[1,|T|]$, records the number of trip chains that the first trip originated from stations relating to land use type $f$ at time $t_{i}$, and the final trip arrived at stations relating to land use type $l$ at time $t_{j}$. 


$$
M_{T C}\left(f_{i^{\prime}} l_{j}\right)=\left\{(x, y) \mid x=S_{f}^{t_{i}}, y=S_{l}^{t_{j}}\right\}
$$

According to Eq. 5.3, we constructed a transition matrix for transition activities, i.e. $\mathrm{M}_{\mathrm{TA}}$. Similar to Eq. $5.1, \mathrm{~S}=\{\mathrm{s} 1, \mathrm{~s} 2, \ldots$.$\} denote the five types of land use$ type related to bike stations. The column of MTA represents the arrival activity of last trip, and the row of $\mathrm{M}_{\mathrm{TA}}$ represents the departure activity of the next trip. The cell of MTA with index $\left(S_{m}^{t_{i}}, S_{n}^{t_{j}}\right)$, where $m$ and $n \in[1,|S|]$ and $i$ and $j \in$ $[1,|T|]$, records the frequency of occurrences that a user complete a trip at a station relating to land use type $m$ at time $t_{i}$ and starts the next trip at a station relating to land use type $n$ at time $t_{j}$.

$$
M_{T}\left(m_{i}, n_{j}\right)=\left\{(x, y) \mid \mathrm{x}=S_{m}^{t_{i}}, y=S_{n}^{t_{j}}\right\}
$$

Consequently, each matrix has 25 large cells $(5$ rows $\times 5$ columns, representing five land use types), and each large cell is comprised of 576 small cells ( 24 rows $\times 24$ columns, representing 24 hours). In addition, when visualizing O-D and transition matrices, we used the Natural Breaks (Jenks) classification method to break the class of values, because this class breaks method identifies the best group similar values and maximizes the differences between classes.

\subsection{Analysis and Results}

\subsubsection{Types of trips, trip chains, and transition activities}

Table 14 describes the classification of trip data and types of bike-sharing usage. The definition of trip data is based on the condition that individual users make either one trip (Type I) or more than one trip (Type II) in a day. This lays the foundation for exploring trip chains and transition activities. Moreover, individual trips are classified into two categories: (1) loop trips, i.e. the origin and destination of a trip are the same station; and (2) directional trips, i.e. the origin and destination of a trip are different stations. As shown in Table 14, Type II accounts for $68 \%$ of weekday trips. Moreover, compared to loop trips, directional trips make up the vast majority of trips, for both Type I and Type II. This indicates that the majority of bicycle trips travelled from a station to the other station. The occurrence of loop trips might be associated with recreational activities (physical exercises), or short-time activities (e.g. quick shopping) (Bordagaray et al., 2016). 
Table 14 Types of individual bike-sharing trips (weekdays).

\begin{tabular}{lll}
\hline & & Percentage \\
\hline Type I & Trip data (Users make one trip in a day) & $32 \%$ \\
& Loop trips & $2.5 \%$ \\
& Directional trips & $29.5 \%$ \\
\hline \multirow{2}{*}{ Type II } & Trip data (Users make more than one trip in a & $68 \%$ \\
& day) & $2.3 \%$ \\
& Loop trips & $65.7 \%$ \\
\hline
\end{tabular}

Based on the trip data that users make more than one trip in a day (i.e. Type II ), Table 15 describes the types of individual trip chains and transition activities. Based on the origin station of the first trip and the destination station of the final trip in each trip chain, the trip chains are classified into two categories: (1) loop trip chains (Type II -A), i.e. $\mathrm{O}_{1}$ and $\mathrm{D}_{\mathrm{F}}$ are the same station (see the explanation in Table 1); and (2) non-loop trip chains (Type II-B), i.e. $\mathrm{O}_{1}$ and $\mathrm{DF}_{\mathrm{F}}$ are the different stations (see the explanation in Table 13). As show in Table $15,51 \%$ of trip chains belong to loop trip chains, i.e. each user originates from a station for the first trip and arrives at the same station for the final trip in a day, and $48 \%$ of trip chains are the non-loop trip chains. The loop trip chains might be connected with commuting activities. The occurrence of non-loop trip chains can be explained by two possibilities: firstly, the $\mathrm{O}_{1}$ and $D_{F}$ are close to each other, the $\mathrm{O}_{1}$ station is full so that users have to choose a nearby station $\left(D_{F}\right)$ to drop off the bike. In this case, the non-loop trip chain is the potential loop trip chain, which is result of the station capacity. Secondly, station $\mathrm{O}_{1}$ and station $\mathrm{D}_{\mathrm{F}}$ are far from each other, users either are not back or choose other travel modes to go back to the origin of the first trip. To this end, we examined the network distance between the origin station of the first trip $\left(\mathrm{O}_{1}\right)$ and the destination station of the final trip ( $\left.D_{F}\right)$. According to Figure 5.8, around $50 \%$ of the non-loop trip chains show that the network distance between station $\mathrm{O}_{1}$ and station $\mathrm{D}_{\mathrm{F}}$ is less than $600 \mathrm{~m}$, and the majority of the trip chains show that the network distance between station $\mathrm{O}_{1}$ and station $\mathrm{DF}_{\mathrm{F}}$ is less than $1000 \mathrm{~m}$. In general, for the majority of non-loop trip chains (Type II -B), the network distance between the origin station of the first trip and the destination station of the final trip is not far from each other. 
Table 15 Types of transition activities and trip chains (weekdays).

\begin{tabular}{|c|c|c|c|}
\hline Trip data (Type II ) & & & Percentage \\
\hline \multirow[t]{2}{*}{$\begin{array}{l}\text { Types of trip } \\
\text { chains }\end{array}$} & Type II -A & Loop trip chains $\left(\mathrm{O}_{1}=\mathrm{D}_{\mathrm{F}}\right)^{*}$ & $51 \%$ \\
\hline & Type II -B & $\begin{array}{l}\text { Non-loop trip chains } \\
\left(\mathrm{O}_{\left.1 \neq \mathrm{D}_{\mathrm{F}}\right)^{*}}\right.\end{array}$ & $49 \%$ \\
\hline \multirow[t]{2}{*}{$\begin{array}{l}\text { Types of } \\
\text { transition } \\
\text { activities }\end{array}$} & Type II -C & $\begin{array}{l}\text { Transfer at the same station } \\
\left(D_{p}=O_{s}\right)^{*}\end{array}$ & $65.9 \%$ \\
\hline & Type II -D & $\begin{array}{l}\text { Transfer at different stations } \\
\left(\mathrm{D}_{\mathrm{p} \neq \mathrm{O}}\right)^{*}\end{array}$ & $34.1 \%$ \\
\hline
\end{tabular}

* O $\mathrm{O}_{1}, \mathrm{D}_{\mathrm{F}}, \mathrm{Dp}_{\mathrm{p}}, \mathrm{Os}_{\mathrm{s}}$ as shown in Table 13

According to Table 15, the transition activities are categorized into two groups: (1) transfer at the same station (Type II-C), i.e. a user arrives at a station when completing a trip and departs from the same station when starting the next trip ( $D_{p}=O_{s}$ in Table 5.1); and (2) transfer at different stations (Type II -D, i.e. $D_{p} \neq O_{s}$ in Table 5.1). As shown in Table 15, the majority of transition activities belong to Type II -C, i.e. transfer at the same station. Activities that transfer at different stations (Type II -D) can be explained by two possibilities: firstly, station $D_{p}$ and station Os are close to each other: station $D_{p}$ is empty so that users have to choose a nearby station (Os) to pick up a bike when starting the successor trip; secondly, station $D_{p}$ and station $O_{s}$ are far from each other: between two consecutive trips, users moved to the other place. To this end, we examined the network distance between the destination station of the predecessor trip $\left(D_{p}\right)$ and the origin station of the successor trip $\left(\mathrm{O}_{s}\right)$, and also took account of the interval time between two consecutive trips. As shown in Figure 30, it does not matter if the interval time is less or more than 1 hour, the majority of the transition activities (Type II -D) show that the network distance between station $D_{p}$ and station $O_{s}$ is less than $600 \mathrm{~m}$. Moreover, the vast majority of transition activities (Type II -D) where the interval time is less than an hour also show that the network distance between two stations is less than $600 \mathrm{~m}$. This implies that majority of the activities that transfer at different stations might be attributed to the fact that the station capacity is empty so that users have to shift to a nearby station to rent a bike. This also suggests that neighbouring stations are complementary to each other. 


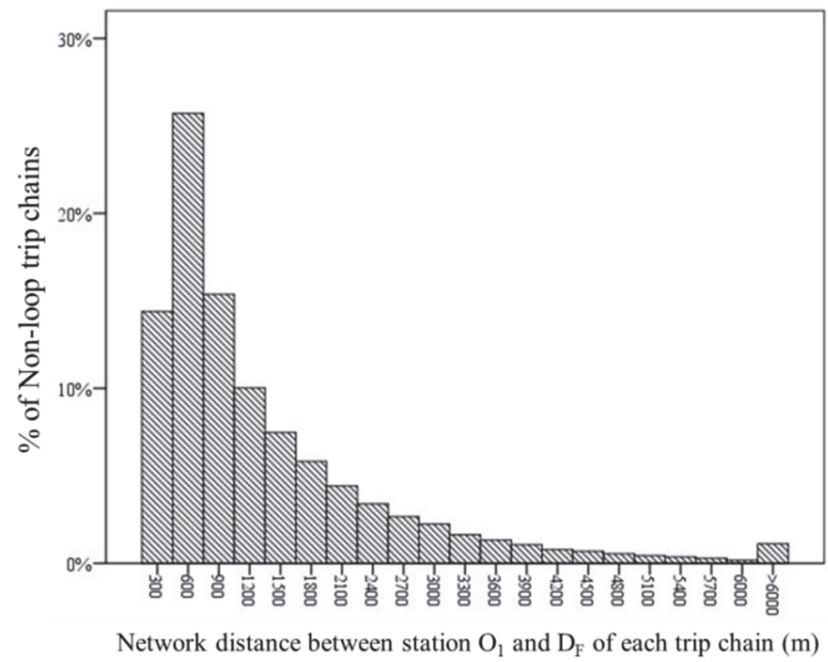

Figure 29 The network distance between station $O_{1}$ and station $D_{F}$ of non-loop trip chains on weekdays.

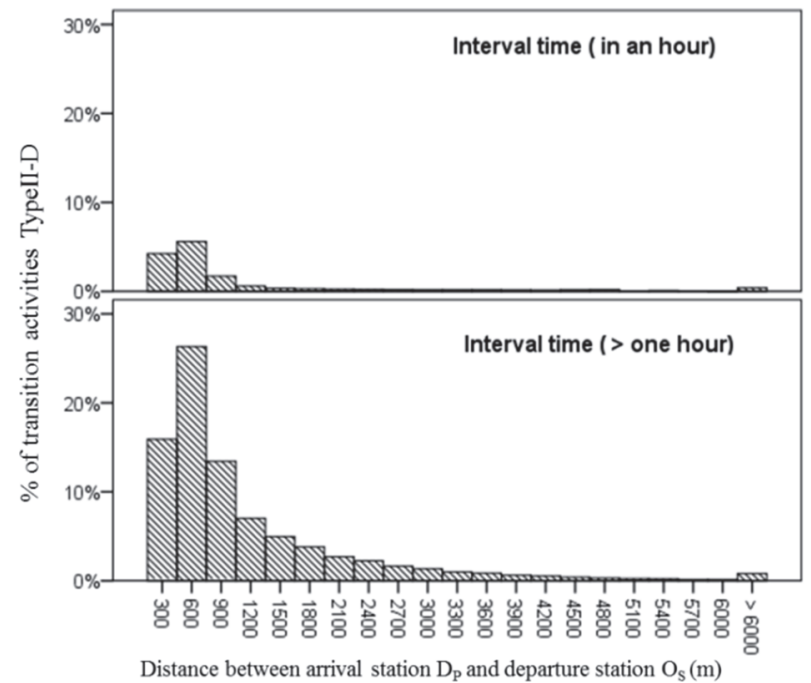

Figure 30 Distribution of the network distance between transfer stations $D_{p}$ and Os.

\subsubsection{Patterns of bike-sharing usage}

In this section, we investigate and explain the patterns of bike-sharing usage in three aspects: individual bike-sharing trips, trip chains, and transition activities. The synthesis of those three aspects indicates the patterns of bikesharing usage. To clarify the visualization results of O-D and transition matrices, we zoomed in one of the large cells as an example. 


\section{- Patterns of bike-sharing trips}

Figure 31 and Figure 32 describe the O-D matrices of individual trips, for trip data Type I (i.e. trip data that users make one trip in a day) and Type II (i.e. trip data that users make more than one trip in a day) respectively. Each cell represents the percentage of trips that depart from and arrive at where (i.e. the land use type of each station) and at what time (i.e. 24 hours of the day).

As shown in Figure 31 and Figure 32, the majority of trips occurred between stations near residential, commercial, and institutional areas. This mainly attributes to the fact that the vast majority of stations are located near residential, commercial, and institutional areas, as shown in Figure 28. Moreover, two primary trip patterns can be observed for both Type I and Type II : first, morning peak hours, i.e. bike-sharing trips both start and end at 7-8 (or 8-9) a.m. (marked as area A); second, evening peak hours, i.e. bikesharing trips both start and end at 5-6 (or 6-7) p.m. (marked as area B). In addition, a distinctive pattern observed for Type II : Lunch peak hours, i.e. trips start at 12-2 p.m. and end in an hour (marked as area C). The observed patterns of bike-sharing trips imply that the majority of bike-sharing trips might relate to work, home, and shopping.

As regards the departure and arrival stations of each trip, there are a number of trips travelling between two stations with the same land use types (especially the residential area), the reason for this is that we only consider the dominant land use type of the station. In reality, there is high density of mixed land use patterns in the study area. For example, the dominant land use type of a station is residential area (i.e. the station is located in front of the gate of the residential community), but there are many retail shops and groceries that belong to the residential community along the street, which are also near the bike station; the retailers and grocers are also potential users that use public bikes for commuting or running errands. 


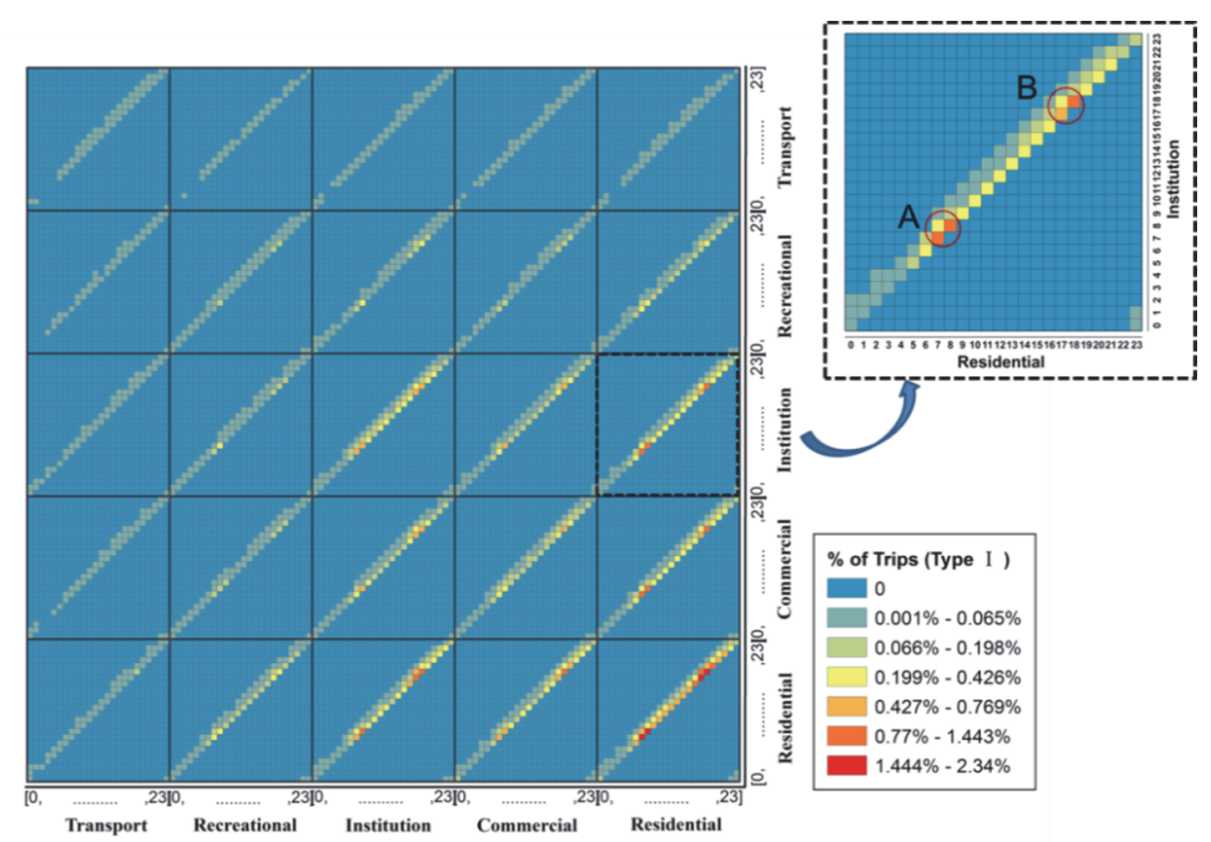

* $X$ represents the origin time and locations and $Y$ represents the destination time and locations.

Figure 31 O-D matrix of individual trips (Type I ) on weekdays.

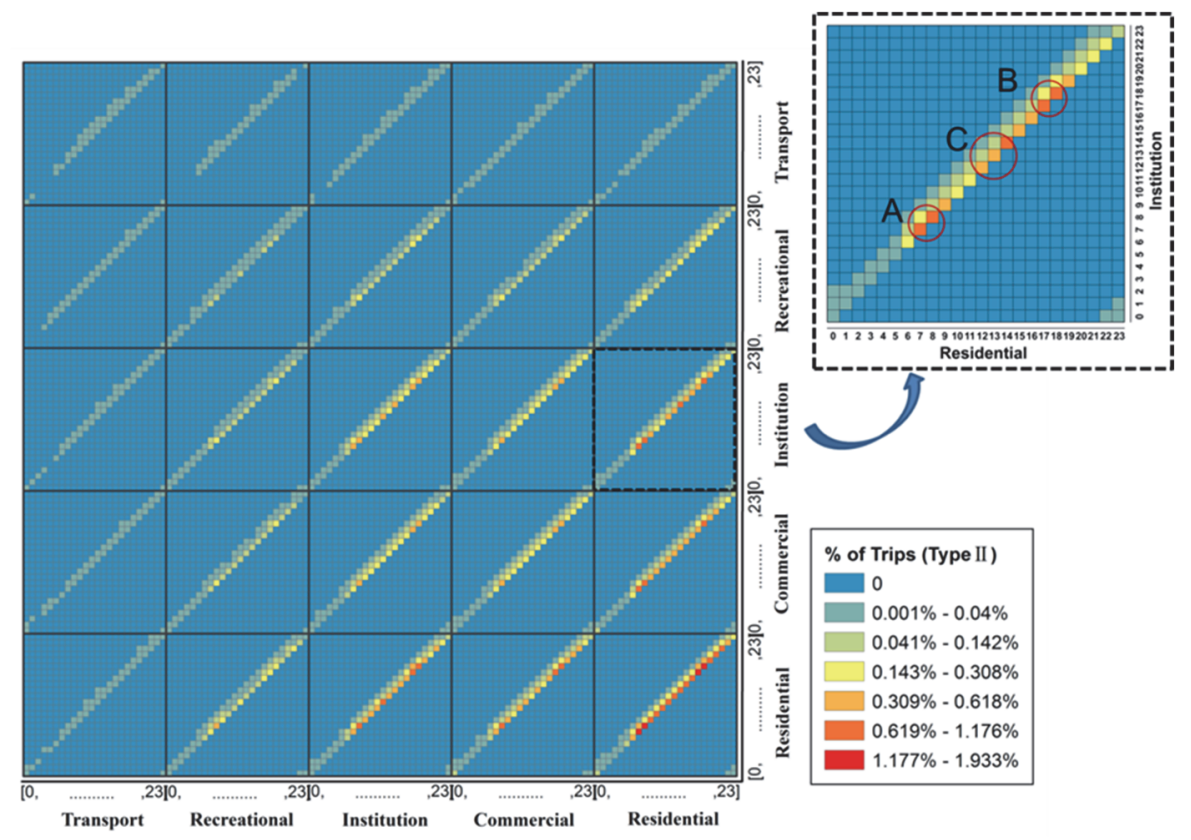

*X represents the origin time and locations and $Y$ represents the destination time and locations.

Figure 32 O-D matrix of individual trips (Type II ) on weekdays. 


\section{- Patterns of trip chains}

Figure 33 and Figure 34 describe the O-D matrices of individual trip chains, for Type II -A (i.e. Loop trip chains) and Type II-B (i.e. Non-loop trip chains) respectively. Each cell represents the percentage of trip chains: when and where the first trip starts and the final trip ends within a trip chain.

As shown in Figure 33 and Figure 34, a predominant pattern is observed for both Type II -A and Type II-B: the first trip starts at 7-8 or 8-9 a.m., and the final trip ends at 5-6 or 6-7 p.m. (marked as area D). This accounts for the largest number of trip chains of both Type II -A and Type II -B, and implies that the majority of bike-sharing usages might relate to commuting. To further confirm the patterns of trip chains belonging to area $D$ of Type II - $B$, we examined the network distance between station $\mathrm{O}_{1}$ and station $\mathrm{D}_{\mathrm{F}}$ of Type II -B trip chains, in order to understand whether this is the potential loop trip chain. As shown in Figure 35, around $60 \%$ and $75 \%$ of trip chains show that the network distance between $\mathrm{O}_{1}$ and $\mathrm{D}_{\mathrm{F}}$ is less than $600 \mathrm{~m}$ and $900 \mathrm{~m}$ respectively. This suggests that for the predominant pattern (marked as area D) of Type II $B$, the majority of trip chains are the potential Type II -A (loop trip chains). This can be explained by two possibilities: first, the $\mathrm{O}_{1}$ is full so that users have to choose a nearby station $\left(D_{F}\right)$ to return the bike. Second, two stations are close to each other, users just select one of them randomly.

In addition, several additional patterns can also be observed, although these patterns only account for a small proportion of trip chains. Type II -A (loop trip chains) shows two distinctive patterns: first, the first trip starts at 8-9 a.m. and the final trip ends at 12-1 p.m. (marked as area E in Figure 33); second, the first trip starts at 2-3 p.m. and the final trip ends at 5-6 p.m. (marked as area $\mathrm{F}$ in Figure 33). Type II -B (non-loop trip chains) also shows two distinctive patterns: first, the first trip starts at 7-9 a.m. and the final trip ends at 1-3 p.m. (marked as area G in Figure 34); second, the first trip starts at 11 a.m.12 p.m. and the final trip ends at 5-6 p.m. (marked as area $\mathrm{H}$ in Figure 34). Although those patterns are observed for different types of trip chains, they have features in common: the patterns are primarily for the first trip departures from and the final trip arrivals at stations near residential and institutional areas. Moreover, those trip chains only occur in a half daytime, either from morning to noon or from noon to evening. This implies that bikesharing usage might only occupy parts of the commuting activities. 


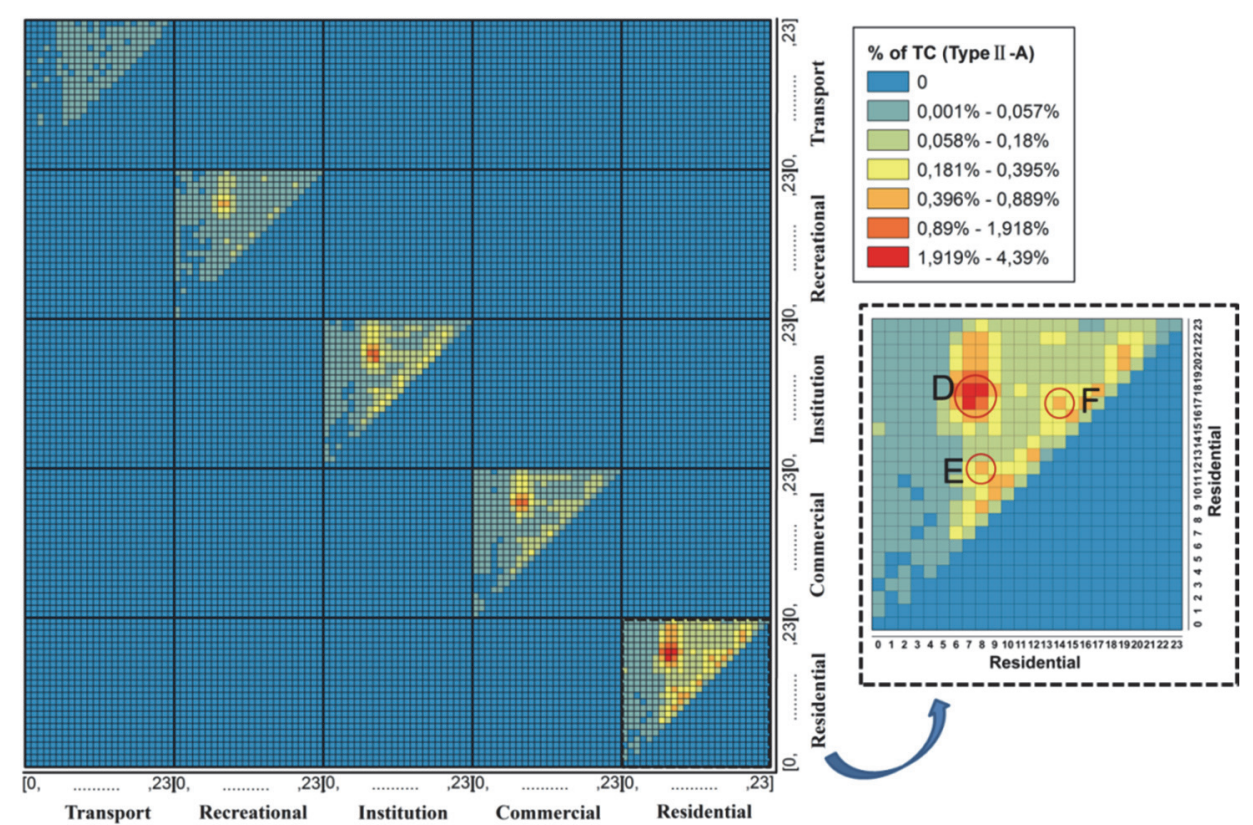

*X represents the start time and location of the first trip, and $Y$ represents the arrival time and location of the final trip.

Figure 33 O-D matrix of trip chains (Type II-A) on weekdays.

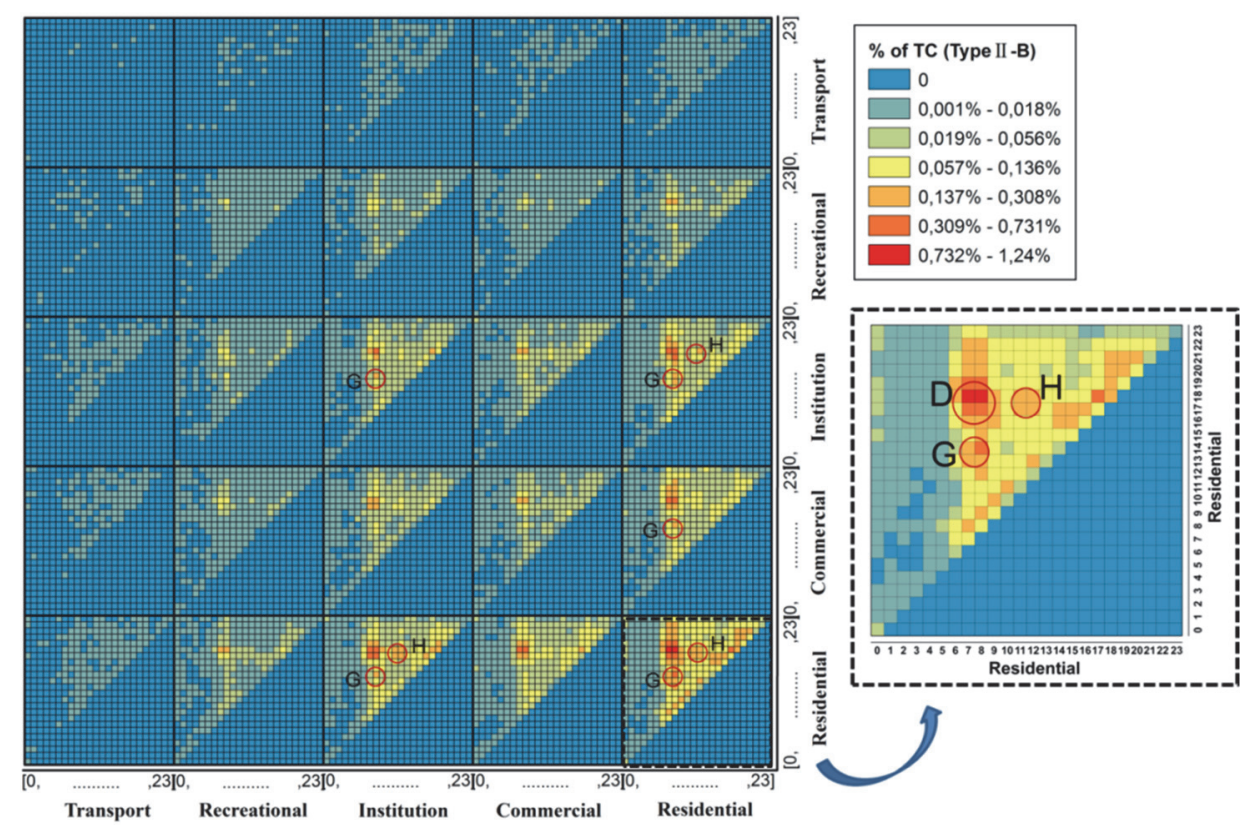

*X represents the start time and location of the first trip, and $Y$ represents the arrival time and location of the final trip.

Figure 34 O-D matrix of trip chains (Type II-B) on weekdays. 


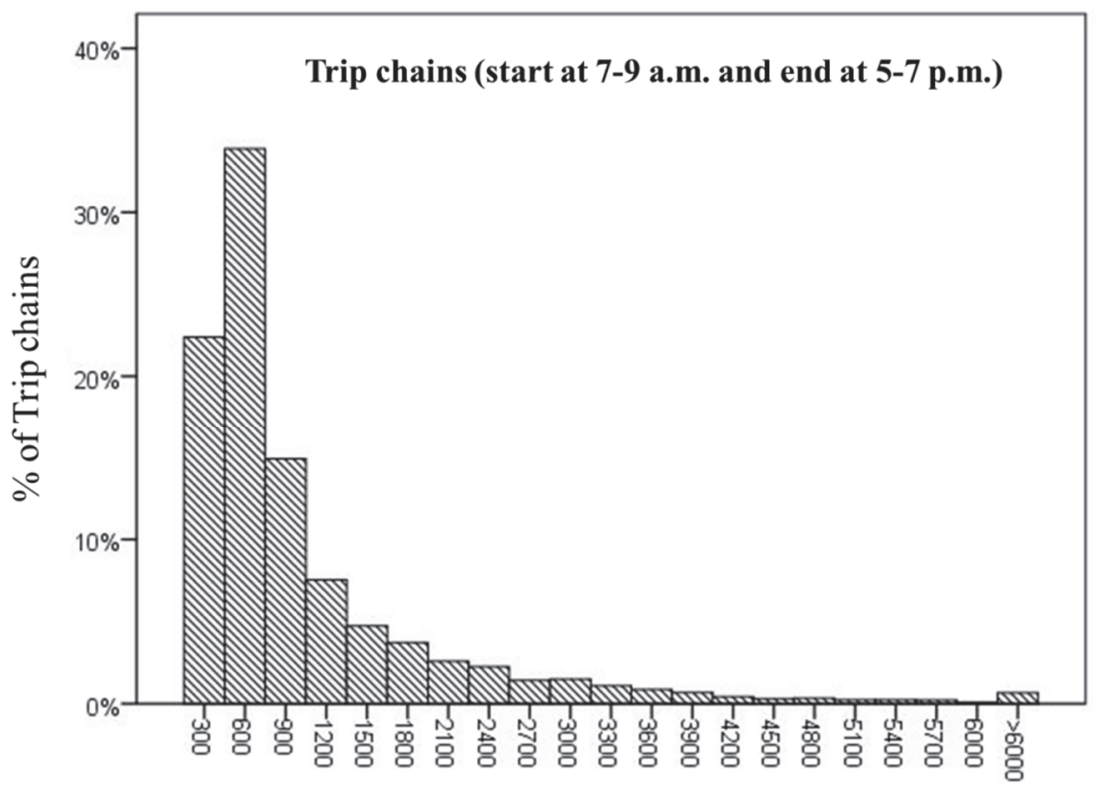

Network distance between station $\mathrm{O}_{1}$ and $\mathrm{D}_{\mathrm{F}}$ of each trip chain $(\mathrm{m})$

Figure 35 Network distance between the start and end stations of non-loop trip chains (7-9 a.m. 5-7 p.m.).

\section{- Patterns of transition activities}

Figure 36 and Figure 37 show the matrices of transition activities between two consecutive trips, for Type II -C and Type II -D respectively. Each cell represents the percentage of transition activities: when and where the preceding trip ends and the next trip starts in a day.

As shown in Figure 36 and Figure 37, four predominant patterns can be observed for both Type II -C and Type II -D: (1) users completed a trip at 7-9 a.m. and started the next trip at 5-7 p.m. (marked as area I); (2) users completed a trip at 8-9 a.m. and started the next trip at 12 p.m. (marked as area $\mathrm{J}$ ); (3) users completed a trip at 12 p.m. and started the next trip at 122 p.m. (marked as area $\mathrm{K}$ ); and (4) users completed a trip at 1-2 p.m. and started the next trip at 5-7 p.m. (marked as area L). Those transition patterns suggest users might use public bikes for going to work and home. Moreover, referring to Type II $-\mathrm{A}$, there are some cases showing that users completed a trip at 5-6 p.m. and started again at the same station, and the interval time is less than one hour. In this case, for the stations nearby shopping mall, the transition activities might be attributed to the after-work shopping, and for stations nearby institutions and residential communities, the transition activities might be attributed to running errands. 


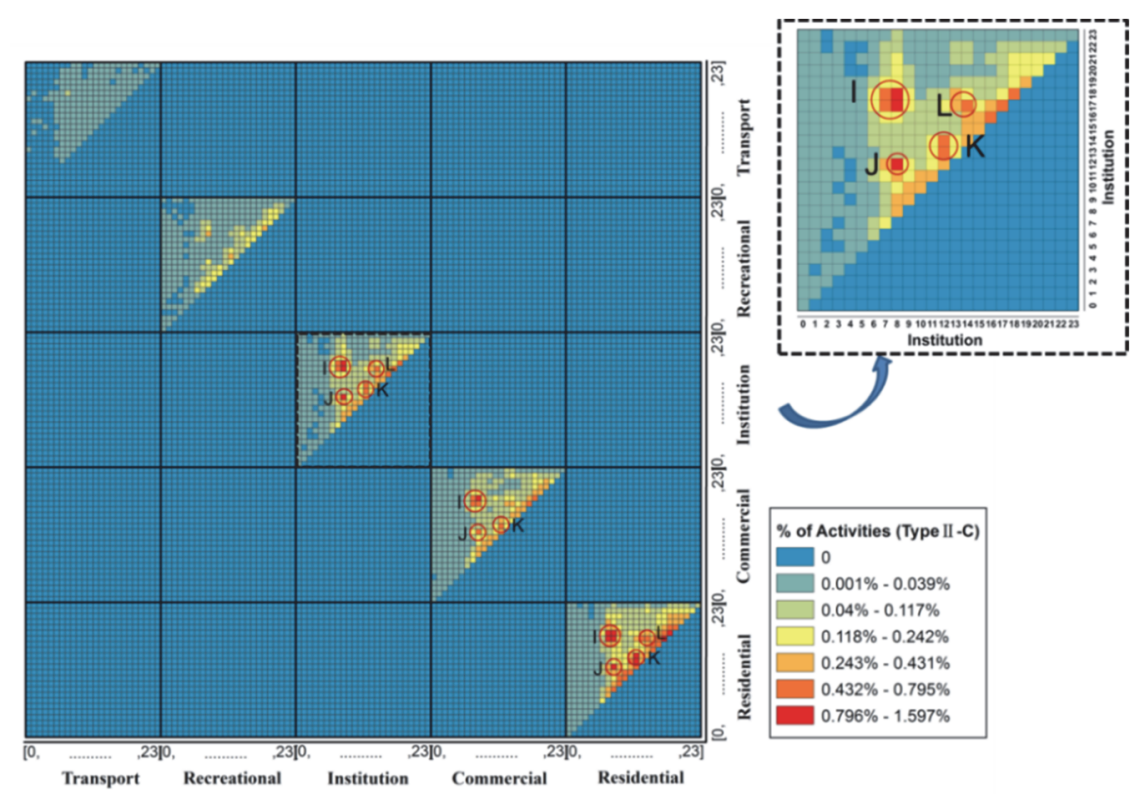

$* X$ represents the arrival time and location of the predecessor trip, and $Y$ represents the departure time and location of the successor trip.

Figure 36 Transition matrix of bike-sharing usage (Type II-C) on weekdays.

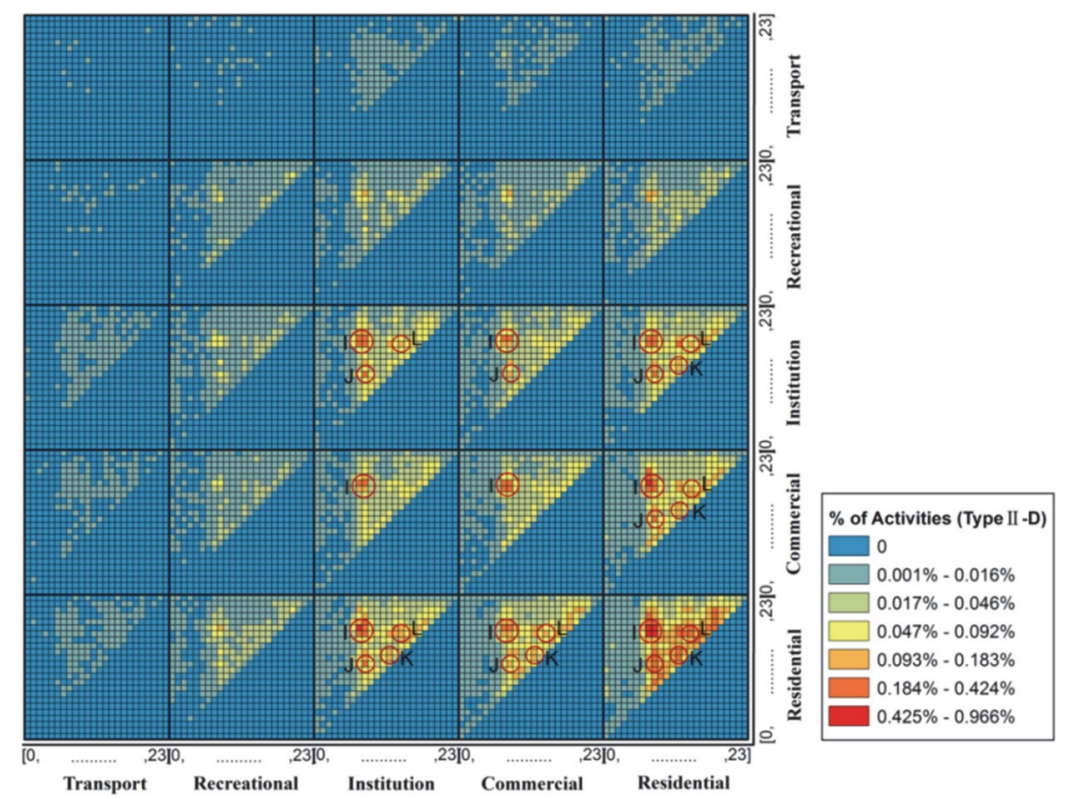

$X$ represents the arrival time and location of the predecessor trip, and $Y$ represents the departure time and location of the successor trip.

Figure 37 Transition matrix of bike-sharing usage (Type II -D) on weekdays. 


\subsection{Conclusions}

There is some peer-reviewed literature on travel behaviour of bike-sharing usage, i.e. patterns of bike-sharing trips. However, these studies mainly focused on the movement (trips) of bike-sharing usage and do not pay attention to the transition activities between consecutive bike-sharing trips within a trip chain. Moreover, most such studies aim at emphasizing the strong origin-destination pairs but fail to incorporate the information of land use type and time that relates to the start and end of individual trips. To illustrate the travel behaviour of bike-sharing usage, this paper explored the patterns of bike-sharing usage in three aspects: bike-sharing trips, trip chains, and transition activities, using 5 months of trip data from a bike-sharing system in Zhongshan, China.

One of the highlights of this paper is the incorporation and synthesis of individual trips, trip chains, and the transition activities to explore the travel behaviour of bike-sharing users. Moreover, we also considered the time and land use information relating to the start and end of individual trips, trip chains, and transition activities. As a result, we constructed Origin-Destination matrices and transition matrices, incorporating the hourly pickup and drop-off activities and land use type of each station, to uncover the patterns and potential purpose of bike-sharing usage.

Based on the classifications of individual trips, trip chains, and transition activities, the statistic results indicate that only $4.8 \%$ of trips are loop trips that start and end at the same station, which might be associated with recreational activities (physical exercises), or short-time activities (e.g. quick shopping) (Bordagaray et al., 2016). 51\% of trip chains belong to loop trip chains, which might be connected with commuting activities. Although $49 \%$ of trip chains are non-loop trip chains, most trip chains show that the network distance between the origin of the first trip and the destination of the final trip is less than $600 \mathrm{~m}$. This might be attributed to two possibilities: first, the origin station is full so that users have to choose a nearby station (destination station) to return the bike. Second, two stations are close to each other (acceptable walking distance), and users just select one of them at random. As regards the transition activities, $66 \%$ of transition activities show transfer at the same station. Based on the network distance between stations, the findings also suggest that transferring at different stations might be attributed to the fact that the station capacity is empty so that users have to choose a nearby station to find a bike. This also suggests that neighbouring stations might be complementary to each other.

Findings from The Origin-Destination matrices of individual trips indicate three dominant patterns: morning peak hours (trips both start and end at 7-9 a.m.), evening peak hours (trips both start and end at 5-7 p.m.), and lunch peak 
hours (trips both start and end at 12-1p.m.). Moreover, the vast majority of trips occur between stations near residential, commercial, and institutional area, due to the site-selection of bike stations. Findings from the O-D matrices of trip chains indicate a predominant pattern: the first trip starts at 7-8 or 8-9 a.m., and the final trip ends at 5-6 or 6-7 p.m. This accounts for the largest number of trip chains, and suggests that the majority of bike-sharing usages might relate to commuting. According to the transition matrices, three primary patterns are identified: (1) the interval time is around 10-11 hours (i.e. arrive at 7-9 a.m. and start again at 5-7 p.m.); (2) the interval time is around 3-4 hours (i.e. arrive at 7-9 a.m. and start again at 12 p.m.); and (3) the interval time is 1-2 hours (i.e. arrive at 12 p.m. and start again at 2p.m.). In general, those findings suggest that users mainly used public bikes for commuting, and some of users went home during lunch break. Moreover, some of users show an after-work shopping activity.

This study provides a complete insight into how the bike-sharing system is used, in terms of both trips and activities. With reference to those methods and insights, urban planners, policy makers and researchers can also explore the patterns of trip chains and transition activities of other systems, which could be beneficial to improving the existing system. 
Chapter 6

Synthesis 
Bike-sharing is booming. With the popularity of introducing bike-sharing programs all over the world, researchers have shown a huge interest in studying transport supply and travel demand aspects of these systems. The peer-reviewed literature on bike-sharing systems has been discussed in detail in each of the previous chapters. Despite of the interesting findings and the implications of these studies, yet a number of important research gaps can be identified, of which four are mentioned in chapter 1 . From this perspective an empirical study has been conducted to investigate the actual use of a bikesharing system by extracting data from its operational database. The underlying aim was to develop approaches to acquire relevant transport planning data from the database and to explore possibilities and limitations of using such a database. For this purpose the bike-sharing system in Zhongshan, a medium sized Chinese city, has been employed for which temporal selections of the operational database were available.

To this end, four sub-objectives have been formulated: (1) to investigate the overall characteristics of bike-sharing use, in terms of system efficiency, trip characteristics, and station activity patterns; (2) to examine the effect of surrounding built environment factors on the use of public bikes at bike stations; (3) to explore the dynamics of the systems (in users and demands) across the urban area and over years; and (4) to explore the travel behaviour of bike-sharing users. Those four objectives relate one on one to chapters 2 to 5 respectively.

This last chapter summarizes the main findings of this research. The main conclusions and some policy implications with respect to the four subobjectives are presented in section 6.1 and some reflections on the research are provided in section 6.2. The last section contains recommendations for future research.

\subsection{Conclusions}

\subsubsection{Objective 1: To examine the overall characteristics of bike-sharing usage in terms of system efficiency, trip characteristics, and station activity patterns.}

In chapter 2 we found that the overall system is not very efficient in comparison with metrics derived from a global analysis by Gauthier et al. (2013). This finding is mainly attributed to the low bike use and low market penetration in peripheral zones. To improve system efficiency, an increase in station density in these areas, possibly combined with a reduction in station capacity, would be worthwhile to consider. 
Demand for public bikes is relatively high in central zones, where population density is the highest and urban activities are concentrated. However, there is no specific orientation of trips to or from the city centre. The majority of bikesharing trips are short distance trips within or between adjacent zones. This finding suggests that bike-sharing trips mainly substitute for walking trips rather than for car or public transport trips. Moreover, there is no clear direction of inbound or outbound trips in rush-hour. The short distance of bikesharing trips and the absence of a clear orientation might be attributed to two reasons: firstly, the high mix of land use types leading to the short travel distance; secondly, a lack of or not overall well developed bike infrastructure. Weekdays and weekends show similar patterns for many people in China commute both on weekdays and in weekends (O'Brien et al., 2014; Zhao et al., 2015).

Finally, two types of users can be distinguished based on the frequency of bike use, that is, "incidental users" and "regular users". From a policy perspective, simplifying the access procedure for "incidental users" might encourage more people to use the system and to improve the turnover of bike use. Lathia et al. (2012) has shown that the introduction of an easier access procedure has raised bike use of London's public bike system.

\subsubsection{Objective 2: To examine the impact of built environment factors on the usage of public bikes at stations.}

In chapter 3 we found that stations that are closer to the city centre and with a higher population density within a $300 \mathrm{~m}$ buffer generated larger demands as well as a larger ratio of demand to supply (D/S), which is a common finding (e.g. Daddio (2012)). As expected, users prefer to choose stations that cover more bike lanes and branch roads that offer a bike-friendly environment and are more accessible to local communities (same to the findings of Faghih-Imani et al. (2014)). Station capacity shows a positive impact on both daily and hourly demand at the station, implying that users show a preference for stations with a large capacity, in order to increase the chance of finding a bike or parking slot (El-Assi et al., 2015). Although the study area has mixed land use patterns, model results indicated that the larger the number of different land use types within the $300 \mathrm{~m}$ buffer, the larger demand (and D/S) generated at stations. This suggests that more diverse land use types might attract a larger number of users with different travel purposes than a single land use type. A larger turnover is found for stations nearby a residential community during weekends and off-peak of weekdays, and lower turnover and demand generated at stations nearby a park during morning and evening peaks of weekdays.

There might be competition between nearby stations, i.e. users shift from a station to a nearby station to pick up or drop off bikes, as the number of other 
stations within the $300 \mathrm{~m}$ buffer of a station negatively affects both demand and $\mathrm{D} / \mathrm{S}$ at the bike station. Moreover, the spatially lagged dependent variable also indicated the spatial correlation of BSS usage between nearby stations, i.e. demand at a station is positively correlated with demand from nearby stations. This can be explained by two potential reasons: first, nearby station share the same built environment factors that result in the high (or low) demand at these stations (Cervero et al., 2009); second, the spillover effect of demand at nearby stations, i.e. if a station is near its capacity (no available bikes or parking slots), users will shift from a station to nearby stations to pick up or drop off bikes (Rudloff and Lackner, 2014).

The model for demand-supply ratio during weekdays indicated a negative impact of station capacity that is 1.7 times larger than the negative impact of the number of other stations within the $300 \mathrm{~m}$ buffer. When keeping other variables constant, we found that adding a new station (with empty capacity) within a $300 \mathrm{~m}$ buffer of a current station to share the capacity of the current station can improve the D/S at the current station, i.e. relocating the capacity of a station to a new station within the $300 \mathrm{~m}$ buffer of the station. This suggests that increasing the density of stations with small or medium-sized capacity can enhance the turnover at stations, which will also extend the service area of bike stations and reduce travel distances.

In our study, the existing public transport facility does not show a significant influence on the BSS usage at stations, implying that users did not tend to transfer from public bikes to public bus stops (or the other way round) in general. This can mainly be attributed to the low local modal split and less attractive public bus system. This suggests that the significant role of the bikesharing system is not an intuitive feeder mode to the existing public transport system in our study, but serves as a single mode for users to complete the entire trips.

Although the guideline on building bike-sharing systems is quite the same all over the world at the present stage, there is a substantial necessity to understand the local travel modes before building bike-sharing systems, and define the role of bike-sharing systems in the context of cities rather than a priori treat such systems as a feeder mode to existing public transport systems, so that the system can be used efficiently.

\subsubsection{Objective 3: To examine the dynamics of the bike- sharing system.}

In chapter 4 we found that there has been a big shift in the population of users over the years, with only $45 \%-46 \%$ of all users - steady users - continuing to use the system in consecutive years. Many previous users - former users stopped using the system, and on the other hand many new users started to 
use it when the system was gradually expanded. Moreover, there is an overall decrease in frequency of system usage by all users in due time (even though the system was expanded in that period), in particular by steady users, although new users used the system more frequently than former users.

There is no significant difference in the spatial distribution pattern of both demand and demand to supply ratio (D/S) between, before and after the system expansion. The high demand areas are concentrated in the city centre and are occupied by the older stations, and the low demand areas are at the outskirts. It can be explained by the fact that the city centre area has the highest density of population, highest density of bike stations, and mixed land use patterns. However, also in high demand areas there is a decrease in use over the years, due to the reduced demand by steady users and lower demand of new users (compared to former users). It implies that stations in these high demand areas are gradually perceived as less attractive which might be caused by the fact that the novelty has disappeared or a reduced performance of the system (e.g. quality of the bikes, unavailability of bikes or parking slots).

In some areas (within $3 \mathrm{~km}$ buffer of city government) with stations from before and after system expansion, less demand by both new users and steady users was observed at the older stations after expansion. Moreover, the spatial distribution of $D / S$ reveals that these areas showed a high $D / S$ before expansion, but that this value decreased after building a new station. It suggests that again nearby stations compete with each other, and that building new stations in former high D/S areas can ease the access to the system in those areas. In addition, differences in demand over the urban area between weekdays and weekends reveals that probably users mainly cycle for commuting on weekdays, and for shopping and recreation in weekends.

In general, expanding the original system not only enabled the original users to reach new areas but also attracted new users. Adding new stations in the areas where demand or density of stations is high can both attract new users as well as serve original users. On the other hand, adding new stations in areas with lower station density further away from the city centre is mainly useful for attracting new users.

For further expansion of bike-sharing systems, it is suggested that it would be better to first investigate spatial patterns of users' demands and system's supply to uncover high and low level of demand as well as the ratio of demand to supply across the urban area. Next, it is suggested to build new stations in an area that has an excessive ratio of demand to supply rather than to expand the system to new areas unless there is a clear necessity for serving new areas. Building new stations in areas with high ratio of demand to supply not only 
extends the service area of the system but also mitigates the difficulty of finding a public bike or a parking slot.

\subsubsection{Objective 4: To examine the travel behaviour of bike- sharing users.}

In chapter 5, to shed light on the characteristics of individual trips, trip chains, and transition activities, individual trips have been classified into loop trips and directional trips, and classified trip chains into loop and non-loop trip chains. In addition, the transition activities are classified into two categories: transfer at the same station and transfer at different stations. The statistics indicate that only $4.8 \%$ of weekday trips are loop trips that start and end at the same station, which might be associated with recreational activities (physical exercises), or short-time activities (e.g. quick shopping) (Bordagaray et al., 2016). $51 \%$ of trip chains belong to loop trip chains that might be related to commuting activities. Although $49 \%$ of trip chains are non-loop trip chains, most of those trip chains show that the network distance between the origin of the first trip and the destination of the final trip is less than $600 \mathrm{~m}$. It suggests that many non-loop trip chains are potential loop trip chains, for which the capacity of the origin station is full so that users have to choose a nearby station to return the bike for the final trip. In regard to transition activities, $66 \%$ of transition activities show transfer at the same station. These findings also suggest that transferring to another nearby station is needed to get a bike because the first choice station is empty. It also suggests that neighbouring stations are complementary to each other.

Findings from the Origin-Destination matrices of single trips indicate three dominant patterns: morning peak hours (trips both start and end at 7-9 a.m.), evening peak hours (trips both start and end at 5-7 p.m.), and lunch peak hours (trips both start and end at 12-1p.m.). The vast majority of trips occur between stations nearing residential, commercial, and institutional areas, due to the site-selection of stations in the bike-sharing system. Findings from the O-D matrices of trip chains show another predominant pattern: the first trip starts at 7-8 or 8-9 a.m., and the final trip ends at 5-6 or 6-7 p.m. It accounts for the largest amount of trip chains, and suggests that the majority of these trip chains relate to commuting. According to the transition matrices, three primary patterns are identified: (1) the interval time is around 10-11 hours (i.e. arrive at 7-9 a.m. and start again at 5-7 p.m.); (2) the interval time is around 3-4 hours (i.e. arrive at 7-9 a.m. and start again at 12 p.m.); and (3) the interval time is $1-2$ hours (i.e. arrive at 12 p.m. and start again at 2 p.m.). These findings suggest that people used public bikes for commuting mainly, while some of them went home during lunch break. Some users show an afterwork shopping activity. 
Insights gained from this study have several policy implications. Firstly, the planning and role of bike-sharing systems (e.g. stand-alone use or feeder mode, inner or outer areas) should be set in the context of urban features, in terms of the urban size, housing-job ratio, and local modal split. Secondly, neighbouring stations have a complementary relationship with each other, especially when stations' capacity cannot satisfy users' demand. Rational decisions on the spacing and density of stations are important for the efficient usage of systems. Thirdly, the synergy of bike-sharing systems with other public transports modes depends on the level-of-service and use of these public transport modes as well, and thereby the attractiveness to make a multimodal trip of bike-sharing with other public transport.

\subsection{Reflections}

1. In contrast to western cities for which different activity patterns can be observed during weekdays and weekends, Zhongshan's system shows a weekend pattern that is very similar in shape to the weekday pattern with the same travel demand peaks, which can be attributed to the fact that many people in China commute both on weekdays and in weekends (O'Brien et al., 2014; Zhao et al., 2015). Moreover, Zhongshan's system shows a lunch time pattern, which might be attributed to a common Chinese lifestyle that users use the system to go home for lunch and then use it again to return to work (O'Brien et al., 2014). In contrast with bikesharing trip duration of Western cities (e.g. Melbourne, Brisbane, Washington D.C.) that fall within a tight band of between 16 and 22 mins (Fishman, 2016), Chinese systems show a relatively wide range of trip duration (e.g. 10 mins $-1 \mathrm{~h}$ ) although the average trip duration is quite short (e.g. 16 mins of weekday trips in Zhongshan). This can be explained by two reasons: firstly, Chinese systems are lower compact than European systems and tend to cover separate and distinct communities in a large city, so that Chinese systems serve trips not only occurred across communities but also occurred in a local community (O'Brien et al., 2014); and secondly, the free time for bike-sharing use is one hour in most Chinese cities but 30 mins in western cities (Fishman, 2016; Zhao et al., 2014).

2. Zhongshan's bike-sharing system mainly serves as a single mode for users to complete entire trips, rather than an intuitive feeder mode to the existing public transport system. This is similar to the BSS use in Zhuzhou city, a medium-sized Chinese city (Zhang et al., 2015). However, in large cities, such as Beijing, Hangzhou, with well-developed public transport systems, public bikes acted as both a supplement and a competitor to existing public transport systems (Campbell et al., 2016; Shaheen et al., 2011). This implies that the urban size, local model split and the level-of- 
service of existing public transport in a city affect the role of its bikesharing system.

3. It seems that Zhongshan's bike-sharing system does not serve the 'last mile' of PT. This can mainly be attributed to a low modal split and a less attractive public bus system. To change such situation, on the one hand the public bus infrastructure and accessibility need to be improved, like increasing the number of bus stops and bus lines to increase the coverage of the whole urban area. On the other hand, for commuting purpose bike stations, the site-selection of these stations should be linked to locations of bus stops, in order to reduce the transfer time.

4. In our study, the operational database consists of the following items for each trip made: user ID, bike ID, pickup and return station, and start and end time of the trip, while user ID has been coded for privacy reasons. However, the data, like socio-economic characteristics of its users and home locations of users, cannot be extracted directly from the operational database. Such data can provide information on access/egress distances and the choice for starting a trip from a bike station in the morning, which can be relevant for planning purposes.

5. Based on items included in the operational database, the information on users' behaviour and system usage, like bike-sharing demand, the most suitable locations for bike-stations, the necessary capacity of a bikestations, or travel patterns can be extracted from the operational database. For the large volume of database, Python and data mining techniques (e.g. cluster analysis) can be used to process the information and analyse the activity patterns. Moreover, performing spatial statistics analyses allow researchers to identify geographic patterns, the locations of statistically significant hot and cold spots as well as spatial outliers, or model spatial relationships.

\subsection{Recommendations for future research}

With the development of a bike-sharing system, to improve the system and to make it more sustainable rather than a short-lived project, this research is aligned with a tendency for operators and researchers to investigate the system usage and travel behaviour of bike-sharing users based on the trip data that discloses more information than the station-based data. That was also the motivation to conduct this study. Although bike-sharing is booming, the bikesharing systems and relevant studies are still developing, especially in China, and therefore several problems were encountered in conducting this research. Some of these were solved during the course of this research, and some still remain to be explored by further research. As a conclusion, we propose several areas for further research. 
1. Longer monitoring of the use of the system, travel patterns and dynamics. It is necessary to collect and analyse the trip data over the long term, in order to investigate why a large group of people stop using the system in due time and whether stopping is related to the quality of service of the bike-sharing system or other issues like the not well developed bike infrastructure.

2. Analyse characteristics of the trip makers, if socio-economic information can be linked to the user ID. Of course there might be privacy issues, but this information could be very valuable to analyse trip patterns in combination with socio-economic characteristics. In addition, knowing the residential location of the user ID could be useful to analyse the access characteristics to the BSS, but here privacy might be even more an issue. To deal with such problem, the data owners can pre-process the original data, like using a proxy that can protect the users' privacy to represent a user's residential/job location.

3. Finding a proper balance between station density and capacity (number of parking slots), as well as its relation with neighbourhood characteristics can be beneficial for planning location-allocations of bike-sharing stations to improve the turnover at bike station

4. Further expanding and improving the bicycle infrastructure or network might have a positive influence on the use of the bike-sharing system. If some improvements are considered and planned, a thorough spatial analysis could be conducted to analyse its impacts on BSS use and travel patterns.

5. Analyse the trip data at bicycle level. As the operational database contains the data of bike ID, by monitoring the performance of each bike maintenance issues can become apparent and renewal programs could be set up.

6. Investigate by user survey. User survey can be conducted to investigate two aspects. Firstly, the short distance of bike trips and the absence of a clear orientation might be a consequence of a lack of or not overall well developed bike infrastructure; secondly, parts of users' travel behaviour, like the potential travel purpose of bike-sharing trips, cannot be explicitly explained by the information embedded in the operational database.

7. Developing a geo-spatial assessment method for location-allocation of bike-sharing systems in collaborative decision support environment: the guideline on building bike-sharing systems is quite the same all over the world at the present stage, which does not employ geo-spatial assessment 
techniques for determining the location-allocation of bike stations. However, the location-allocation of bike stations affects the usage of bike stations. Moreover, the spatial characteristics of urban environment also have impact on the usage of public bikes at stations. Furthermore, neighbouring bike stations also have correlation with each other. To support a better planning and performance of bike sharing systems, it is necessary to develop a geo-spatial assessment method for strategic planning of bike sharing system, incorporating the opinions of stakeholders.

8. Problems of station rebalancing: redistribution of public bikes has been common issue shown in the implementation of bike-sharing systems. As we known, bike stations are equipped with limited number of parking slots and public bikes. Some of the stations have high demands for picking up or dropping off public bikes during peak hours, but others not. It is therefore necessary to design a framework to identify the variation of station capacity during the course of day and address how to redistribute public bikes between stations, in order to satisfy users' demands for renting and returning public bikes as well as enhance the efficiency of system usage. 


\section{Bibliography}

Ahillen, M., Mateo-Babiano, D., Corcoran, J. (2016). Dynamics of bike sharing in Washington, DC and Brisbane, Australia: Implications for policy and planning. International Journal of Sustainable Transportation, 10(5), 441454. doi:10.1080/15568318.2014.966933

Anselin, L., Rey, S. J. (2014). Modern Spatial Econometrics in Practice: A Guide to GeoDa, GeoDaSpace and PySAL. Chicago: GeoDa Press.

Bachand-Marleau, J., Lee, B., El-Geneidy, A. (2012). Better understanding of factors influencing likelihood of using shared bicycle systems and frequency of use. Transportation Research Record, 2314, 66-71. doi:http://dx.doi.org/10.3141/2314-09

Barter, P., Kenworthy, J., Laube, F. (2003). Lessons from Asia on Sustainable Urban Transport. In N. Low \& B. Gleeson (Eds.), Making Urban Transport Sustainable (pp. 252-270): Palgrave Macmillan.

Beecham, R., Wood, J. (2014). Exploring gendered cycling behaviours within a large-scale behavioural data-set. Transportation Planning and Technology, 37(1), 83-97. doi:10.1080/03081060.2013.844903

Bordagaray, M., Dell'Olio, L., Fonzone, A., Ibeas, A. (2016). Capturing the conditions that introduce systematic variation in bike-sharing travel behaviour using data mining techniques. Transportation Research Part CEmerging Technologies, 71, 231-248. doi:10.1016/j.trc.2016.07.009

Borgnat, P., Abry, P., Flandrin, P., Robardet, C., Rouquier, J. B., Fleury, E. (2011). SHARED BICYCLES IN A CITY: A SIGNAL PROCESSING AND DATA ANALYSIS PERSPECTIVE. Advances in Complex Systems, 14(3), 415-438. doi:10.1142/s0219525911002950

Buck, D., Buehler, R. (2012). Bike Lanes and Other Determinants of Capital Bikeshare Trips. Paper presented at the Transportation Research Board 91st Annual Meeting, Washington DC. https://trid.trb.org/view.aspx?id=1130348

Bührmann, S. (2008). Bicycles ad public-individual transport-European Developments. Paper presented at the Meetbike Conference, Dresden. http://www.gtkp.com/uploads/20091127-150125-6592Meetbike article Buehrmann 040408.pdf

Bullock, C., Brereton, F., Bailey, S. (2017). The economic contribution of public bike-share to the sustainability and efficient functioning of cities. Sustainable Cities and Society, 28, 76-87. doi:http://dx.doi.org/10.1016/j.scs.2016.08.024

Campbell, A. A., Cherry, C. R., Ryerson, M. S., Yang, X. (2016). Factors influencing the choice of shared bicycles and shared electric bikes in Beijing. Transportation Research Part C: Emerging Technologies, 67, 399414. doi:http://dx.doi.org/10.1016/j.trc.2016.03.004

Castillo-Manzano, J. I., Castro-Nuño, M., López-Valpuesta, L. (2015). Analyzing the transition from a public bicycle system to bicycle ownership: 
A complex relationship. Transportation Research Part D: Transport and Environment, 38, 15-26. doi:http://dx.doi.org/10.1016/j.trd.2015.04.004

Cervero, R., Kockelman, K. (1997). Travel demand and the 3Ds: Density, diversity, and design. Transportation Research Part D: Transport and Environment, 2(3), 199-219. doi:http://dx.doi.org/10.1016/S13619209(97)00009-6

Cervero, R., Sarmiento, O. L., Jacoby, E., Gomez, L. F., Neiman, A. (2009). Influences of Built Environments on Walking and Cycling: Lessons from Bogota. International Journal of Sustainable Transportation, 3(4), 203226. doi: $10.1080 / 15568310802178314$

Corcoran, J., Li, T. (2014). Spatial analytical approaches in public bicycle sharing programs. Journal of Transport Geography, 41, 268-271. doi:http://dx.doi.org/10.1016/j.jtrangeo.2014.09.005

Corcoran, J., Li, T., Rohde, D., Charles-Edwards, E., Mateo-Babiano, D. (2014). Spatio-temporal patterns of a Public Bicycle Sharing Program: the effect of weather and calendar events. Journal of Transport Geography, 41, 292305. doi: http://dx.doi.org/10.1016/j.jtrangeo.2014.09.003

CSD. (2011). BICYCLE-SHARING SCHEMES:ENHANCING SUSTAINABLE MOBILITY IN URBAN AREAS (CSD19/2011/BP8). Retrieved from New York:

http://www.un.org/esa/dsd/susdevtopics/sdt tran documents.shtml

da Silva, A. N. R., da Silva Costa, M., Macedo, M. H. (2008). Multiple views of sustainable urban mobility: The case of Brazil. Transport Policy, 15(6), 350-360. doi:10.1016/j.tranpol.2008.12.003

Daddio, D. W. (2012). Maximizing bicycle sharing: an empirical analysis of capital bikeshare usage. (Master Master Thesis), University of North Carolina at Chapel Hill. Retrieved from http://rethinkcollegepark.net/blog/wpcontent/uploads/2006/07/DaddioMP Final-Draft.pdf

de Vasconcellos, E. A. (2005). Urban change, mobility and transport in São Paulo: three decades, three cities. Transport Policy, 12(2), 91-104. doi: 10.1016/j.tranpol.2004.12.001

Dell'Amico, M., Iori, M., Stefano, N., Stützle, T. (2016). A Destroy and Repair Algorithm for the Bike sharing Rebalancing Problem. Computers \& Operations Research. doi:http://dx.doi.org/10.1016/j.cor.2016.01.011

dell'Olio, L., Ibeas, A., Moura, J. L. (2011). Implementing bike-sharing systems. Proceedings of the Institution of Civil Engineers-Municipal Engineer, 164(2), 89-101. doi:10.1680/muen.2011.164.2.89

DeMaio, P. (2008, 7th October). The Bike-sharing Phenomenon - The History of Bike-sharing. Carbusters Magazine.

DeMaio, P. (2009). Bike-sharing:History, Impacts, Models of Provision, and Future. Public Transportation, 12, 16. 
El-Assi, W., Salah Mahmoud, M., Nurul Habib, K. (2015). Effects of built environment and weather on bike sharing demand: a station level analysis of commercial bike sharing in Toronto. Transportation, 1-25. doi:10.1007/s11116-015-9669-z

EuropeanCommission. (2007). Towards a new culture for urban mobility. Retrieved from http://ec.europa.eu/transport/urban/urban_mobility/green_paper/doc/2 $007 \quad 0925$ gp urban mobility memo en.pdf

Ewing, R., Cervero, R. (2001). Travel and the built environment: A synthesis. Transport Research Record(1780), 87-114. doi: $\underline{\text { http://dx.doi.org/10.3141/1780-10 }}$

Ewing, R., Cervero, R. (2010). Travel and the Built Environment. Journal of the American Planning Association, 76(3), 265-294. doi:10.1080/01944361003766766

Faghih-Imani, A., Eluru, N. (2015). Analysing bicycle-sharing system user destination choice preferences: Chicago's Divvy system. Journal of Transport Geography, 44, 53-64. doi:http://dx.doi.org/10.1016/j.jtrangeo.2015.03.005

Faghih-Imani, A., Eluru, N. (2016). Incorporating the impact of spatiotemporal interactions on bicycle sharing system demand: A case study of New York CitiBike system. Journal of Transport Geography, 54, 218-227. doi:http://dx.doi.org/10.1016/j.jtrangeo.2016.06.008

Faghih-Imani, A., Eluru, N., El-Geneidy, A. M., Rabbat, M., Haq, U. (2014). How land-use and urban form impact bicycle flows: evidence from the bicycle-sharing system (BIXI) in Montreal. Journal of Transport Geography, 41, 306-314. doi:http://dx.doi.org/10.1016/j.jtrangeo.2014.01.013

Field, A. (2009). Discovering Statistics Using SPSS. London: SAGE Publications Ltd.

Fishman, E. (2016). Bikeshare: A Review of Recent Literature. Transport Reviews, 36(1), 92-113. doi:10.1080/01441647.2015.1033036

Fishman, E., Washington, S., Haworth, N. (2013). Bike Share: A Synthesis of the Literature. Transport Reviews, 33(2), 148-165. doi:10.1080/01441647.2013.775612

Fishman, E., Washington, S., Haworth, N. (2014). Bike share's impact on car use: Evidence from the United States, Great Britain, and Australia. Transportation Research Part D: Transport and Environment, 31, 13-20. doi:http://dx.doi.org/10.1016/j.trd.2014.05.013

Fishman, E., Washington, S., Haworth, N., Mazzei, A. (2014). Barriers to bikesharing: an analysis from Melbourne and Brisbane. Journal of Transport Geography, 41, 325-337. doi:http://dx.doi.org/10.1016/j.jtrangeo.2014.08.005 
Frade, I., Ribeiro, A. (2014). Bicycle Sharing Systems Demand. Procedia Social and Behavioral Sciences, 111, 518-527. doi:http://dx.doi.org/10.1016/j.sbspro.2014.01.085

Froehlich, J., Neumann, J., Oliver, N. (2009). Sensing and Predicting the Pulse of the City through Shared Bicycling. Paper presented at the 21st International Joint Conference on Artificial intelligence, Pasadena, California, USA.

Fuller, D., Gauvin, L., Kestens, Y., Daniel, M., Fournier, M., Morency, P., Drouin, L. (2011). Use of a New Public Bicycle Share Program in Montreal, Canada. American Journal of Preventive Medicine, 41(1), 80-83. doi:10.1016/j.amepre.2011.03.002

Fuller, D., Sahlqvist, S., Cummins, S., Ogilvie, D. (2012). The impact of public transportation strikes on use of a bicycle share program in London: Interrupted time series design. Preventive Medicine, 54(1), 74-76. doi:10.1016/j.ypmed.2011.09.021

Gakenheimer, R. (1999). Urban mobility in the developing world. Transportation Research Part A: Policy and Practice, 33(7-8), 671-689. doi:10.1016/s0965-8564(99)00005-1

García-Palomares, J. C., Gutiérrez, J., Latorre, M. (2012). Optimizing the location of stations in bike-sharing programs: A GIS approach. Applied Geography, 35(1-2), 235-246. doi:10.1016/j.apgeog.2012.07.002

Gauthier, A., Hughes, C., Kost, C., Li, S., Linke, C., Lotshaw, S., . . Treviño, X. (2013). ITDP Bike-Share Planning Guide. Retrieved from https://www.itdp.org/wpcontent/uploads/2014/07/ITDP Bike Share Planning Guide.pdf

Gebhart, K., Noland, R. B. (2014). The impact of weather conditions on bikeshare trips in Washington, DC. Transportation, 41(6), 1205-1225. doi:10.1007/s11116-014-9540-7

Gonzalez, F., Melo-Riquelme, C., de Grange, L. (2016). A combined destination and route choice model for a bicycle sharing system. Transportation, 43(3), 407-423. doi:10.1007/s11116-015-9581-6

Goodman, A., Cheshire, J. (2014). Inequalities in the London bicycle sharing system revisited: impacts of extending the scheme to poorer areas but then doubling prices. Journal of Transport Geography, 41. doi:http://dx.doi.org/10.1016/j.jtrangeo.2014.04.004

Grotenhuis, J.-W., Wiegmans, B. W., Rietveld, P. (2007). The desired quality of integrated multimodal travel information in public transport: Customer needs for time and effort savings. Transport Policy, 14(1), 27-38.

Gutiérrez, J., Cardozo, O. D., García-Palomares, J. C. (2011). Transit ridership forecasting at station level: an approach based on distance-decay weighted regression. Journal of Transport Geography, 19(6), 1081-1092. doi:http://dx.doi.org/10.1016/j.jtrangeo.2011.05.004 
Han, S. S. (2010). Managing motorization in sustainable transport planning: the Singapore experience. Journal of Transport Geography, 18(2), 314321. doi:10.1016/j.jtrangeo.2009.06.010

Heesch, K. C., Giles-Corti, B., Turrell, G. (2015). Cycling for transport and recreation: Associations with the socio-economic, natural and built environment. Health \& Place, 36, 152-161. doi:10.1016/j.healthplace.2015.10.004

Hine, J., Scott, J. (2000). Seamless, accessible travel: Users' views of the public transport journey and interchange. Transport Policy, 7(3), 217-226.

ITDP-China. (2015). China Bikesharing. Retrieved from http://www.publicbike.net/defaulten.aspx

Jäppinen, S., Toivonen, T., Salonen, M. (2013). Modelling the potential effect of shared bicycles on public transport travel times in Greater Helsinki: An open data approach. Applied Geography, 43, 13-24. doi:http://dx.doi.org/10.1016/j.apgeog.2013.05.010

Jensen, P., Rouquier, J.-B., Ovtracht, N., Robardet, C. (2010). Characterizing the speed and paths of shared bicycle use in Lyon. Transportation Research Part D: Transport and Environment, 15(8), 522-524. doi:10.1016/j.trd.2010.07.002

Jiménez, P., Nogal, M., Caulfield, B., Pilla, F. (2016). Perceptually important points of mobility patterns to characterise bike sharing systems: The Dublin case. Journal of Transport Geography, 54, 228-239. doi:http://dx.doi.org/10.1016/j.jtrangeo.2016.06.010

Kadri, A., Kacem, I., Labadi, K. (2016). A branch-and-bound algorithm for solving the static rebalancing problem in bicycle-sharing systems. Computers \& Industrial Engineering. doi:http://dx.doi.org/10.1016/j.cie.2016.02.002

Kager, R., Bertolini, L., Te Brommelstroet, M. (2016). Characterisation of and reflections on the synergy of bicycles and public transport. Transportation Research Part a-Policy and Practice, 85, 208-219. doi:10.1016/j.tra.2016.01.015

Kaltenbrunner, A., Meza, R., Grivolla, J., Codina, J., Banchs, R. (2010). Urban cycles and mobility patterns: Exploring and predicting trends in a bicyclebased public transport system. Pervasive and Mobile Computing, 6(4), 455-466. doi:10.1016/j.pmcj.2010.07.002

Keijer, M. J. N., Rietveld , P. (2000). How do people get to the railway station? The Dutch experience. Transportation Planning and Technology, 23(3), 215-235. doi:10.1080/03081060008717650

Kemperman, A., Timmermans, H. (2009). Influences of Built Environment on Walking and Cycling by Latent Segments of Aging Population. Transportation Research Record(2134), 1-9. doi:10.3141/2134-01

Krizek, K. J., Barnes, G., Thompson, K. (2009). Analyzing the Effect of Bicycle Facilities on Commute Mode Share over Time. Journal of Urban Planning 
and Development-Asce, 135(2), 66-73. doi:10.1061/(asce)07339488(2009)135:2(66)

Krizek, K. J., Johnson, P. J. (2006). Proximity to trails and retail: Effects on urban cycling and walking. Journal of the American Planning Association, 72(1), 33-42. doi:10.1080/01944360608976722

Lathia, N., Ahmed, S., Capra, L. (2012). Measuring the impact of opening the London shared bicycle scheme to casual users. Transportation Research Part C: Emerging Technologies, 22, 88-102. doi:10.1016/j.trc.2011.12.004

Lin, J.-R., Yang, T.-H. (2011). Strategic design of public bicycle sharing systems with service level constraints. Transportation Research Part E: Logistics and Transportation Review, 47(2), 284-294. doi:10.1016/j.tre.2010.09.004

Liu, Z., Jia, X., Cheng, W. (2012). Solving the Last Mile Problem: Ensure the Success of Public Bicycle System in Beijing. Procedia - Social and Behavioral Sciences, 43, 73-78. doi:10.1016/j.sbspro.2012.04.079

Martens, K. (2007). Promoting bike-and-ride: The Dutch experience. Transportation Research Part a-Policy and Practice, 41(4), 326-338. doi:10.1016/j.tra.2006.09.010

Martin, E. W., Shaheen, S. A. (2014). Evaluating public transit modal shift dynamics in response to bikesharing: a tale of two U.S. cities. Journal of Transport Geography, 41, 315-324. doi:http://dx.doi.org/10.1016/j.jtrangeo.2014.06.026

Massink, R., Zuidgeest, M. H. P., Rijnsburger, J., Sarmiento, O. L., van Maarseveen, M. F. A. M. (2011). The climate value of cycling. Natural Resources Forum, 35(2), 100-111.

Mateo-Babiano, I., Bean, R., Corcoran, J., Pojani, D. (2016). How does our natural and built environment affect the use of bicycle sharing? Transportation Research Part A: Policy and Practice, 94, 295-307. doi:http://dx.doi.org/10.1016/j.tra.2016.09.015

Maurer, L. K. (2012). Feasibility Study for a Bicycle Sharing Program in Sacramento, California. Paper presented at the Transportation Research Board 91st Annual Meeting, Washington DC. https://trid.trb.org/view.aspx?id=1130876

Meddin, R., DeMaio, P. (2016). The Bike-sharing World Map. Retrieved from www.bikesharingmap.com

Midgley, P. (2009). The Role of Smart Bike Sharing Systems in Urban Mobility. Journeys(2), 23-31.

MOHURD. (2012). Code for Design of Urban Road Engineering. China: Ministry of Housing and Urban-Rural Development.

Moudon, A. V., Lee, C., Cheadle, A. D., Collier, C. W., Johnson, D., Schmid, T. L., Weather, R. D. (2005). Cycling and the built environment, a US perspective. Transportation Research Part D: Transport and Environment, 10(3), 245-261. doi:http://dx.doi.org/10.1016/j.trd.2005.04.001 
Murphy, E., Usher, J. (2015). The Role of Bicycle-sharing in the City: Analysis of the Irish Experience. International Journal of Sustainable Transportation, 9(2), 116-125. doi:10.1080/15568318.2012.748855

Nair, R., Miller-Hooks, E., Hampshire, R. C., Bušić, A. (2013). Large-Scale Vehicle Sharing Systems: Analysis of Vélib'. International Journal of Sustainable Transportation, 7(1), 85-106. doi: $10.1080 / 15568318.2012 .660115$

Neumann-Saavedra, B. A., Vogel, P., Mattfeld, D. C. (2015). Anticipatory Service Network Design of Bike Sharing Systems. Transportation Research Procedia, 10 , 355-363. doi:http://dx.doi.org/10.1016/j.trpro.2015.09.085

Nikitas, A., Wallgren, P., Rexfelt, O. (2016). The paradox of public acceptance of bike sharing in Gothenburg. Proceedings of the Institution of Civil Engineers-Engineering Sustainability, 169(3), 101-113. doi: $10.1680 /$ jensu. 14.00070

Nkurunziza, A., Zuidgeest, M., Brussel, M., Van Maarseveen, M. (2012). Examining the potential for modal change: Motivators and barriers for bicycle commuting in Dar-es-Salaam. Transport Policy, 24, 249-259. doi:https://doi.org/10.1016/j.tranpol.2012.09.002

O'Neil, P., Caulfield, B. (2012). Examining user behaviour on a shared bike scheme: the case of Dublin Bikes. Paper presented at the The 13th International Conference on Travel Behaviour Research, Toronto. http://hdl.handle.net/2262/68119

O'Brien, O., Cheshire, J., Batty, M. (2014). Mining bicycle sharing data for generating insights into sustainable transport systems. Journal of Transport Geography, 34, 262-273. doi:http://dx.doi.org/10.1016/j.jtrangeo.2013.06.007

Oliveira, G. N., Sotomayor, J. L., Torchelsen, R. P., Silva, C. T., Comba, J. L. D. (2016). Visual analysis of bike-sharing systems. Computers \& Graphics, 60, 119-129. doi: http://dx.doi.org/10.1016/j.cag.2016.08.005

Parkes, S. D., Marsden, G., Shaheen, S. A., Cohen, A. P. (2013). Understanding the diffusion of public bikesharing systems: evidence from Europe and North America. Journal of Transport Geography, 31, 94-103. doi:http://dx.doi.org/10.1016/j.jtrangeo.2013.06.003

Pucher, J., Dill, J., Handy, S. (2010). Infrastructure, programs, and policies to increase bicycling: An international review. Preventive Medicine, 50, S106-S125. doi:10.1016/j.ypmed.2009.07.028

Ricci, M. (2015). Bike sharing: A review of evidence on impacts and processes of implementation and operation. Research in Transportation Business \& Management, 15 , 28-38. doi:http://dx.doi.org/10.1016/j.rtbm.2015.03.003

Richardson, B. C. (2005). Sustainable transport: analysis frameworks. Journal of Transport Geography, 13(1), 29-39. doi:10.1016/j.jtrangeo.2004.11.005 
Rietveld, P., Daniel, V. (2004). Determinants of bicycle use: do municipal policies matter? Transportation Research Part A: Policy and Practice, 38(7), 531-550. doi:10.1016/j.tra.2004.05.003

Rixey, R. (2013). Station-level forecasting of bikesharing ridership: Station Network Effects in Three U.S. Systems. Transportation Research Record(2387), 46-55. doi:10.3141/2387-06

Rudloff, C., Lackner, B. (2014). Modeling Demand for Bikesharing Systems Neighboring Stations as Source for Demand and Reason for Structural Breaks. Transportation Research Record(2430), 1-11. doi:10.3141/243001

Shaheen, S., Guzman, S., Zhang, H. (2010). Bikesharing in Europe, the Americas, and Asia Past, Present, and Future. Transportation Research Record(2143), 159-167. doi:10.3141/2143-20

Shaheen, S., Martin, E., Cohen, A. P., Finson, R. S. (2012). Public Bikesharing in North America: Early Operator and User Understanding. Retrieved from San José State University, California:

Shaheen, S., Zhang, H., Martin, E., Guzman, S. (2011). Hangzhou Public Bicycle: Understanding Early Adoption and Behavioral Response to Bikesharing in Hangzhou, China. Transportation Research Record(2247), 34-41. doi: http://dx.doi.org/10.3141/2247-05

Sudhakara Reddy, B., Balachandra, P. (2012). Urban mobility: A comparative analysis of megacities of India. Transport Policy, 21(0), 152-164. doi: 10.1016/j.tranpol.2012.02.002

Tang, Y., Pan, H., Shen, Q. (2011). Bike-Sharing Systems in Beijing, Shanghai, and Hangzhou and Their Impact on Travel Behavior. Paper presented at the Transportation Research Board 90th Annual Meeting, Washington DC. http://trid.trb.org/view.aspx?id=1093278

TransportCanada. (2009). BIKE-SAHRING GUIDE. (T22-180/2009E). Canada: Minister of Transport Retrieved from http://transact-en.tc.gc.ca.

Van Acker, V., Derudder, B., Witlox, F. (2013). Why people use their cars while the built environment imposes cycling. Journal of Transport and Land Use, 6(1), 53-62. doi: 10.5198/jtlu.v6i1.288

Vandenbulcke, G., Dujardin, C., Thomas, I., de Geus, B., Degraeuwe, B., Meeusen, R., Panis, L. I. (2011). Cycle commuting in Belgium: Spatial determinants and 're-cycling' strategies. Transportation Research Part aPolicy and Practice, 45(2), 118-137. doi:10.1016/j.tra.2010.11.004

Vogel, M., Hamon, R., Lozenguez, G., Merchez, L., Abry, P., Barnier, J., . . . Robardet, C. (2014). From bicycle sharing system movements to users: a typology of Vélo'v cyclists in Lyon based on large-scale behavioural dataset. Journal of Transport Geography, 41, 280-291. doi:http://dx.doi.org/10.1016/j.jtrangeo.2014.07.005

Vogel, P., Greiser, T., Mattfeld, D. C. (2011). Understanding Bike-Sharing Systems using Data Mining: Exploring Activity Patterns. Procedia - Social and Behavioral Sciences, 20, 514-523. doi:10.1016/j.sbspro.2011.08.058 
Wang, X., Lindsey, G., Schoner, J. E., Harrison, A. (2016). Modeling Bike Share Station Activity: Effects of Nearby Businesses and Jobs on Trips to and from Stations. Journal of Urban Planning and Development, 142(1), 04015001. doi:doi:10.1061/(ASCE)UP.1943-5444.0000273

Wardman, M., Tight, M., Page, M. (2007). Factors influencing the propensity to cycle to work. Transportation Research Part a-Policy and Practice, 41(4), 339-350. doi:10.1016/j.tra.2006.09.011

Wu, L., Zhi, Y., Sui, Z., Liu, Y. (2014). Intra-Urban Human Mobility and Activity Transition: Evidence from Social Media Check-In Data. PLoS ONE, 9(5), e97010. doi:10.1371/journal.pone.0097010

Yang, R. R., Long, R. Y. (2016). Analysis of the Influencing Factors of the Public Willingness to Participate in Public Bicycle Projects and Intervention Strategies-A Case Study of Jiangsu Province, China. Sustainability, 8(4), 16. doi:10.3390/su8040349

Zaltz Austwick, M., O'Brien, O., Strano, E., Viana, M. (2013). The Structure of Spatial Networks and Communities in Bicycle Sharing Systems. PLOS ONE, 8(9), e74685. doi:10.1371/journal.pone.0074685

Zhang, H., Shaheen, S. A., Chen, X. P. (2014). Bicycle Evolution in China: From the $1900 \mathrm{~s}$ to the Present. International Journal of Sustainable Transportation, 8(5), 317-335. doi:10.1080/15568318.2012.699999

Zhang, L., Zhang, J., Duan, Z.-y., Bryde, D. (2015). Sustainable bike-sharing systems: characteristics and commonalities across cases in urban China. Journal of Cleaner Production, 97, 124-133. doi:http://dx.doi.org/10.1016/j.jclepro.2014.04.006

Zhang, Y., Thomas, T., Brussel, M., van Maarseveen, M. (2017). Exploring the impact of built environment factors on the use of public bikes at bike stations: Case study in Zhongshan, China. Journal of Transport Geography, 58, 59-70. doi:http://dx.doi.org/10.1016/j.jtrangeo.2016.11.014

Zhao, J., Deng, W., Song, Y. (2014). Ridership and effectiveness of bikesharing: The effects of urban features and system characteristics on daily use and turnover rate of public bikes in China. Transport Policy, 35(0), 253-264. doi: http://dx.doi.org/10.1016/j.tranpol.2014.06.008

Zhao, J., Wang, J., Deng, W. (2015). Exploring bikesharing travel time and trip chain by gender and day of the week. Transportation Research Part C: Emerging Technologies, 58, Part $B, \quad$ 251-264. doi:http://dx.doi.org/10.1016/j.trc.2015.01.030

ZhongshanStatisticsBureau. (2014). Zhongshan Statistic Yearbook 2014. Retrieved from http://www.zsda.gov.cn/plus/php dq zhishu.php

Zhou, X. (2015). Understanding Spatiotemporal Patterns of Biking Behavior by Analyzing Massive Bike Sharing Data in Chicago. PLOS ONE, 10(10), e0137922. doi:10.1371/journal.pone.0137922 


\section{Summary}

Bike-sharing systems aim to increase bike use and improve accessibility of urban transit. These systems have grown in popularity and are expanding rapidly across cities worldwide. Studies have shown that cycling for both utilitarian and recreational purposes has increased in some cities after running bike-sharing programs. The latest generation of bike-sharing programs has employed information technology that produces station-based data or triplevel data. It facilitates to analyse the actual use of these systems. Researchers have shown a huge interest in studying transport supply and travel demand aspects of bike-sharing systems. Despite the interesting findings and important implications of these studies, yet a number of research gaps can be identified, like travel demand characteristics and patterns of bike-sharing systems, travel demand dynamics of bike-sharing systems, and travel behaviour of bikesharing users.

Moreover, the majority of studies dealt with American and European systems, and its findings cannot be transferred directly to Asian cites that exhibit quite different urban features and cycle demand from American and European cities. The largest amount of bike-sharing programs is running in China but only a few researches can be found in scientific literature. Within this context, this research aims to explore methods and approaches to extract information on the actual use of a bike-sharing system and the performance of the system by employing datasets selected from the operational database from the bikesharing system in the city of Zhongshan, China.

Firstly, this research unravels travel demand characteristics and patterns of system usage, in terms of system efficiency, trip characteristics, and station activity patterns. As a result, this research indicates and discusses the efficiency of the system usage based on a set of system metrics, the spatial and temporal characteristics of single trips, and the weekday and weekend patterns for bike use at the level of stations.

Secondly, this research examines the effect of surrounding built environment factors on the use of public bikes at station level. This study employs a spatial multiple linear regression model to examine and elaborate the statistical relations between surrounding built environment variables and the actual use of public bikes at stations, incorporating the spatial correlation between nearby stations. Trip demand, and the demand to supply ratio, both at bike station level, are the two dependent variables that have been estimated. The model results uncover how each built environment factors and nearby stations affect the demand as well as the D/S at station level. The findings contribute to an understanding how spatial factors drive BSS demand in the context of a 
Chinese city, to be able to plan successfully new bike-sharing systems or stations in those cities.

Thirdly, this research explores the travel demand dynamics of bike-sharing systems both from a temporal and spatial perspective, employing spatial and statistical analyses. The findings contribute to an understanding of how users and system usage change over the years, and how the expansion of the system affects the usage of the system.

Finally, this research provides a new insight into exploring the travel behaviour of bike-sharing users. Such work examines the spatial and temporal patterns of bike-sharing trips, trip chains, and transition activities. The analysis incorporates information of land use types around bike stations, time information of pickup and return actions, and transition activities in between bike trips.

This thesis contributes to a better understanding of the actual use and performance of bike-sharing systems. This research aims to develop methods and approaches to extract information from an operational database with respect to trip characteristics of bike-sharing users. It offers new research perspectives and approaches to unravel the characteristics and patterns of bike-sharing usage, like travel demand characteristics and dynamics and travel behaviour, at the level of trips, stations and users. The findings of this research can be beneficial to researchers, urban planners and policy makers to extend and improve their knowledge about such systems. This knowledge is useful for the improvement and expansion of existing systems, as well as the adoption of new systems. 


\section{Samenvatting}

Fietsdeelsystemen beogen het fietsgebruik te doen toenemen en de bereikbaarheid van het stedelijk openbaar vervoer te bevorderen. Deze systemen winnen sterk aan populariteit en verspreiden zich razendsnel over steden in de gehele wereld. Onderzoek in enkele steden heeft aangetoond dat het fietsen voor zowel recreatieve als niet-recreatieve doeleinden is toegenomen na de introductie van deelfietssystemen. De meest recente systemen maken gebruik van informatietechnologie, die het mogelijk maakt gegevens te registreren op zowel stations- als rit-niveau. Deze gegevens vereenvoudigen het uitvoeren van een analyse van het feitelijke gebruik van zo'n systeem. In toenemende mate houden wetenschappers zich bezig met onderzoek naar de vraag en aanbod aspecten van deelfietssystemen. Ondanks de interessante onderzoeksbevindingen en de daaraan verbonden gevolgtrekkingen zijn er nog steeds een aantal kennislacunes aan te geven zoals vervoersvraag karakteristieken en -patronen, de dynamiek in het gebruik van de systemen, en het vervoersgedrag van de gebruikers van deze systemen.

De meeste onderzoeken hebben betrekking op Amerikaanse en Europese systemen. De overdraagbaarheid van deze onderzoeksresultaten naar Aziatische steden is niet zonder meer gerechtvaardigd vanwege aanmerkelijke verschillen in stedelijke kenmerken en vervoersgedrag. Echter de meeste deelfietssystemen zijn operationeel in China, maar in de wetenschappelijke literatuur zijn de systemen in dit land onderbelicht. In deze context heeft dit onderzoek zich beziggehouden met het verkennen van methoden en benaderingen om informatie te extraheren over zowel het feitelijke gebruik als de performance van een deelfietssysteem uit de operationele database van een deelfietssysteem in de stad Zhongshan in China.

Ten eerste ontrafelt dit onderzoek vervoersvraag karakteristieken en patronen van systeemgebruik, in termen van systeemefficiëntie, rit karakteristieken en activiteiten patronen per station. Het onderzoek geeft indicaties voor en bespreekt de efficiëntie van het systeem op basis van een verzameling systeemgrootheden, de ruimtelijke en temporele karakteristieken van enkelvoudige ritten, en de werkdag en weekend karakteristieken van fietsgebruik per station.

Ten tweede onderzoekt deze studie de effecten van factoren van de gebouwde omgeving op het gebruik van deelfietsen per station. Het onderzoek gebruikt een ruimtelijk meervoudig lineair regressiemodel om statistische relaties te bepalen tussen gebouwde omgevingsfactoren en het feitelijke deelfiets gebruik per station, met inachtneming van ruimtelijke correlaties tussen nabijgelegen stations. De vervoersvraag in aantal ritten als ook de vraag-aanbod ratio, beide 
op het niveau van fietsstation, zijn de twee afhankelijke variabelen die zijn geschat. De modelresultaten geven inzicht in hoe elk van de gebouwde omgevingsvariabelen invloed hebben op de vervoersvraag en de vraag-aanbod ratio per station. De resultaten dragen bij aan een beter begrip hoe ruimtelijke factoren de vraag naar deelfietsen in een Chinese stad beïnvloeden, welke informatie belangrijk is bij het opzetten van nieuwe, dan wel het uitbreiden van bestaande deelfietssystemen.

Ten derde richt deze studie zich op de dynamiek in de vervoersvraag van het deelfietssysteem zowel in ruimtelijk als temporeel perspectief, waarbij gebruik wordt gemaakt van ruimtelijke en statistische analyses. De resultaten vergroten de kennis over het verloop van systeemgebruikers en -gebruik over de jaren, en over de invloed van uitbreiding van het systeem op het gebruik.

Tenslotte verschaft dit onderzoek nieuwe inzichten in hoe vervoersgedrag van deelfiets gebruikers kan worden verkend. In het bijzonder betreft het ruimtelijke en temporele kenmerken van deelfiets ritten, ritketens en transitie activiteiten. De analyse benut informatie over landgebruik typen in de directe omgeving van fietsstations, tijdstippen van ophaal en retour acties van deelfietsen, als ook van transitie activiteiten tussen opvolgende fietsritten.

Dit onderzoek draagt bij aan een beter begrip van het feitelijke gebruik en de performance van deelfietssystemen. De studie beoogt methoden en benaderingen te ontwikkelen om met gebruikmaking van de operationele database informatie in te winnen met betrekking tot ritkarakteristieken van deelfiets gebruikers. Het biedt nieuwe onderzoeksperspectieven en benaderingen om karakteristieken en patronen van deelfiets gebruik te analyseren, zoals vervoersvraag karakteristieken, de dynamiek in gebruik, als ook vervoersgedrag vanuit de invalshoek van ritten, stations en gebruikers. De resultaten van dit onderzoek zijn van belang voor onderzoekers, stedelijke planners en beleidsmakers vanwege het verdiepen en verbeteren van de kennis over het functioneren van dergelijke systemen. Deze kennis is met name van belang bij het opzetten van nieuwe, of het uitbreiden van bestaande deelfietssystemen. 


\section{Biography}

Ying Zhang was born in Xinyang, China, on 13 July 1987. She received her MSc degrees in urban planning and Geo-information management in 2011 under a joint master's program between Wuhan University and Faculty of ITC of the University of Twente. In March 2012, she began her PhD project at PGM department of the Faculty of ITC of the University of Twente, which was supported by a four-year doctoral scholarship from the China Scholarship Council and the research fund of ITC. Her PhD research aims at investigating the bike-sharing usage by mining on the trip data of bike-sharing users. During her PhD study, she has published journal papers, and attended international conferences and various courses. Her PhD research has resulted in the following publications.

Peer-reviewed journal articles:

Zhang, Y., Brussel, M.J.G., Thomas, T., van Maarseveen, M.F.A.M. (2017). Mining bike-sharing travel behaviour data: an investigation into trip chains and transition activities. Journal of Computers, Environment and Urban Systems, (under review).

Zhang, Y., Thomas, T., Brussel, M.J.G. and van Maarseveen, M.F.A.M. (2017) Exploring the impact of built environment factors on the use of public bikes at bike stations: case study in Zhongshan, China. In: Journal of transport geography, 58 (2017) pp. 59-70.

Zhang, Y., Thomas, T., Brussel, M.J.G. and van Maarseveen, M.F.A.M. (2017) The characteristics of bike-sharing usage : case study in Zhongshan, China : open access. In: The international journal of transport development and integration, 1(2017)2, p. 245-255.

Zhang, Y., Thomas, T., Brussel, M.J.G. and van Maarseveen, M.F.A.M. (2016) Expanding bicycle-sharing systems : lessons learnt from an analysis of usage. In: PLoS ONE : open access, 11 (2016)12article no. e0168604, 25 p. 


\section{ITC Dissertation List}

https://www.itc.nl/Pub/research programme/Research-review-andoutput/PhD-Graduates 